\title{
Diagnostic Performance of a prototype Dual-Energy Chest Imaging System
}

by

Hany Mehdizadeh Kashani

A thesis submitted in conformity with the requirements for the degree of Master of Science

Institute of Medical Science

University of Toronto

(C) Copyright by Hany Mehdizadeh Kashani 2011 


\title{
Diagnostic Performance of a prototype Dual-Energy Chest Imaging System
}

\author{
Hany Mehdizadeh Kashani \\ Master of Science \\ Institute of Medical Science \\ University of Toronto
}

2011

\section{Abstract}

Purpose: To assess the performance of a Dual-Energy chest radiography system.

Methods: A cohort of 129 patients was recruited from population referred for CT guided biopsy of a lung lesion. Digital radiography (DR) and Dual Energy (DE) images were acquired. Receiver operating characteristic (ROC) tests were performed to evaluate performance of DE images compared to DR. Five chest radiologists scored images. Performance was analyzed for all cases pooled and sub groups based on gender, nodule size, density, location, and chest diameter.

Results: There was no significant difference between DE and DR for all cases $(p=0.61)$. There was a significant advantage for DE imaging of small nodules, and nodules located in right-upper lobe. $(\mathrm{p}=0.02$ and 0.01$)$ 
Conclusions: DE imaging demonstrated significant improvement in diagnosis of sub-centimeter lung nodules and lesions in the upper lung zones which are common characteristic of early stage lung malignancy. 
TO ALL THAT I DEARLY LOVE,<smiles>C[13CH2]</smiles> 


\section{Acknowledgments}

I once read a statement by Elwyn Brooks White to which I felt a deep connection:

"I arise in the morning torn between a desire to improve the World and a desire to enjoy the world. This makes it hard to plan the day."

And I wondered......, is there a way to enjoy and improve the World at the same time?

I have found my passion, in research, in the field of Medical Imaging. I believe that research plays an important role in improving and evolving technology. There is a keener awareness of potential health risks, even from small doses of radiation, than ever before, however, increased utilization of imaging modalities has ignited a grand revolution in detection and management of a wider spectrum of pathology. One of the most important aims for researchers in this field is to create techniques with less radiation exposure and better image quality, and that is what I have and will be involved with, something that makes me feel quite useful. Of course, I have two strong-minded men to thank for it, Dr. Narinder Paul and Dr. Jeff Siewerdsen and it has been an honor to be their pupil.

Jeff, I would like to thank you for trusting me with the DE project even though my engineering and physics experience were close to zero. It was very exciting to be in charge of the Dual Energy X-ray system, to optimize the images, perform clinical trials and to finally see the results of our experiment. I became familiar with Dual Energy innovative technology, troubleshooting, programming, Physics, Math and much more. A whole new World, so different from what I knew before and I absolutely fell in love with it. I also like to thank you for your support and guidance throughout this project that has given me the opportunity to win scientific prizes, publish and present our work in peer reviewed scientific journals and conferences.

Narinder, you have been my guiding light and my mentor since that fateful day in October of 2004 when I stepped through the doors of Toronto General Hospital. I was very 
confused but your nice attitude took away all my anxiety and gave me peace of mind and confidence. I was also quite amazed by all the advanced imaging modalities in your Department as well as your research projects involving low dose and very low dose CT. Suddenly it occurred to me! I would like to be a part of all this innovation, creativity and improvement. You are the reason that I could find my true path in life and I thank you for that. Thank you for teaching me, trusting me and motivating me. Your enthusiasm, discipline and eagerness to improve the world of Imaging have been quite encouraging for me. Your help and guidance made it possible for me to achieve a lot and I owe you the success in all my endeavours.

I want to acknowledge with deep gratitude the contributions of Care Stream Health Scientists and Engineers, including John Yorkston, and Rich Van Metter. I would also like to thank Drs. Leon Zelovitzky, Sidney Sussman, Demetris Patsios, Taebong Chung and all the Radiology fellows, Ute, Pryia, Andre, Yon-Mi and Diem who participated in the observer studies which were the main part of this thesis and led to very interesting and exciting results. I would also like to thank Dr. Masoom Haider and Dr. Tom Waddell who agreed to be part of my thesis committee and guide me throughout this project. I would like to express my gratitude towards the helpful and friendly Technologists, Nurses and the volunteer Patients at Toronto General Hospital who made this project happen. Last but not least I would like to thank my friends and colleagues Carlos Varon, Grace Gang, Nick Shkumat and Samuel Richard, the very popular "Dual Energy" team. Your positive attitudes and cooperation are very much appreciated. You helped me learn a lot and made my graduate experience enjoyable and memorable.

This project is supported in part by Carestream Health Inc and the National Institute of Health. 


\section{Table of Contents}

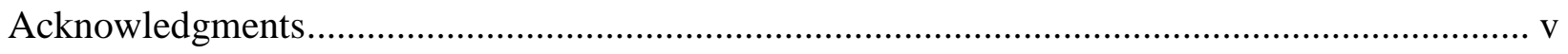

Table of Contents .......................................................................................................... vii

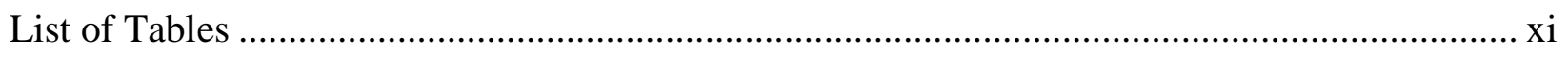

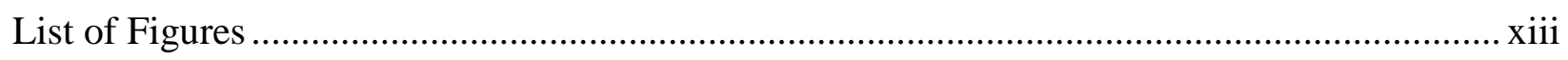

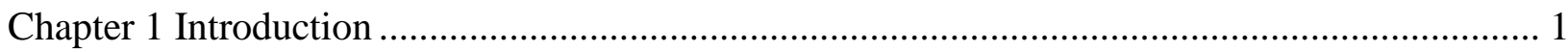

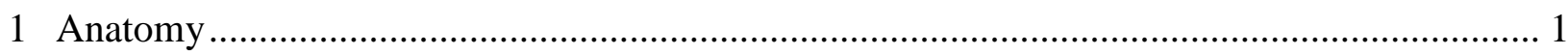

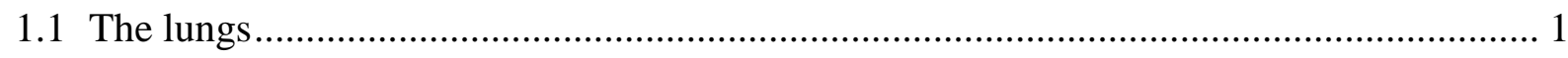

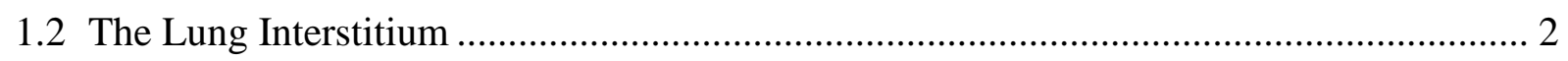

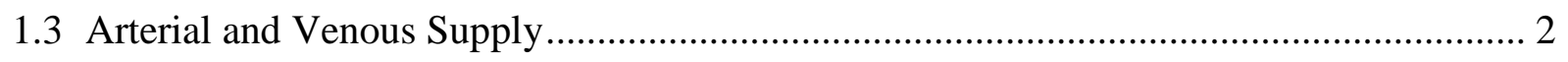

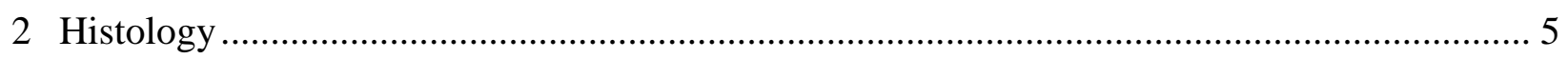

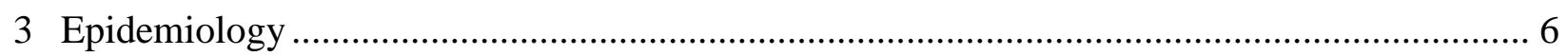

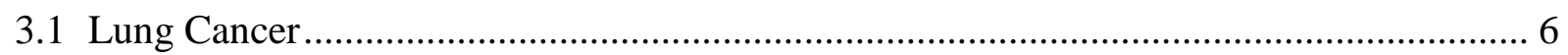

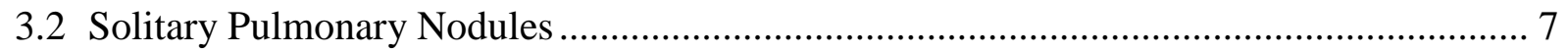

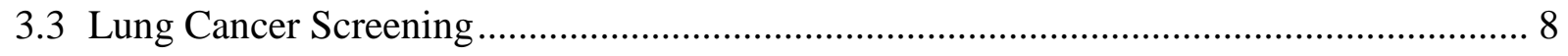

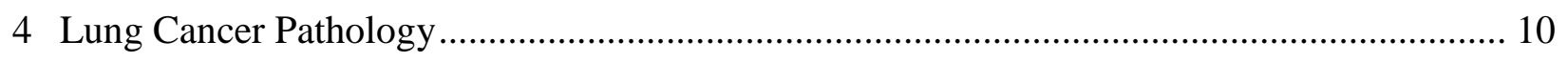

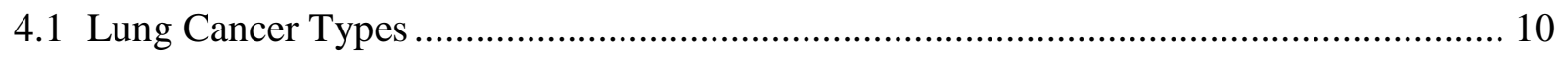

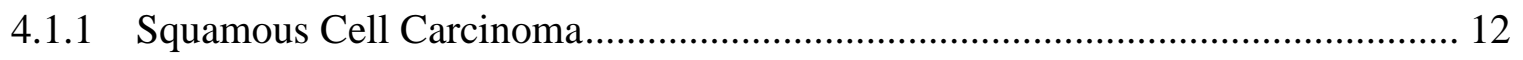

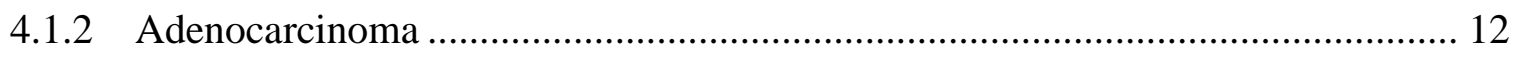

4.1.3 Small Cell Carcinoma .......................................................................... 13

4.1.4 Small Cell Carcinoma ............................................................................... 13

4.1.5 Large Cell Carcinoma ............................................................................. 14

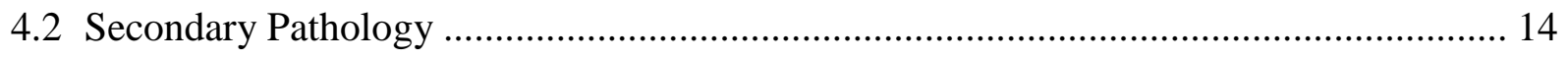

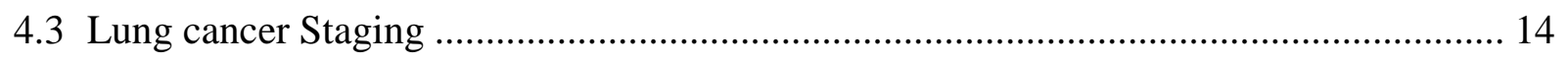

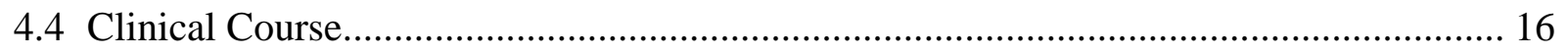




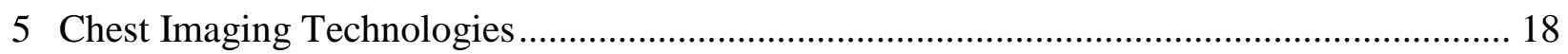

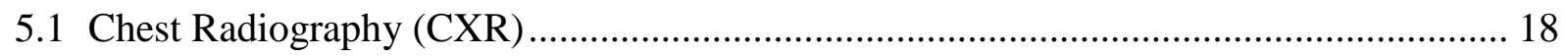

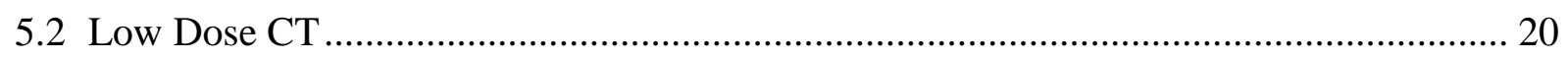

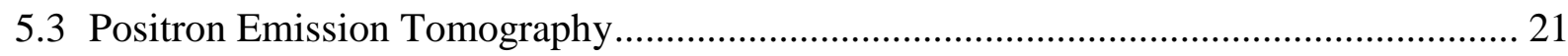

5.4 Dual Energy Chest Imaging ..................................................................................... 23

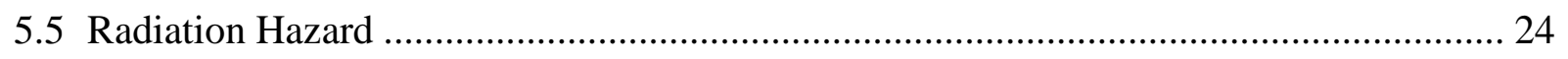

Chapter 2 Dual Energy Prototype and Patient Cohort ................................................................... 26

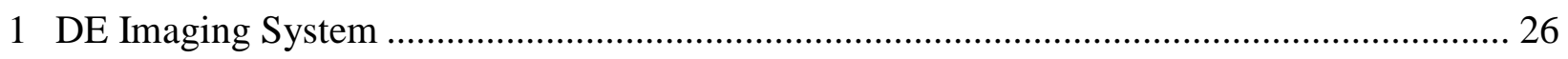

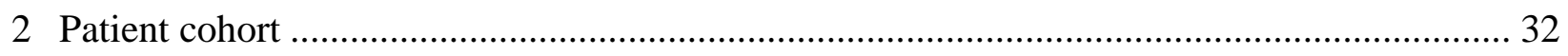

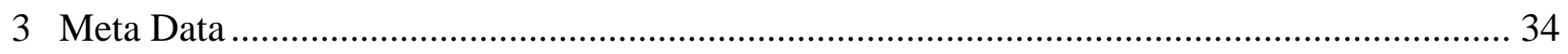

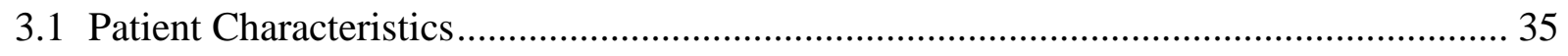

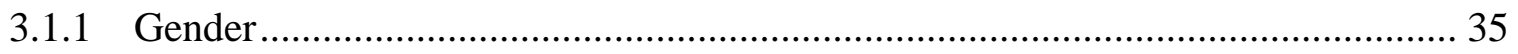

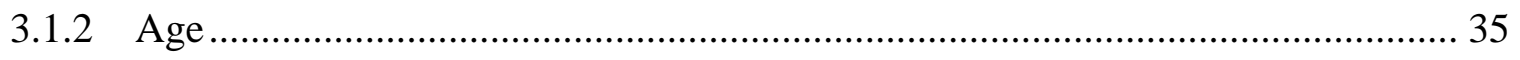

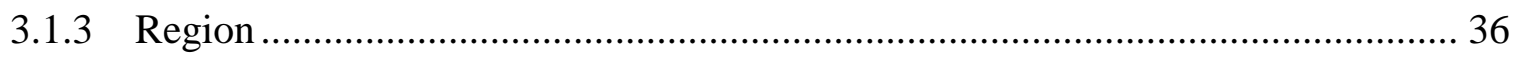

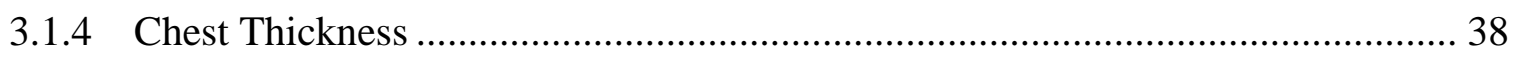

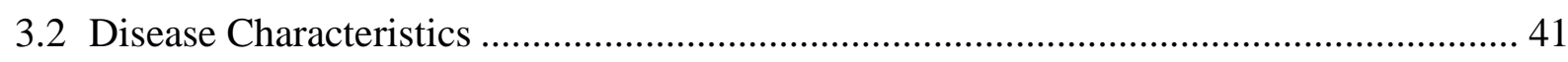

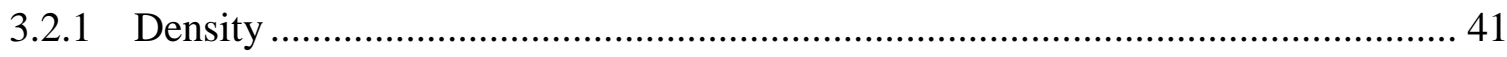

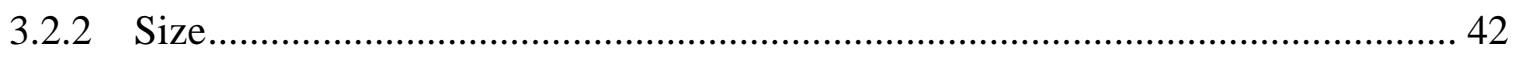

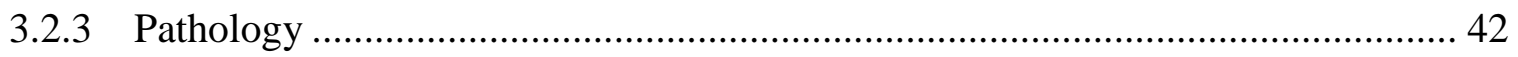

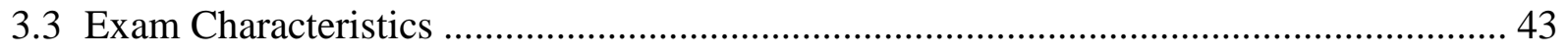

3.3.1 Repeat Breath Hold ................................................................................... 43

3.3.2 Time between the Exposures ........................................................................ 44

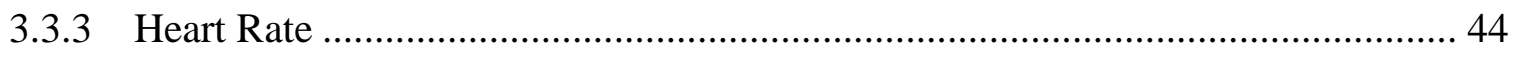

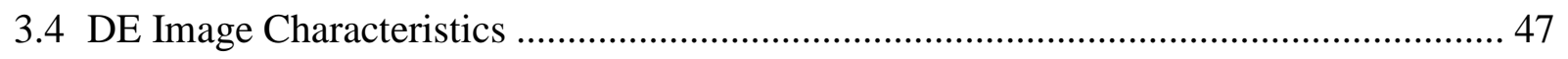

3.4.1 Tissue Cancellation Parameters: Ws, Wb, Wc, Wn.............................................. 47

3.5 Multi Scale Multi Resolution (MSMR) Registration Algorithm........................................ 49 


\subsubsection{Mean Vector displacement (MVD) and Standard Deviation Displacement}

(SDD)

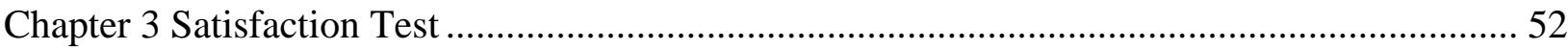

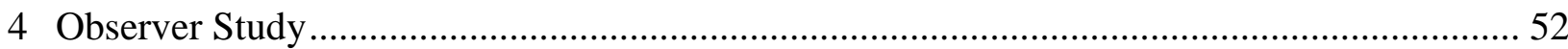

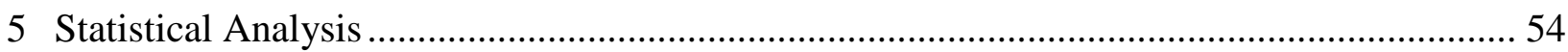

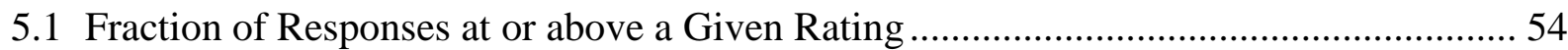

5.2 Statistical Significance: P-value from Wilcoxon Signed Rank Test ……………............. 55

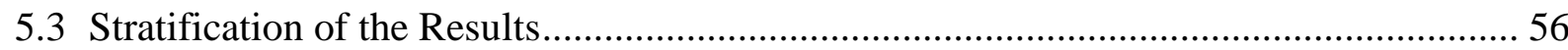

6 Result

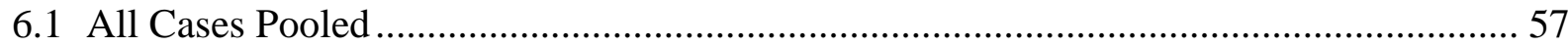

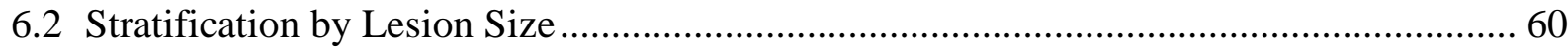

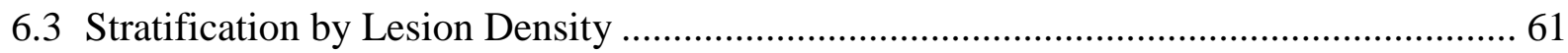

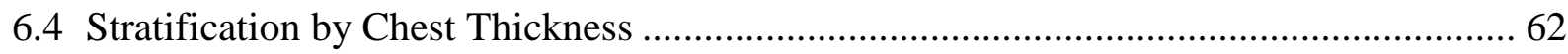

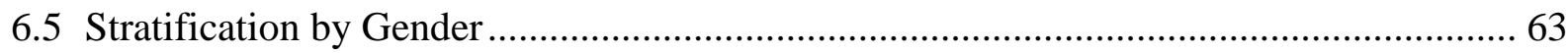

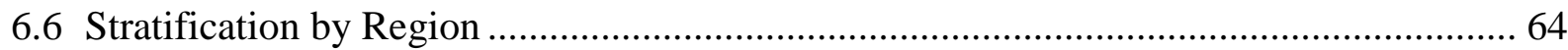

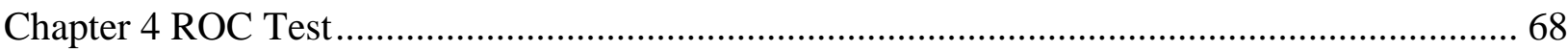

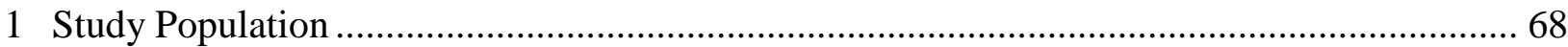

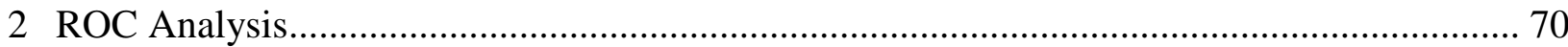

2.1 ROC Curves and Area under the Curve (AUC) ............................................................. 70

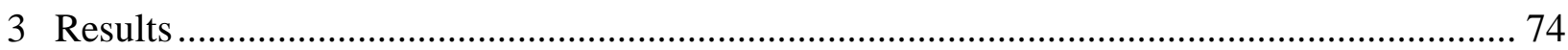

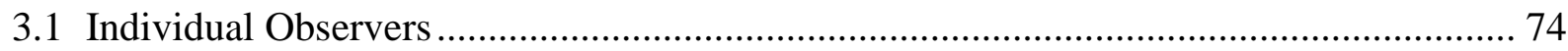

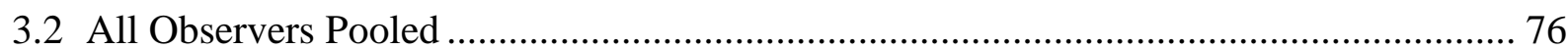

3.3 Diagnostic Accuracy and Clinical Significance ……………………............................ 79

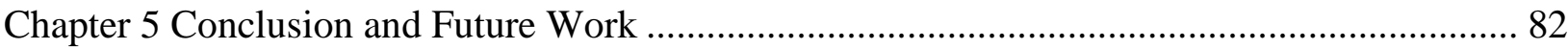

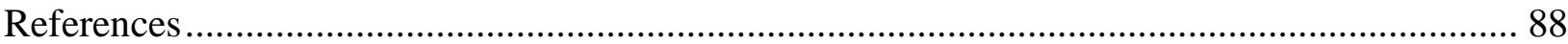

63. Rockette HE, Gur D. "Selection of a rating scale in receiver operating characteristic studies: some remaining issues" Acad Radiol. 2008; 15(2):245-8. 
Copyright Acknowledgements.. 


\section{List of Tables}

Table 1.1 TMN staging. The TNM staging system takes into account the degree of spread of the primary tumour, represented by $\mathrm{T}$; the extent of regional lymph node involvement, represented by $\mathrm{N}$; and the presence or absence of distant metastases, represented by $\mathrm{M}$.

Table 1.2 Subtypes of lung cancer based on therapeutic and prognostic implication.

Table 2.1 Optimal technique chart for Dual energy projections based on patient thickness.

Table 2.2 Optimal technique chart for digital radiograph projection based on patient thickness.

Table 3.1 Satisfaction rating scale. This table was displayed to observers on a second monitor during all tests as shown in table 3.1.

Table 3.2 Summary of Cases and Stratifications of Data. The $P$ values relate to the statistical significance in the difference between satisfaction with dual-energy and digital radiographic images, regarded as significant for $\mathrm{P}<.01$.

Table 4.1 Five-point Rating Scale for Receiver-Operating Characteristic Studies.

Table 4.2 Summary of cases overall and within each post-hoc stratification.

Table 4.3 The area under the curve (AUC) for each observer is shown, found to be superior for dual energy (DE) imaging in three of five observers but not to a significant extent (two-tailed $P$ value based on AUC).

Table 4.4 The total number of readings for each modality was 1290 (equal to 258 cases - 5 radiologists), with 790 actually positive and 500 actually negative cases. Results were analyzed in terms of the AUC for dual energy (DE) imaging and digital radiography (DR) (area under the curve [AUC] DE and AUC DR, respectively).Statistical significance in the observed differences were analyzed in terms of the P value associated with the entire AUC. Statistically significant results are highlighted in bold text.

Table 4.5 Accuracy, Definitiveness, and Clinical Significance, Dual energy (DE) imaging was more accurate than digital radiography (DR) in identifying actually positive cases but less 
accurate in ruling out actually negative cases. DE imaging was more definitive in properly identifying both actually positive and actually negative cases. 


\section{List of Figures}

Figure 1.1 Illustration of lungs and bronchial tree. Lungs are located just above the first rib down to the diaphragm. Modified from Healthuse.com.

Figure 1.2 (a) Pulmonary and bronchial circulation of the respiratory system. Pulmonary arteries carry the deoxygenated blood from the right side of the heart and to the lung. Pulmonary veins carry oxygenated blood to the left side of the heart. (b) The bronchial arteries supply bronchi and the non-respiratory part of the lungs. They drain to the azygos.

Figure 1.3 Structure of alveolus. The alveoli epithelium contains two different cell types, type I and type II. Type I cells are responsible for gas exchange and type II secrete the surfactant coating to reduce surface tension. Modified fromherkules.oulu.fi/isbn9514270584/html/c273.html

Figure 1.4 The anteroposterior (a) and lateral (b) views of male chest x-ray. Main anatomical regions are labeled.

Figure 1.5 Comparison of the anatomical detail displayed on a frontal chest radiograph and an axial projection from a thoracic CT study taken at the level of the main pulmonary trunk.

Figure 1.6 Projection images from a FDG PET scan (lower row) demonstrate intense uptake within a right lower lobe tumour. The top row shows the corresponding CT images (lung reconstruction (left) and mediastinal reconstruction, (right)). The tumour is seen at the same location. Modified from www.chestjournal.org/.../124/3/893/F1.expansion

Figure 2.1 Illustration of dual-energy (DE) image processing, registration (Reg), decomposition (Decomp) and display. Processing steps include offset, gain, and defect correction; deformable registration; weighted log-subtraction decomposition; and the transformation of pixel values to log-exposure space for display. The photograph on the right shows the experimental setup for observer studies, with images displayed on the left and the rating scale on the right. Bone: bone decomposition; Comp: composite equivalent radiograph; HE: high-energy projection; LE: lowenergy projection; Soft: soft tissue decomposition. 
Figure 2.2 Example dual energy (DE) and digital radiography (DR) images. DE components include: (A) the soft-tissue image, $(B)$ the bone image, and $(C)$ the composite image. The corresponding DR image is shown in (D). The location of a $2.4 \mathrm{~cm}$ nodule in the left upper lobe is marked by an arrow.

Figure 2.3 Patient accrual into five different research arms to evaluate variable DE parameters. Figure 2.4 A histogram showing the number of cases in each age group. The majority are between 55 and 75 years of age.

Figure 2.5 A histogram showing the anatomical location of the lung nodule undergoing biopsy. As shown in the histogram, the most frequent location was the right upper lobe. Anatomical lobes of lungs: 1. Right Upper Lobe=RUL, 2. Right Middle Lobe=RML, 3. Right Lower Lobe=RLL, 4. Left Upper Lobe=LUL, 5. Left Middle Lobe= Lingula, 6. Left lower Lobe=LLL, $0=$ Mediastinum.

Figure 2.6 Histograms of chest measurements versus frequency in total patient and in each gender individually. The majority of cases have the anteroposterior diameter of the average size patient, being $24-26 \mathrm{~cm}$.

Figure 2.7 Regression analysis for measuring the anteroposterior diameter on the CT axial projection and by using a manual caliper. R-squared is quite high, showing the strong relationship between the two variables.

Figure 2.8 A histogram showing the number of cases in each density group.

Figure 2.9 A histogram showing the number of cases in each nodule size group.

Figure 2.10 (a, b) Illustration of lung nodule pathology. a) The most common pathology was non-small cell carcinoma and b) the most common type of non-small cell carcinoma was adenocarcinoma.

Figure 2.11 Projections acquisitions frequency for different time delay intervals. As observed in the histogram above, most of the studies were performed with an average delay of 6-9 seconds. 
Figure 2.12 HR variation during high- and low-energy projections. As shown here, most of the patients had a HR of about $60-80$ (beat/sec) during projections. But there is a shift towards a higher rate during the high-energy projection, the second acquisition, due to compensatory tachycardia in order to offset the reduction in cardiac venous return with breath holding.

Figure 2.13 Regression analysis. The coefficient of determination for heart rate during low- and high-energy projections. The HRs are highly correlated $(\mathrm{R} 2=0.69)$.

Figure 2.14 (a, b, c, d) Frequency of cases in different Weighting factor (W) ranges for different decompositions.

Figure 2.15 (a, b) Histograms showing frequency of cases in different ranges of MVD and SD of MVD.

Figure 2.16 (a, b) Histograms showing frequency of cases in different ranges of Max-MVD and SD of deformity.

Figure 3.1 Diagnostic satisfaction in dual-energy (DE) and digital radiographic (DR) image readings (all cases pooled): (a) percentage of image pairs for which the DE image was rated superior, equal, or inferior to the DR image; (b) percentage of image pairs for which the DE image was rated superior, equal, or inferior to the DR image as agreed by three or more observers; (c) fraction of observer responses at or above a given rating score. The error bars represent 95\% confidence intervals.

Figure 3.2 Diagnostic satisfaction in dual-energy (DE) and digital radiographic (DR) image readings stratified by lesion size. The curves show the fraction of responses at or above a given rating score for (a) lesion size $\leq 3 \mathrm{~cm}$ and (b) lesion size $>3 \mathrm{~cm}$. The error bars reflect $95 \%$ confidence intervals.

Figure 3.3 Diagnostic satisfaction in dual-energy (DE) and digital radiographic (DR) image readings stratified by lesion density. The curves show the fraction of responses at or above a given rating score for (a) nonsolid lesions (<20 Hounsfield units [HU]) and (b) solid lesions (>20 HU). The error bars reflect 95\% confidence intervals. 
Figure 3.4 Diagnostic satisfaction in dual-energy (DE) and digital radiographic (DR) image readings stratified by chest thickness. The curves show the fraction of responses at or above a given rating score for (a) thickness $<26 \mathrm{~cm}$ and (b) thickness $>26 \mathrm{~cm}$. The error bars reflect $95 \%$ confidence intervals.

Figure 3.5 Diagnostic satisfaction in dual-energy (DE) and digital radiographic (DR) image readings stratified by patient gender. The curves show the fraction of responses at or above a given rating score for (a) men and (b) women. The error bars reflect $95 \%$ confidence intervals.

Figure 3.6 Diagnostic satisfaction in dual-energy (DE) and digital radiographic (DR) image readings stratified by lesion location. The curves show the fraction of responses at or above a given rating score for lesion located in (a) right upper, (b) left upper, (c) right middle, (d) left middle, (e) right lower, (f) left lower, and (g) mediastinal regions. The error bars reflect $95 \%$ confidence intervals. For lower satisfaction scores $(\leq 5)$ reflecting lower satisfaction, there is no clear pattern however for higher satisfaction scores $(>5)$, DE images demonstrate an advantage over DR.

Figure 3.7 Illustration of (a) dual-energy soft tissue, (b) dual-energy bone, and (c) digital radiographic images for a case exhibiting a small, solid nodule $(0.8 \mathrm{~cm}, 58.4$ Hounsfield units, benign lymphoid tissue), marked by the arrows. The bone image exhibits some residual soft tissue attributable to incomplete cancellation as well as motion artifact, which is more obvious in this case because of the cardiac motion artifact.

Figure 3.8 Illustration of (a) dual-energy soft tissue, (b) dual-energy bone, and (c) digital radiographic images for a case exhibiting a nodule $(2 \mathrm{~cm}, 67.5$ Hounsfield units, adenocarcinoma) in the right upper lobe, marked by the arrows

Figure 3.9 Illustration of (a) dual-energy soft tissue, (b) dual-energy bone, and (c) digital radiographic images for a case exhibiting a nodule $(2.8 \mathrm{~cm}, 35.1$ Hounsfield units, adenocarcinoma) in the left apex, marked by the arrows. 
Figure 4.1 The distribution of rating scores among all observers for Dual energy (DE) and DR. As observed on the graphs, the distribution is non parametric and therefore non parametric ROC was applied.

Figure 4.2 Receiver-operating characteristic (ROC) curves for five observers. Dual energy (DE) outperformed digital radiography (DR) for three of five readers ( $a, b$, and e), although the difference in performance was not statistically significant $(P>.05)$.

Figure 4.3 a, b. Receiver-operating characteristic (ROC) curves for five nodules smaller than 1 mm (a) and nodules located in the Right Upper Lobe. (b) DE outperformed DR in these two categories $(P<.05)$

Figure 4.4 Example of dual energy (DE) and digital radiography (DR) images for a small nodule. The location of a $0.9 \mathrm{~cm}$ nodule in the right upper lobe with density of $28.5 \mathrm{HU}$ is marked by an arrow.

Figure 4.5 Example of dual energy (DE) and digital radiography (DR) images for a nodule located in right upper lobe. The location of a $3-\mathrm{cm}$ nodule with density of $42.9 \mathrm{HU}$ is marked by an arrow. 


\section{Chapter 1 \\ Introduction}

\section{Anatomy}

\subsection{The lungs}

There are two lungs located on each side of the thoracic cavity and covered by the rib cage and separated by mediastinal structures which include the heart. The superior boundary of the lungs is located just above the first rib, inferiorly the lungs rest on the diaphragm which separates the chest from the abdominal cavity. (Fig 1.1) The lungs are covered by two thin membranes: the parietal and visceral pleura. The visceral pleura covers the lungs directly and the parietal pleura covers the inside of the chest wall. The hilum of each lung is the point of attachment for the root of the lung. It contains the bronchi, pulmonary, bronchial and lymphatic vessels, and nerves.

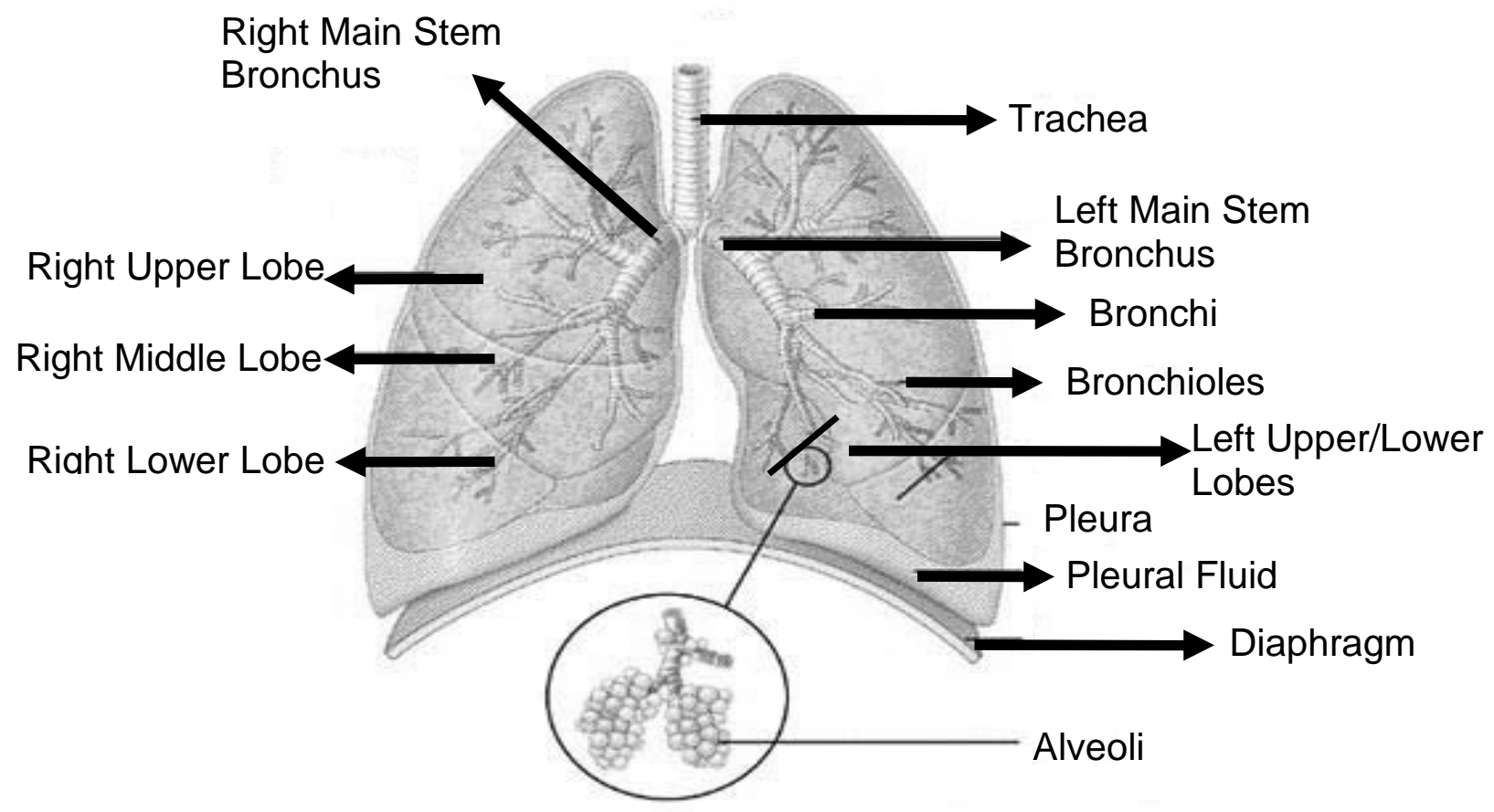


Figure 1.1 Illustration of lungs and bronchial tree. Lungs are located just above the first rib down to the diaphragm. Modified from Healthuse.com.

The right lung is divided by the oblique and horizontal fissure into three lobes: upper, middle and lower. The left lung contains only the oblique fissure that divides the lung into upper and lower lobes. The lingua of the upper lobe corresponds to the middle lobe of the right lung. Each lobe consists of multiple segments and each segment has multiple lobules.

\subsection{The Lung Interstitium}

The lung interstitium is a network of connective tissue fibers with several components that support the lung. These components include: peribronchovascular interstitium, centrilobular interstitium, interlobular interstitium, interlobular septa, and sub-pleural interstitium.

The peribronchovascular interstitium is a system of fibers that surrounds bronchi and pulmonary arteries to form a strong supportive sheath of connective tissue. The subpleural interstitium invests the lungs in a fibrous sac and is located deep to the visceral pleura. From this fibrous sac, connective tissue septa penetrate into the lung parenchyma.

The interlobular septum is part of these septa. The intralobular interstitium consists of thin fibers that form a mesh in the alveolar walls, connecting the centrilobular interstitium located within the centre of lobules to the interlobular septa and subpleural interstitium in the lobular periphery. These fibers integrate to form a protective fibrous skeleton for the lungs.

\subsection{Arterial and Venous Supply}

The right and left pulmonary arteries arise from the pulmonary trunk. The pulmonary arteries deliver deoxygenated blood to the lungs from the right side of the heart (Fig1.3. a). The bronchial arteries arise from the descending thoracic aorta to supply the bronchi and the non- 
respiratory portion of the lung (Fig1.3. b). There are four pulmonary veins: superior right and left and inferior right and left. The pulmonary veins carry oxygenated blood to the left atrium of the heart (Fig1.3. a). The bronchial veins drain to the azygos system. They share drainage from the bronchi with the pulmonary veins. (Fig1.3. b)

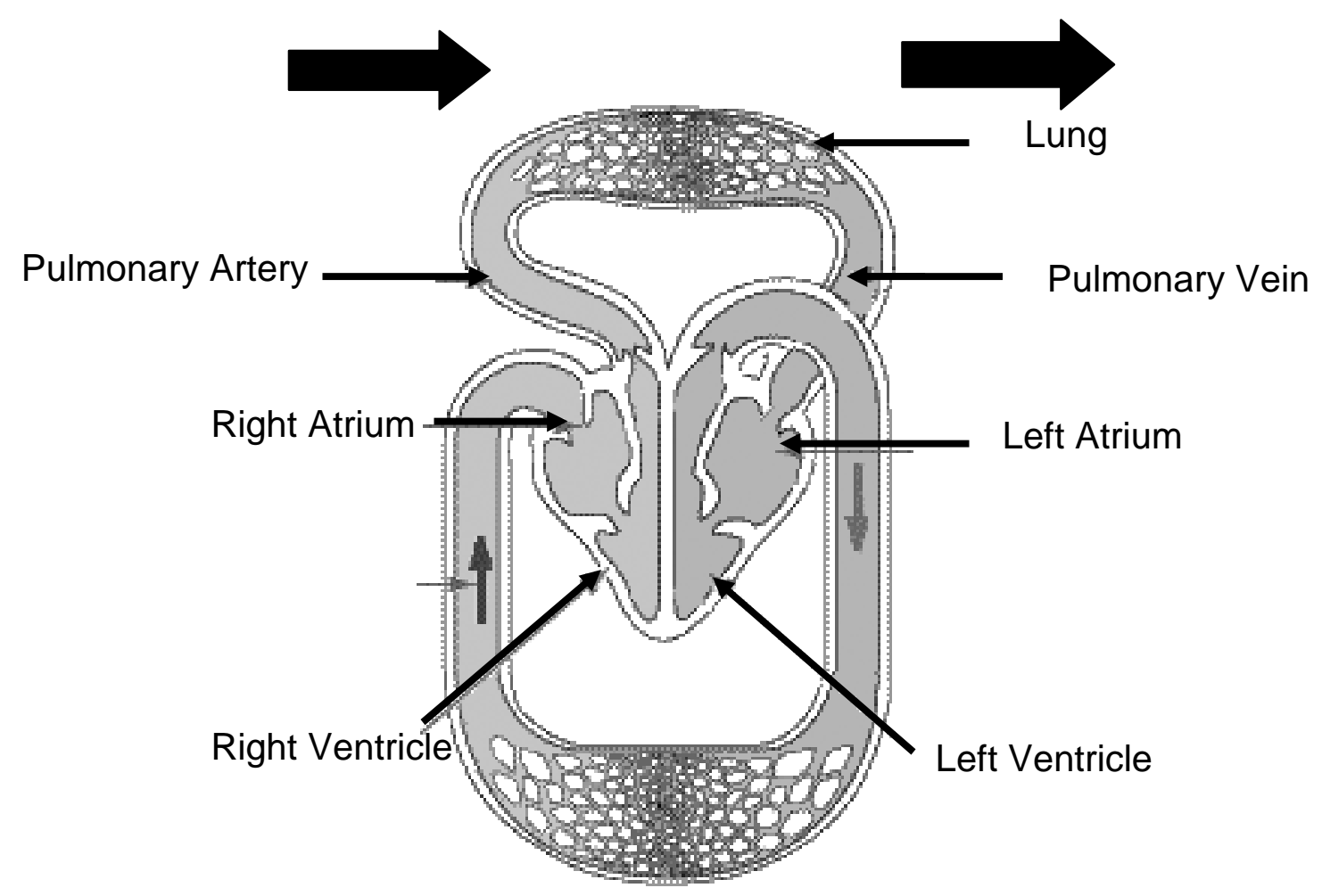

(a) Pulmonary circulation.

Modified from http://webschoolsolutions.com/patts/systems/lungs.htm 


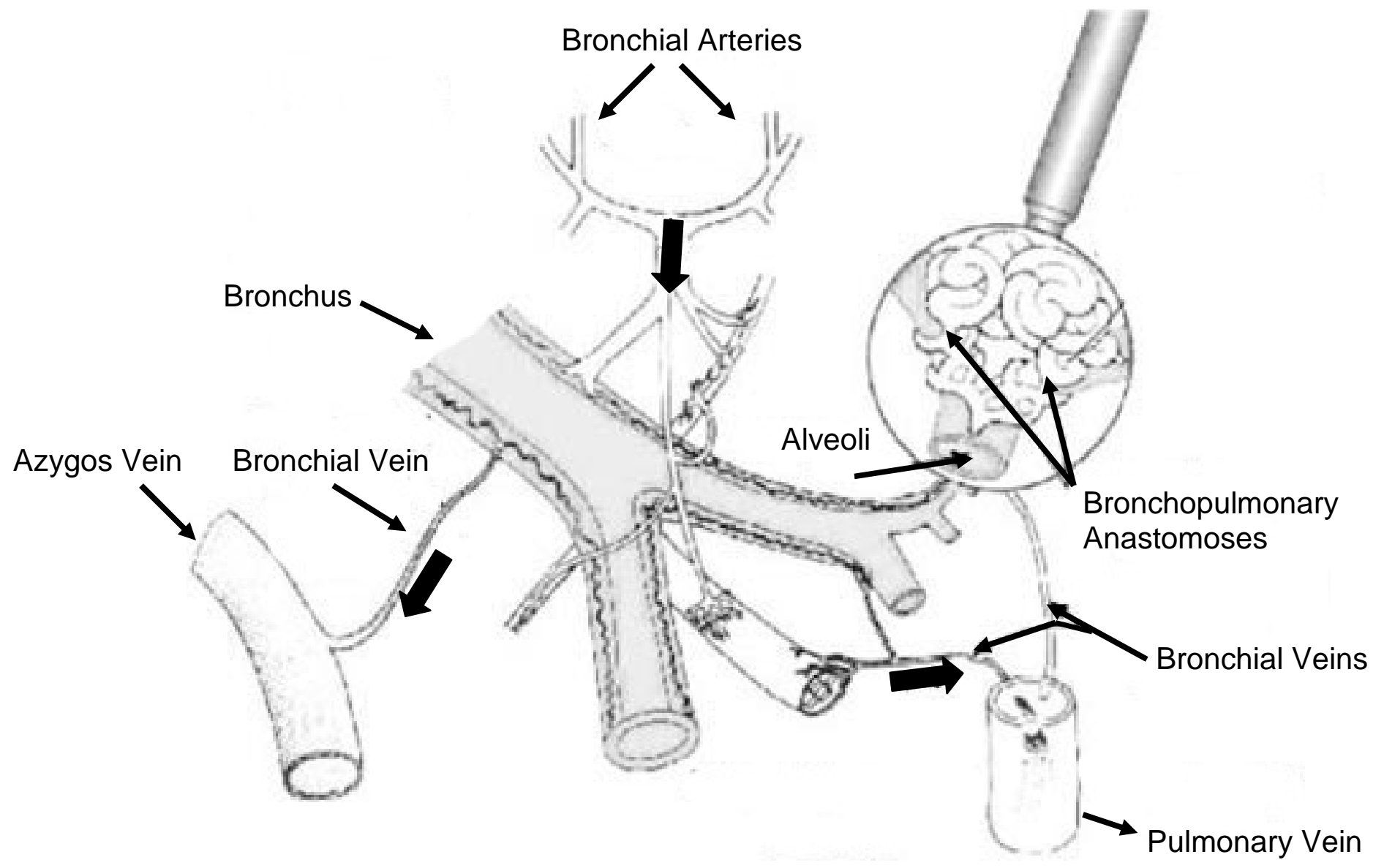

(b) Bronchial circulation.

Modified from http://webschoolsolutions.com/patts/systems/lungs.htm

Figure 1.2 (a, b) Pulmonary and bronchial circulation of the respiratory system. Pulmonary arteries carry the deoxygenated blood from the right side of the heart and to the lung. Pulmonary veins carry oxygenated blood to the left side of the heart. (a) The bronchial arteries supply bronchi and the non-respiratory part of the lungs. They drain to the azygos. (b) 


\section{Histology}

The respiratory system is divided into an air conducting portion, including the nasal cavity, pharynx, larynx, trachea, bronchi and bronchioles, that carries the gases during inspiration and expiration, and a respiratory portion, including alveoli, that provides for gas exchange between air and blood. The trachea leads to the main terminal and respiratory bronchioles. In the transition from the trachea to the respiratory bronchioles, the epithelium changes from pseudostratified ciliated columnar cells to simple columnar cells. (4)

Terminal bronchioles lead to respiratory bronchioles that contain alveoli and branches to form two to three alveolar ducts, which are long sinuous tubes that often terminate in alveolar sacs. Alveolar sacs are spaces formed by two or more conjoined alveoli. They are lined by simple squamous alveolar epithelia. Alveoli are the terminal thin-walled sacs of the respiratory tree that are responsible for gas exchange. There are approximately 300 million alveoli per lung, each of which is 20 to $30 \mathrm{~mm}$ in diameter. (5)

The alveoli epithelium contains two cell types. Type I cells cover almost the entire alveolar luminal surface and provide a thin surface for gas exchange. Type II cells are cuboidal-like cells that sit on the basal lamina of the epithelium and contain membrane-bound granules of phospholipids and protein. These contents are secreted onto the alveolar surface to provide a coating of surfactant that reduces alveolar surface tension. (6) (Fig 1.4) 


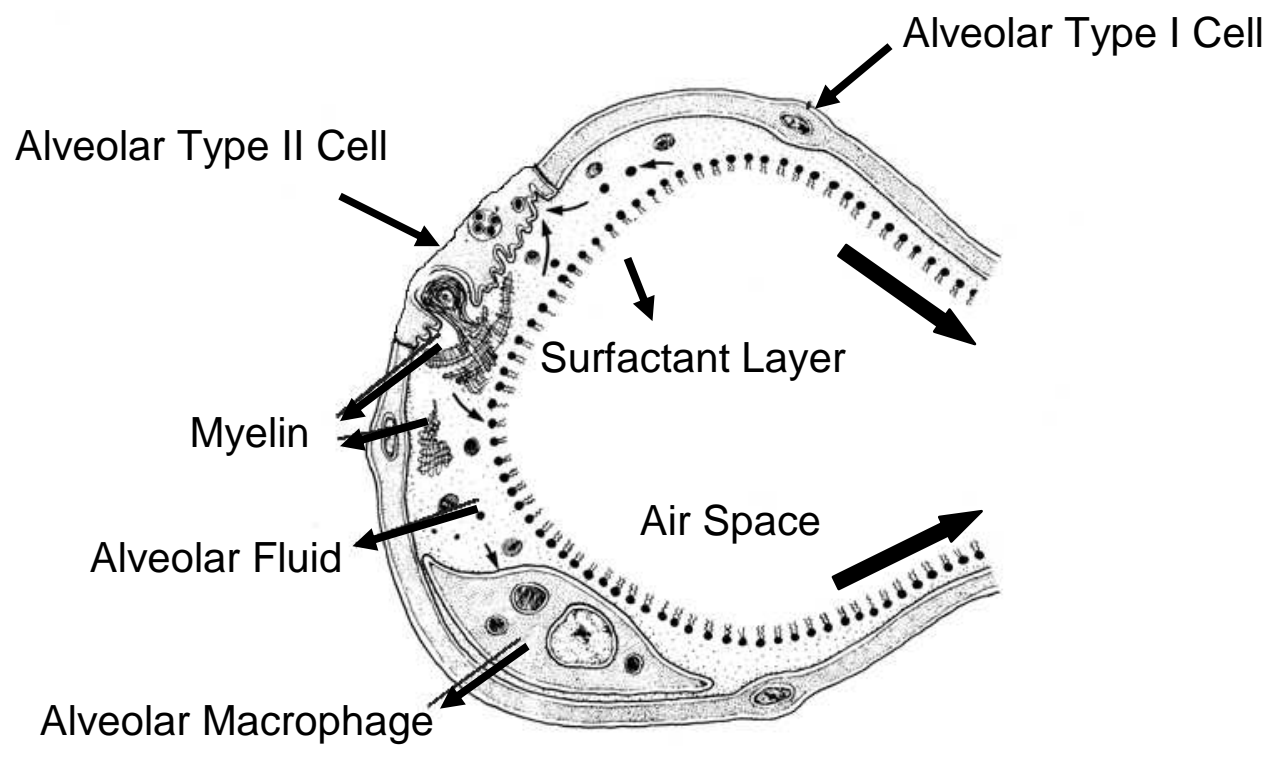

Figure 1.3 Structure of alveolus. The alveoli epithelium contains two different cell types, type I and type II. Type I cells are responsible for gas exchange and type II secrete the surfactant coating to reduce surface tension. Modified from herkules.oulu.fi/isbn9514270584/html/c273.html

\section{Epidemiology}

\subsection{Lung Cancer}

Cancer is the leading cause of death worldwide, accounting for 7.4 million deaths or around $13 \%$ of all deaths in 2004. (7) Among the different types of cancer, lung cancer remains the leading cause of cancer-related deaths, killing 1.3 million people each year. (7) Lung cancer is the second-most frequently diagnosed cancer in both men and women; after prostate and breast cancers which are the most frequent in men and women, respectively. (8) In Canada, lung cancer continues to be the leading cause of cancer mortality with an estimated 9,400 deaths occurring among women and an estimated 11,200 deaths among men in 2009. (9) In 2010, an estimated 24,200 Canadians will be diagnosed with lung cancer and 20,600 will die of it. Lung cancer remains the leading cause of cancer death for both men and women. (10) 
Lung cancer usually presents in patients aged between 40 and 70 years, with the peak incidence in the sixth decade. (11) There is evidence that cigarette smoking is a major causal factor. Clinical studies have observed the changes in the epithelium lining of the respiratory tract in smokers and non-smokers. (12) Loss of ciliated cells, basal cell hyperplasia, squamous metaplasia and atypia of cells are the changes that have been noticed among smokers. (13) Also, the risk of developing lung cancer is increased by industrial exposure to carcinogens such as radiation, uranium, asbestos, nickel, chromates, coal, mustard gas, arsenic, beryllium, and iron. (14) Genetic predisposition is also known to be associated with bronchogenic carcinoma. Approximately $10 \%$ of these cancers occur in non-smokers; although it should be mentioned that bronchogenic carcinoma in non-smokers is usually of a different subgroup than in smokers' type. Occasionally, lung cancers arise in scar tissue and are termed scar cancers. Histologically, these are usually of an adenocarcinoma subtype. These scars are usually due to old infarcts, wounds, foreign bodies or granulomatous infections such as tuberculosis.

\subsection{Solitary Pulmonary Nodules}

A solitary pulmonary nodule is defined as a discrete lesion $<3 \mathrm{~cm}$ in diameter that is surrounded by lung parenchyma (i.e., does not touch the hilum, mediastinum, or pleura). Solitary pulmonary nodules are most often detected incidentally when a chest x-ray is taken for other reasons. Every pulmonary nodule could be an early stage of a lung cancer; therefore it is vital to distinguish a benign nodule from a cancerous one. Solitary pulmonary nodules have many causes, including inflammatory lesions, granulomas and infections. (15) Patient age and risk factors play an important role in assessing the likelihood of malignancy. The primary goal in evaluation of any nodule should be to differentiate benignity from malignancy and active infection. Although a thorough history and physical exam is the first step in approaching a patient with a pulmonary 
nodule, the first imaging test that is usually performed is a chest x-ray followed by a thoracic CT scan if necessary. There are some important radiographic characteristics that help to distinguish a benign from a malignant nodule:

1) Growth rate, which is determined by comparing the current images with previous ones. No enlargement in more than 2 years suggests a benign etiology. Tumors with volume doubling times from 21 to 400 days have a higher probability of being malignant.

2) Presence of calcification if it is central, concentric or in a popcorn configuration suggests benign disease.

3) Margins that are spiculated or irregular are more indicative of malignancy.

4) Diameter less than $1.5 \mathrm{~cm}$ strongly suggests a benign etiology and more than $5.3 \mathrm{~cm}$ strongly suggests cancer although there are some exceptions, which include lung abscess, Wegener granulomatosis, and hydatid cysts.

Since the clinical stage at the time of diagnosis is the major factor in survival rate after therapy, there has been a lot of interest in early lung cancer screening lately using the low dose CT which is a highly sensitive modality for detection of lung nodules.

\subsection{Lung Cancer Screening}

As advances in computed tomography allow detection of very small pulmonary nodule at lower radiation doses low dose computed tomography seems to play a very important role in early detection of lung cancer. The International Early Lung Cancer Action Project (I-ELCAP) (16) with over 35,000 subjects enrolled is currently one of the most prominent studies of base line screening for lung cancer. It involves a baseline and an annual repeat CT following the I-ELCAP 
regimen of screening. New nodules that are identified are investigated within a specified protocol incorporating frequent CT scans, positron emission tomography scans, antibiotics or lung biopsy. Patients found to have lung cancer undergo surgical resection.

According to IELCAP data, the 10 year survival rate for these patients is $88-92 \%$. Although these results seem very favorable and encouraging, there are concerns: according to the Mayo Clinic experience (17) only $1.3 \%$ of the detected nodules are malignant therefore $\sim 99 \%$ of the detected nodules include other abnormalities which had unnecessary further work up. Although not all of these patients had lung biopsies performed, all of them were subjected to high levels of anxiety while waiting for the result of their tests. (18) A second methodological issue relates to the inherent biases on the observed results: selection bias, lead-time bias, length bias, and over diagnosis bias. Selection bias is the error in choosing the subjects to take part in a study. Ideally, they should be very similar to one another and to the larger population from which they are drawn (for example, all individuals with the same disease or condition). If there are important differences, the results of the study may not be valid. It can affect the mortality as well as other measures of outcome, but in a randomized trial, it is eliminated. All the observed results from the lung cancer screening studies, except mortality were subject to lead-time bias, length bias, and over diagnosis bias. (19) None of these studies demonstrated any significant improvements in the mortality rate for the screened population.

Lead time bias is one screening feature that could explain increased incidence, better survival and no decrease in mortality rate. If a screen detected cancer results in death exactly at the same time as it would have without screening, the only influence of screening was an earlier detection of the same fatal cancer. It can also explain "increased incidence" by leading to excess of cases in the screening population due to early detection. Over diagnosis bias happens when screening 
detects lesions that are not clinically important and would not make any difference to the patient's life span. Length bias can be explained by the fact that most indolent tumors have a long pre clinical phase and therefore are more likely to be diagnosed during screening; whereas more aggressive tumors progress and present relatively quickly and therefore are less likely to be detected by screening. This could explain the observed increased survival in screening populations compared to the general population as most of the malignancies detected during screening are indolent, slow growing tumors.

The National Lung Screening Trial (NLST) is a multicenter study, and the largest randomized trial of lung cancer screening to date and it compares low dose helical computed tomography with chest radiography in an at-risk population. This study has enrolled 53,456 participants over a period of almost two years from September 2002 to April 2004. Participants had one baseline and two annual screenings, using either chest radiography or low dose thoracic CT. The end point of this trial was to assess the utility of low dose CT on lung cancer mortality. The five years survival rate approached $70 \%$ in patients with surgical resection of stage IA disease but in patients with metastatic disease the survival rate was less than 5\%. (20). Recently, the National Cancer Institute (NCI) issued a press release of trial results that demonstrate a significant mortality benefit from screening with low-dose CT. There are twenty percent fewer lung cancer deaths among those who were screened with low dose helical CT as compared with chest X-ray.

\section{$4 \quad$ Lung Cancer Pathology}

\subsection{Lung Cancer Types}

A wide spectrum of benign and malignant tumours can occur in the lungs, and bronchogenic carcinoma is the most common with an incidence rate of $90-95 \%$ of all lung tumours. (22) It is 
followed in prevalence by bronchial carcinoid (5\%), mesenchymal tumours and other miscellaneous neoplasms $(2-5 \%)$. (23) This means that any lung primary malignancy can be assumed to be bronchogenic carcinoma until proven otherwise. Bronchogenic refers to the origin in the bronchial and sometimes bronchiolar epithelium. It usually arises in and around the hilus of the lung. About $75 \%$ of the lesions take origin from the lower trachea, first, second and third order bronchi. A small percentage of carcinomas are located in the lung periphery. Histologically, bronchogenic carcinoma is divided into four different subtypes:

1) Squamous cell carcinoma, 25-40\%, 2) Adenocarcinoma, 25-40\%, 3) Small cell carcinoma, 20-25\%, 4) Large cell carcinoma, 10-15\%.

Although each lesion usually consists of one specific cell type, there might be a mixture of histological patterns in a single lesion. It is important to know that squamous cell carcinoma has the strongest relationship to smoking among the different histological types. There is also another classification based on the lesion response to therapy, which includes:

A) Small cell carcinomas with high initial response to chemotherapy

B) Non-small cell carcinoma which is less responsive to chemotherapy.

In its developmental pathway, bronchogenic carcinoma begins as an area of in situ cytologic atypia. Then, over an unknown interval of time, it yields a small area of thickening of the bronchial mucosa. This small area usually grows to about $1 \mathrm{~cm}$. It usually appears as an irregular warty area that erodes the lining epithelium. The lesion may then follow one of the following paths:

A) It may fungate into the bronchial lumen to produce an intraluminal mass. 
B) It may rapidly penetrate the wall of the bronchus to infiltrate along the peribronchial tissue into the adjacent region of the carina or mediastinum. In his fashion, the tumour may even extend to about the pericardium or to the pleural surface and then within the pleural cavity.

C) It may also grow along a broad front to produce a cauliflower intraparenchymal mass that appears to push lung substance ahead of it.

The lesion is usually gray-white and firm to hard and may contain focal areas of hemorrhage and necrosis with cavitations, especially when the tumours are bulky. Almost $50 \%$ of cases spread to the tracheal, bronchial and mediastinal nodes. Distant metastasis usually spread widely through hematogenous or lymphatic pathways. Occasionally, the first manifestation of the underlying carcinoma is due to metastatatic disease. The most commonly involved organs include the adrenals (50\%), liver (30\%), brain (20\%), bone (20\%), and the kidneys (15\%).

\subsubsection{Squamous Cell Carcinoma}

Squamous cell carcinoma has the strongest relationship to cigarette smoking and is more common in men than women. This tumour arises in the larger, more central bronchi, tends to spread locally and metastasizes later than the other cell types. A squamous cell lung tumour doubles its size every four months and usually requires approximately nine years to achieve a mass of $2 \mathrm{~cm}$ in diameter.

\subsubsection{Adenocarcinoma}

Adenocarcinoma usually presents in two forms: 1) bronchial-derived adenocarcinoma, and 2) bronchoalveolar carcinoma, arising from terminal bronchioles or alveolar walls. Although there is overlap between these two forms, bronchoalveolar carcinoma has distinctive gross, microscopic and epidemiologic features. Adenocarcinoma is the most common type of 
bronchogenic carcinoma in women and non-smokers. Lesions are more peripherally located and tend to be smaller. They tend to grow more slowly than squamous cell carcinoma. Adenocarcinoma, including bronchoalveolar carcinoma, is less associated with a history of smoking than are squamous cell or small cell carcinoma.

\subsubsection{Small Cell Carcinoma}

Small cell carcinomas are highly malignant tumours. The epithelial cells are usually small and have little cytoplasm. Cells are round or oval, which is characteristic of oat cell carcinoma. Other small cells are spindle-shaped or polygonal-shaped and may be classified as spindle or polygonal small cell carcinoma. The cells grow in clusters that exhibit neither glandular nor squamous organization. Electron microscope studies show dense core neurosecretory granules in some of these tumours cells. These suggest derivation of these tumours from neuroendocrine cells of the bronchial epithelium lining. These tumours have a strong relationship to cigarette smoking. Only about $1 \%$ occurs in non-smokers. These tumours are usually located in hilar or central regions and are the most aggressive type of lung tumours. They metastasize widely and generally are incurable by surgical means.

\subsubsection{Small Cell Carcinoma}

0Small cell carcinomas are highly malignant tumours. The epithelial cells are usually small and have little cytoplasm. Cells are round or oval, which is characteristic of oat cell carcinoma. Other small cells are spindle-shaped or polygonal-shaped and may be classified as spindle or polygonal small cell carcinoma. The cells grow in clusters that exhibit neither glandular nor squamous organization. Electron microscope studies show dense core neurosecretory granules in some of these tumours cells. These suggest derivation of these tumours from neuroendocrine cells of the bronchial epithelium lining. These tumours have a strong relationship to cigarette smoking. Only 
about $1 \%$ occurs in non-smokers. These tumours are usually located in hilar or central regions and are the most aggressive type of lung tumours. They metastasize widely and generally are incurable by surgical means.

\subsubsection{Large Cell Carcinoma}

Large cell carcinoma has larger, more polygonal cells and vesicular nuclei. They probably represent undifferentiated squamous and adenocarcinoma. Some tumours contain mucin, some have a larger number of multinucleated cells (giant cell carcinoma), some have clear cells and are termed clear cell carcinoma, and others have a distinctly spindly histological appearance .(spindle cell carcinoma)

\subsection{Secondary Pathology}

Bronchogenic carcinomas cause related anatomical changes in the lung distal to the point of bronchial involvement. Partial obstruction may cause marked focal emphysema and total obstruction may lead to atelectasis. The impaired drainage of the airways is a common cause for severe suppurative or ulcerative bronchitis or bronchiectasis. Pulmonary abscesses sometimes draw attention to an occult carcinoma that has become infected. Compression or invasion of the superior vena cava can cause venous congestion, dusky head and arm edema, and ultimately circulatory compromise, known as the superior vena cava syndrome. Extension to the pericardium or pleural sac may cause pericarditis or pleuritis with development of significant effusions.

\subsection{Lung cancer Staging}

A uniform TNM system for staging cancer according to its anatomical extent at the time of diagnosis is important. The "T" descriptor indicates the extent of the primary tumour, "N" the 
extent of lymph node involvement, and " $\mathrm{M}$ " the presence of metastases. $(23,25)$ Table 1.1 below shows the staging system in its current form:

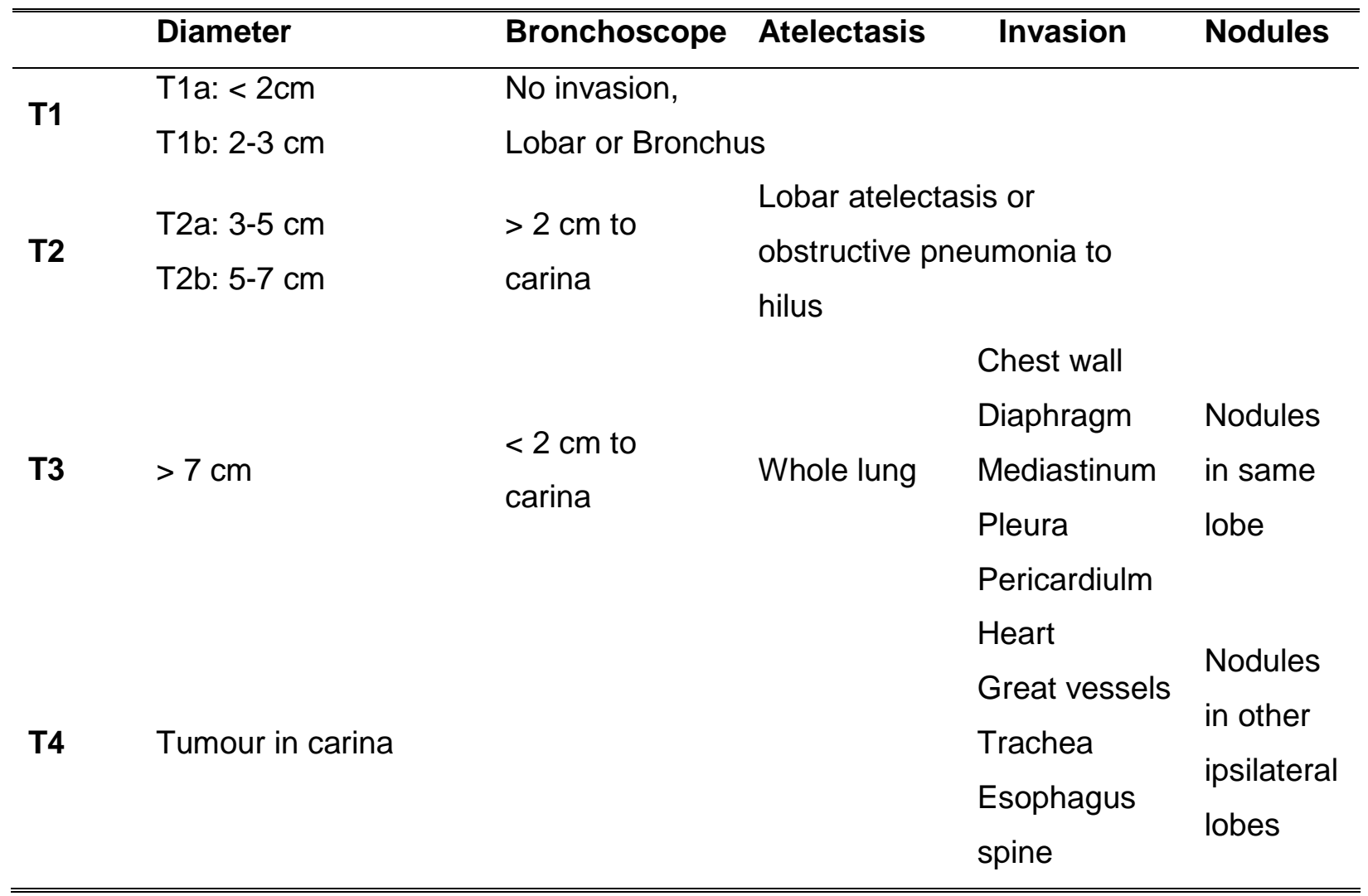

\begin{tabular}{ll}
\hline \hline N0 & No lymph nodes involved \\
N1 & Involvement of ipsilateral bronchopulmonary or hilar nodes \\
N2 & Involvement of ipsilateral mediastinal,ipsilateral suprclavicular or subcarinal nodes \\
N3 & Involvement of contralateral mediastinal, hilar, or supraclavicular lymph nodes \\
\hline \hline
\end{tabular}

M0 No distant metastasis

M1a: Separate tumour in a contralateral lobe or tumour

M1 Distant metastasis with plural nodules or malignant plural or pericardial effusion

M1b: Distant metastasis 
Table 1.1 TMN staging. The TNM staging system takes into account the degree of spread of the primary tumour, represented by $\mathrm{T}$; the extent of regional lymph node involvement, represented by $\mathrm{N}$; and the presence or absence of distant metastases, represented by $\mathrm{M}$.

The TNM staging is commonly grouped into subsets with similar prognosis and treatment options. These are grouped into four stages, as described in table 1.2

\begin{tabular}{|c|c|c|c|}
\hline Stage & Tumour & Lymph Node & Metastasis \\
\hline Stage IA & T1a,T1b & No & MO \\
\hline Stage IB & $\mathrm{T} 2 \mathrm{a}$ & No & MO \\
\hline \multirow[t]{3}{*}{ Stage IIA } & T1a,T1b & N1 & MO \\
\hline & $\mathrm{T} 2 \mathrm{a}$ & $\mathrm{N} 1$ & MO \\
\hline & $\mathrm{T} 2 \mathrm{~b}$ & No & MO \\
\hline \multirow[t]{2}{*}{ Stage IIB } & $\mathrm{T} 2 \mathrm{~b}$ & $\mathrm{~N} 1$ & MO \\
\hline & T3 & No & MO \\
\hline \multirow[t]{3}{*}{ Stage IIIA } & T1a,1b,T2a,T2b,T3 & N2 & MO \\
\hline & T3, T4 & $\mathrm{N} 1$ & MO \\
\hline & $\mathrm{T} 4$ & No & MO \\
\hline \multirow[t]{2}{*}{ Stage IIIB } & Any $T$ & N3 & MO \\
\hline & $\mathrm{T} 4$ & N2 & MO \\
\hline Stage IV & Any $\mathrm{T}$ & Any $\mathrm{N}$ & M1 \\
\hline
\end{tabular}

Table 1.2 Subtypes of lung cancer based on therapeutic and prognostic implication.

\subsection{Clinical Course}

Lung cancer is one of the most insidious neoplasms in the world of oncology. It may present with six to seven months of symptom duration. The major presenting complaints are a cough (75\%), weight loss (40\%), chest pain (40\%) and dyspnea (20\%). Increased sputum production is common and often contains diagnostic tumour cells when examined as cytologic specimens. 
Similarly cytologic examination from a fine needle aspiration (FNA) of a mass can often provide the diagnosis.

The outlook is generally poor for most patients with bronchogenic carcinoma. Despite all the efforts at early diagnosis by frequent radiological exams of the chest, cytological examination of the sputum, bronchial washing and brushing and many other improvements in thoracic surgery, radiotherapy, and chemotherapy, the overall five-year survival rate in united state is about $15 \%$ and in Europe and developing country is only $8 \%$ (25). In general, adenocarcinoma and squamous cell carcinoma tend to remain localized longer and have slightly better prognosis than undifferentiated tumours. Surgery is generally not curative for small cell carcinoma. If untreated, survival length is about 6-17 weeks, but this cancer is particularly sensitive to chemotherapy therefore providing temporary symptomatic relief in suitable patients. Unfortunately, most of these patients have distant metastasis at the time of diagnosis. However, earlier diagnosis of symptomatic patients with an earlier disease stage and use of multimodality therapy has shown promising outcomes in selective sub groups of patients.

Bronchogenic carcinoma has been associated with a number of paraneoplastic syndromes. The hormones or hormone-like factors elaborated include 1) antidiuretic hormones (ADH) inducing hypothermia, due to inappropriate $\mathrm{ADH}$ secretion, 2) adrenocorticotropic hormone (ACTH), producing Cushing syndrome, 3) parathormone, parathyroid hormone-related peptide, prostaglandin E, and some cytokines, all of which are implicated in hypercalcemia and sometimes seen in lung cancer, 4) calcitonin causing hypocalcemia, 5) gonadotropin causing gynecomastia, and 6) serotonin, associated with carcinoid syndrome. Any one of the histological types may occasionally produce any ectopic hormone, but ACTH and ADH are predominantly produced by small cell carcinoma, whereas hypercalcemia is usually related to squamous cell 
tumours. Carcinoid syndrome is rarely associated with small cell carcinoma but is more common with bronchial carcinoid.

Other systemic manifestations of lung tumours include Lambert-Eaton myasthenic syndrome, where muscle weakness is caused by autoimmune antibodies directed to t-neural calcium channels, peripheral neuropathy, usually purely sensory, dermatologic abnormalities, including acanthosis nigricans, hematologic abnormalities such as a leukemoid reaction, and finally a peculiar abnormality of connective tissue called hypertrophic pulmonary osteoarthropathy, associated with clubbing of the fingers.

Apical lung cancer in the superior pulmonary sulcus tends to invade the neural structures around the trachea, including the cervical sympathetic plexus, and produces a group of clinical findings that includes severe pain in the distribution of the ulnar nerve and Horner's syndrome (exophthalmos, ptosis, miosis, and anhidrosis) on the same side as the lesion. These tumours are also referred to as Pancoast tumours.

\section{Chest Imaging Technologies \\ 5.1 Chest Radiography (CXR)}

Chest radiography (CXR) is usually the first imaging step in evaluating patients with signs and symptoms of lung cancer. It presents a low-cost, minimal radiation dose modality that remains the mainstay of non-invasive examination and diagnosis of all chest pathologies in large health centers and rural hospitals alike. 

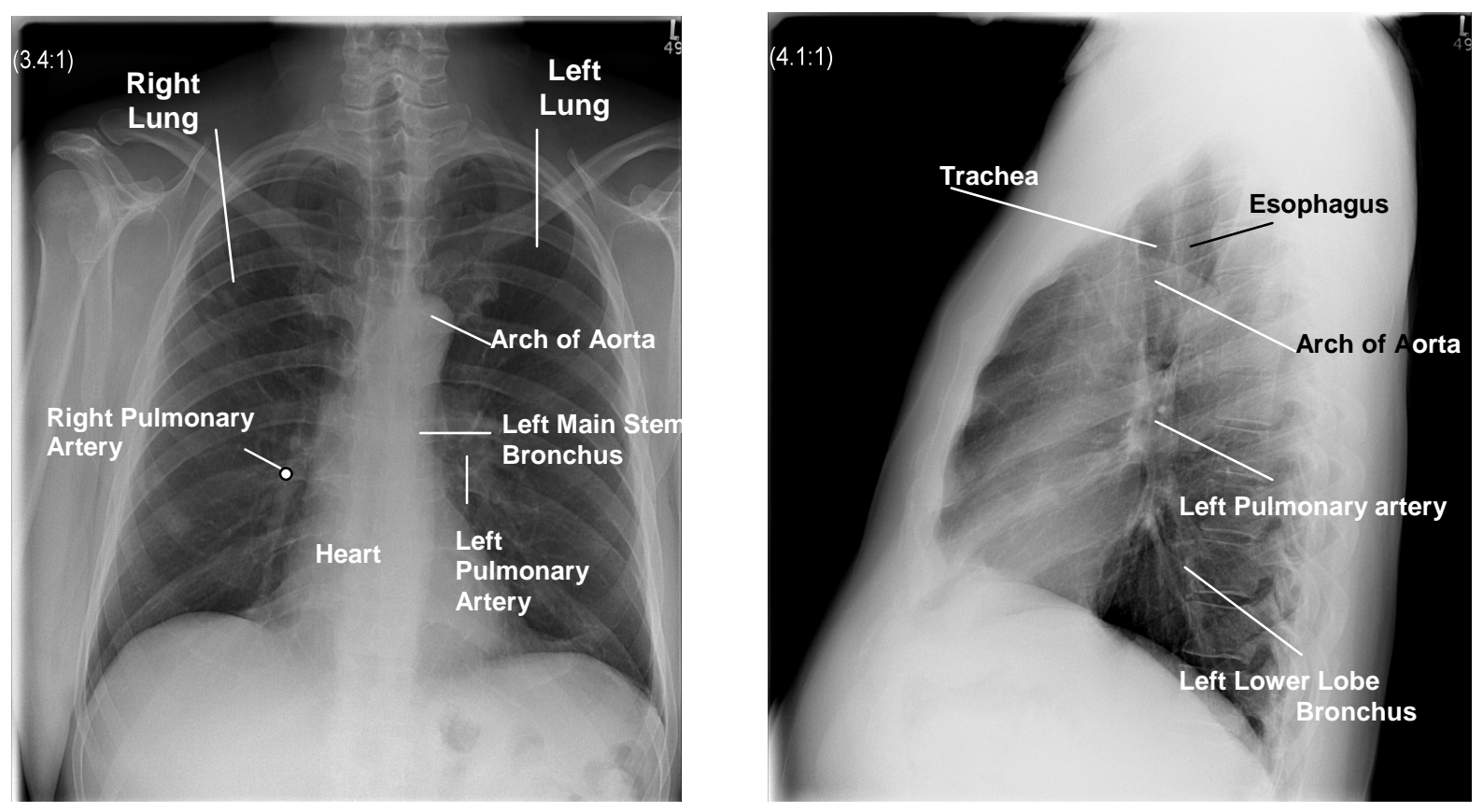

Figure 1.4 The anteroposterior (a) and lateral (b) views of male chest x-ray. Main anatomical regions are labeled.

Chest radiography has significant limitations due to the superimposition of anatomical structures. This is one of the main reasons why CXR lacks sensitivity in detection of small lung nodules, and has not shown itself to be suitable for screening purposes. (26) Technical advances in digital radiography (DR) have yielded remarkable improvements in diagnostic quality through the introduction of high-performance flat-panel detectors (FPDs). (27-29)

Today, conventional analogue chest imaging has been replaced by digital imaging at most medical centers. Digital image acquisition allows immediate electronic distribution of images through the use of a picture archiving and communication system (PACS). Digital imaging modalities include computed radiography (CR) and direct capture radiography (DR) imaging of lung disease. Still, the fundamental limitation to diagnostic performance remains which is the compression of three-dimensional anatomy into a two-dimensional projection image, (27) with the visual clutter associated with superimposed structures believed to be a major limiting factor. 
These limitations make planar radiography an unreliable technique for pulmonary nodule detection. Austin et al. (30) reviewed 27 cases of resectable lung cancer that were missed on previous chest radiographs. The majority of missed nodules were located in upper lobes and in females. Muhm et al (31) showed that $90 \%$ of peripheral nodules detected during a lung cancer screening program in retrospect were visible on earlier radiographs.

\subsection{Low Dose CT}

Computed tomography (CT), especially with helical and multi-detector technology, is superior to chest x-radiography for detecting pulmonary nodules. However, there is concern for the higher radiation exposure to the patient, compared with chest radiography, and this creates anxiety regarding the potential induction of malignancy (32).

The reported effective radiation dose in one routine computed tomography chest exam is several hundred times more than that of a single frontal chest radiograph ( $8 \mathrm{mSv}$ versus $0.02 \mathrm{mSv})(33)$. However, with modern CT units and more awareness of radiation dose issues, the radiation dose associated with thoracic CT has significantly decreased but still remains significantly higher than chest radiography (1.0 $\mathrm{mSv}$ for low dose CT). Low dose computed tomography has been shown to be a useful tool in the early detection of lung cancer (34). However, a low radiation dose causes increased image noise and image artifacts, which degrades image quality, especially in larger patients. Development of reconstruction software like special frequency filters, artifact correction and noise reduction algorithms have reduced such effects to some degree (35).

Although recent technological developments like volumetric scanning in a single gantry rotation offer faster scan times, reduced motion artifact and radiation dose, there are still some obstacles in using CT, which include high number of false positives and very high financial cost. 

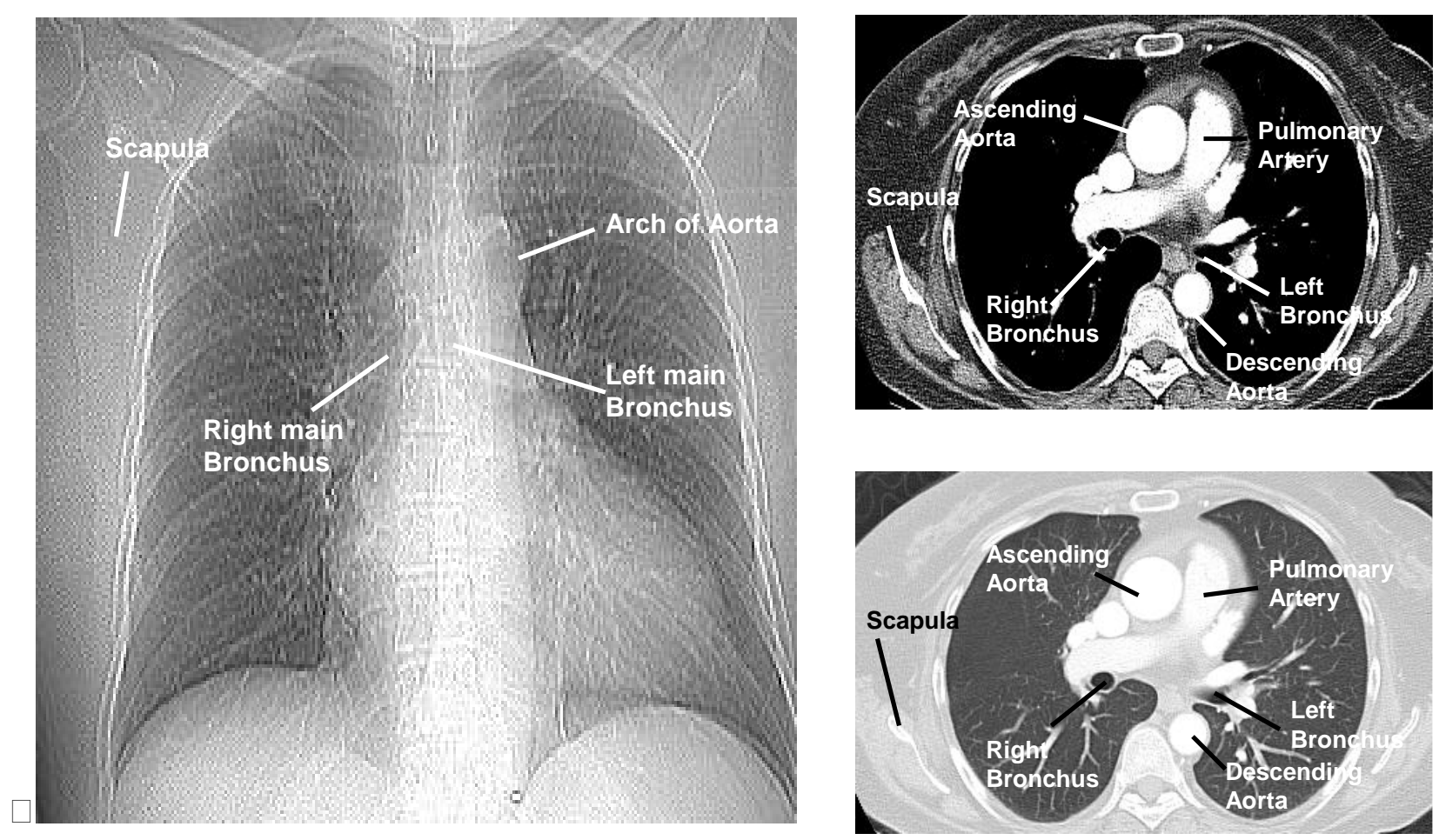

Figure 1.5 Comparison of the anatomical detail displayed on a frontal chest radiograph and an axial projection from a thoracic CT study taken at the level of the main pulmonary trunk.

\subsection{Positron Emission Tomography}

18F-fluorodeoxyglucose (FDG) positron emission tomography (PET) is a nuclear medicine imaging technique with evolving potential. 18F-fluorodeoxyglucose is a radiolabeled glucose analogue that is injected intravenously as the tracer. It is taken up by metabolically active cells, including cancer cells, and is phosphorylated in the intracellular space to FDG-6-Phosphate. This compound cannot get further catabolized and therefore remains trapped inside the cell. The trapped FDG decays by positron emission, the positron collides with an electron and produces energy in the form of a gamma ray, which can be detected by a tomography camera. The resultant images can be displayed in sagittal, axial or coronal planes. (36) FDG-PET measures the metabolic activity in a lung nodule/mass and can be very useful in determining the presence of cancer cells in indeterminate lung nodules and the presence of occult metastases. Therefore, 
FDG-PET is useful in detecting and staging lung cancer in earlier stages, however, it is associated with a high radiation dose especially when it is combined with CT as usually is the case in modern practice (37) Major reductions in radiation doses from PET/CT scans can be achieved by modifying the acquisition parameters for CT. Also, PET has the potential to assess tumour responsiveness to chemotherapy, which could serve as a prognostic factor and could influence further management of the disease. (38)

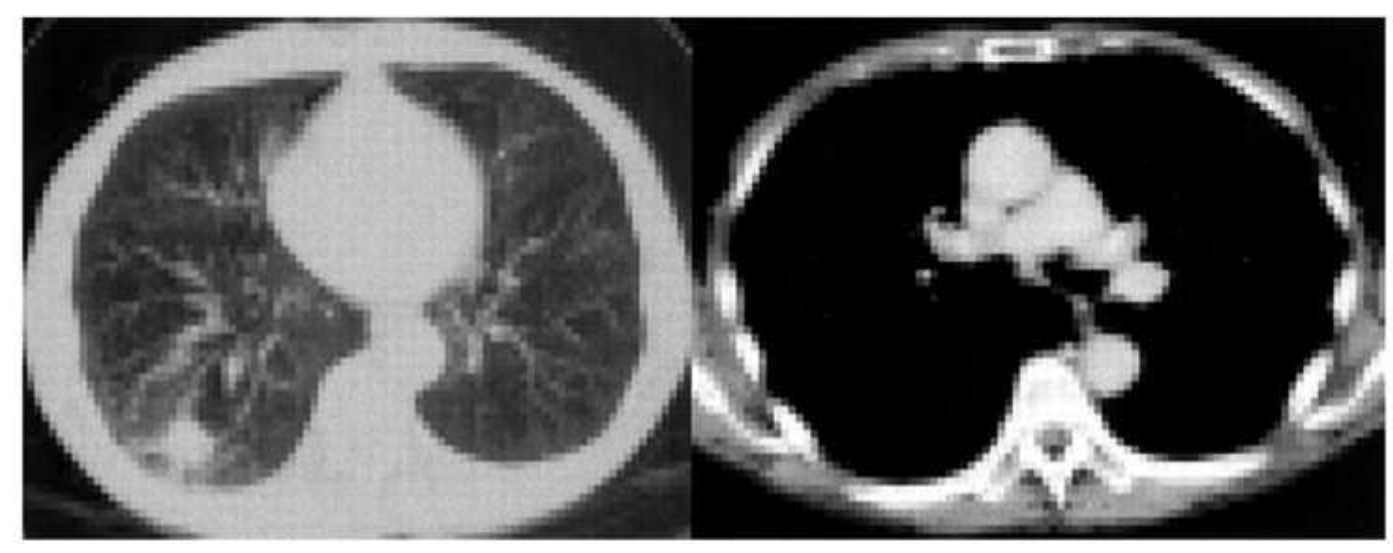

\section{FDG}

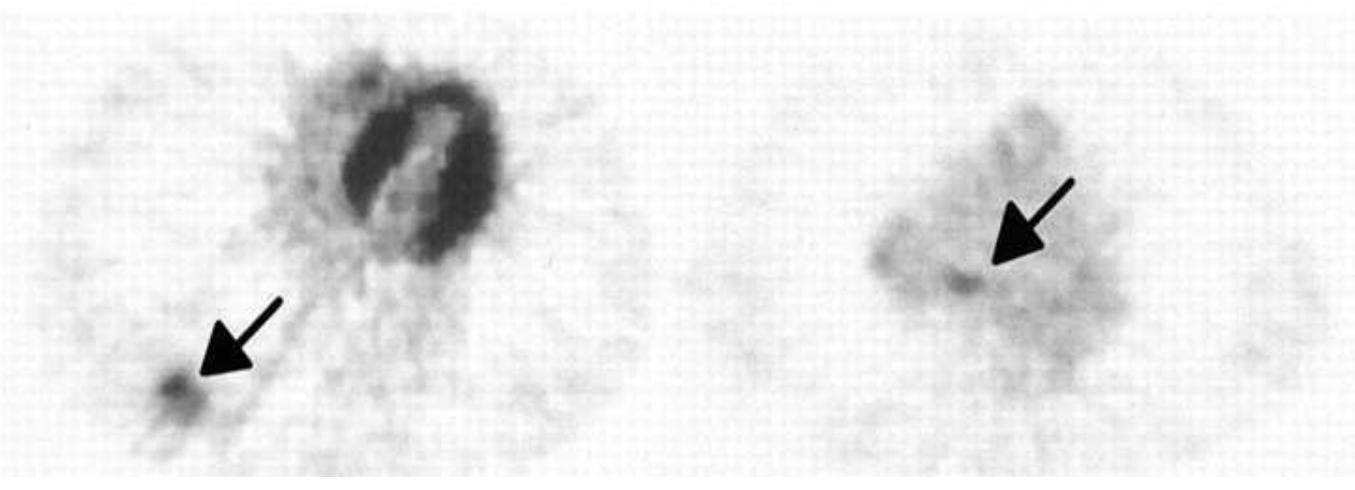

Figure 1.6 Projection images from a FDG PET scan (lower row) demonstrate intense uptake within a right lower lobe tumour. The top row shows the corresponding CT images (lung reconstruction (left) and mediastinal reconstruction, (right)). The tumour is seen at the same location. Modified from www.chestjournal.org/.../124/3/893/F1.expansion 


\subsection{Dual Energy Chest Imaging}

Dual Energy (DE) imaging acquires two projections of the patient at different x-ray energies and selectively decomposes the image into soft tissue and bone components. The former presents soft tissue structures in a context that is largely free of the main source of anatomical clutter (the ribs and clavicles), thus improving the conspicuity of subtle nodules. Furthermore, because the presence of calcification is an important indicator of benignancy, DE imaging could help characterize benign lesions with a higher level of specificity. Dual energy imaging may mitigate the limitations of anatomical clutter found in regular chest x-rays and also increase specificity by obtaining tissue-specific images. (39)

The general form of weighted log-subtraction decomposition of a DE image is as follow:

$$
\log \left(I_{D E}\right)=\log \left(I_{H}\right)-w \log \left(I_{L}\right)
$$

Where $\mathrm{I}^{\mathrm{H}}$ is the high energy image and $\mathrm{I}^{\mathrm{L}}$ is the low energy image. $\mathrm{W}$, the weighting factor is the ratio of the attenuation coefficient of the cancelled material at low and high energy. This is described in details in the next chapter.

Commercially available DE imaging systems include storage phosphor and (Flat panel detector) FPD designs. Single-shot DE imaging (e.g., FCR XU-D1; Fujifilm, Tokyo, Japan) involves two CR storage phosphors as front and back detectors separated by an intermediate filter, with the image produced by the front plate representing a low-energy image and that by the back plate a high-energy image. As detailed below, soft tissue and bone images are decomposed from these low- and high-energy images using various subtraction algorithms. Double-shot DE images are generated using two x-ray exposures at two kVp settings (e.g., QX/I Revolution, GE Healthcare, Chalfont St. Giles, UK). The advantage of this system is the improved energy separation (and, therefore, contrast-to-noise ratio) compared to single shot DE designs but the potential 
disadvantage of two acquisitions lies in the patient related motion artifacts (breathing, moving and cardiac) which happen due to the time interval between the two exposures, even for FPDs with rapid readout. Although a compliant patient may be able to hold their breath and not move between the two exposures, there is usually a change in heart rate, even with a short breath hold, therefore accurate and robust cardiac gating would be a useful feature for two shot DE acquisitions.

\subsection{Radiation Hazard}

Potential long term health effects, such as cancer induction and hereditary genetic damage, caused by exposure to ionizing radiation are not well understood but have been identified. It is assumed that the relationship between radiation exposure and adverse effect is linear, meaning that a doubling of radiation dose results in twice the risk of damage. In addition, many health scientists assume that there is no radiation dose threshold for these ill effects, meaning that there is no level of energy at which the health risk is assumed to be zero. (40) The advent of computed tomography has revolutionized diagnostic radiology but CT involves larger radiation doses than conventional chest radiography. Multiple factors are involved in determining the radiation dose to a particular organ during CT imaging, including the tube potential in kilovolts $(\mathrm{kVp})$, the tube current in milliamperes (mA), the scan time in second (s) and the size of the patient. The scan parameters should be tailored to the study protocol in order to minimize patient radiation exposure.

The principle reason that ionizing radiation is a biological hazard relates to the unique energy of the X-ray photons which is sufficient to overcome the binding energy of the electrons orbiting the atomic nucleus and thereby displace them from their orbits. The residual atomic nucleus becomes a positively charged ion and this entity can interact with DNA. The most commonly 
created ions are hydroxyl radicals from interaction of X-ray photons with water molecules. These radicals can cause breakage of the DNA double helix strands or can ionize DNA proteins directly. The break in the double stranded DNA is not easily repaired and occasionally the misrepair can induce point mutations and chromosomal translocations which potentiate cancer cells.

Most of the available data for estimating the risk of radiation induced cancer comes from extensive studies performed on the Japanese survivors of atomic bombs explosions. The risk from exposure to ionizing radiation was quantitatively consistent in all of these studies. Children are at greater risk than adults from a given dose of radiation since they are more radiosensitive and they also have more remaining years of life, in which to develop induced cancer, compared to adults. (42) Overall it is possible to estimate the cancer risks associated with radiation exposure by estimating the organ doses involved and applying organ specific cancer incidence or mortality data.

The estimated number of CT scans that lead to cancer, varies depend on the type of the scan and the patient sex and age. For example according to one study published by Smith et al (43) the estimated risk of cancer induction in women undergoing CT coronary angiography at the age of 40 years, is 1 in 270 . The analysis resulted in the rate of 1 in 600 for men. For head CT the same analysis resulted in an estimated cancer rate of 1 in 8100 for women and 1 in 11080 for men. For 20 year old patients the overall risk was almost doubled and for 60 year old patients, it was $50 \%$ lower.

Bearing this in mind, an important pre-requisite for this research was to ensure that the radiation dose associated with the dual energy exposure was equivalent to that from a conventional frontal chest radiography $(0.02 \mathrm{mSv})$. 


\section{Chapter 2 \\ Dual Energy Prototype and Patient Cohort \\ 1 DE Imaging System}

The experimental prototype DE imaging system was based on a Kodak RVG 5100 digital radiography chest stand (Carestream Health Inc., Rochester, NY). An acquisition workstation controls generator technique setting, filter selection, detector acquisition parameters, and data transfer. The system was modified for the purposes of DE imaging to include: 1) a flat-panel detector (Trixell Pixium-4600, Moirans, France) with a $\sim 250 \mathrm{mg} / \mathrm{cm}^{2}$ CsI:Tl scintillator and 143 $\mu \mathrm{m}$ pixel pitch, 2) a fingertip pulse oximeter to trigger $\mathrm{x}$-ray exposure during diastole for reduction of cardiac motion artifacts, (44) and 3) a multi-position filter wheel within the collimator to change the added filtration between the low-kVp $(2.5 \mathrm{~mm} \mathrm{Al})$ and high-kVp (2 mm $\mathrm{Al}+0.6 \mathrm{~mm} \mathrm{Ag}$ ) exposures, as guided by previous studies of DE image quality performed in our laboratory. (44-46)

The X-ray tube produces a polychromatic beam with spectrum of beam energies distributed around the pre set target tube potential. Therefore, a high-energy filter was selected to "harden" the high energy beam, to reduce spectral overlap between the low- and high-energy projections. This was the subject of considerable investigation in our laboratory $(45,46,47)$. Differential filtration between low- and high-kVp projections was selected and an extensive analysis of contrast, Noise Equivalent Quanta (NEQ) and nodule detectability for low- and high -kVp added filtration was performed. A greater than $50 \%$ improvement in contrast and a boost in NEQ were achieved using an additional $0.6 \mathrm{~mm} \mathrm{Ag}$ in the high-kVp projection. (45) The added filtration for the DR image was typical of that used in conventional digital chest radiography. Three different 
filters for the low-energy, high-energy, and DR exposures were implemented using a computercontrolled, multi-position filter wheel within the collimator.

Optimal acquisition techniques including $\mathrm{kVp}$ pair and dose allocation (Ać) were identified in previous work by experimental measurements in phantoms (46) and theoretical analysis. (48) A chest phantom with simulated lung nodules was used. For thick $(30 \mathrm{~cm})$, average $(24 \mathrm{~cm})$ and thin $(18 \mathrm{~cm})$ patients, various thickness of acrylic was used. Multiple acquisition were taken using a range of low-kVp (60-90 kVp), high-kVp (120-150 kVp) and dose allocation $(\mathrm{A} \varepsilon \dot{\varepsilon}=\sim 0$ 1). Patient dose, characterized as total imparted energy $\left(\dot{\varepsilon}^{\text {Total }}=\dot{\varepsilon}^{\mathrm{L}}+\dot{\varepsilon}^{\mathrm{H}}\right)$ was kept equal to that of a single DR radiograph (within $\pm 5 \%$ ) for the same chest thickness. DE images were acquired at various low-kVp, high-kVp, and allocations. For each patient thickness, and $\mathrm{kVp}$ pair, combinations of $\dot{\varepsilon}^{\mathrm{L}}(\mathrm{mAs})$ and $\dot{\varepsilon}^{\mathrm{H}}$ (mAs) that yielded a given total dose ( $\left.\dot{\varepsilon}^{\text {Total }}\right)$ equal to one DR (within $\pm 5 \%$ ) were identified. The total of ten combinations were identified for each patient thickness and $\mathrm{kVp}$ pair, since for each mAs combination, a specific allocation resulted in total dose equal to one DR. To identify the optimal $\mathrm{kVp}$ pair and allocation for each patient thickness, Signal difference to noise ratio (SDNR) was measured in DE soft tissue images of the phantom for a total of $16 \mathrm{kVp}$ pairs and three phantom thicknesses. (46) For each combination, the peak SDNR was found at an allocation of $A \dot{\varepsilon}=0.33$, suggesting optimal image quality when one third of the total dose is imparted by the low- $\mathrm{kVp}$ beam. Also a significant improvement in SNRD was observed with increasing the spectral separation, by reducing the low-kVp. The SDNR was found to be highest at $60 / 120 \mathrm{kVp}$. DR techniques for thin, average and thick patient sizes were obtained from the clinical technique chart in our institution. (46) 
The technique charts for DE and DR (Table 2.1,2.2) were interpolated across patient chest thicknesses ranging 18-28 $\mathrm{cm}$ in increments of $2 \mathrm{~cm}$, with the total dose dependent on patient thickness (e.g., $0.11 \mathrm{mGy}$ for $24 \mathrm{~cm}$ chest thickness).

\begin{tabular}{|c|c|c|c|c|c|c|c|}
\hline \multicolumn{2}{|c|}{ Optimal technique } & \multicolumn{3}{|c|}{$60 \mathrm{kVp}$} & \multicolumn{3}{|c|}{$120 \mathrm{kVp}$} \\
\hline $\begin{array}{c}\text { Chest } \\
\text { thickness } \\
\text { (cm) }\end{array}$ & $\begin{array}{c}\text { Range } \\
\text { (cm) }\end{array}$ & $\mathrm{mA}$ & ms & mAs & $\mathrm{mA}$ & ms & mAs \\
\hline 14 & $<15$ & 400 & 6.25 & 2.5 & 400 & 25 & 10.0 \\
\hline 16 & $15-16.9$ & 400 & 6.25 & 2.5 & 400 & 32 & 12.8 \\
\hline 18 & $17-18.9$ & 400 & 8 & 3.2 & 400 & 40 & 16.0 \\
\hline 20 & $19-20.9$ & 400 & 10 & 4.0 & 500 & 40 & 20.0 \\
\hline 22 & $21-22.9$ & 500 & 10 & 5.0 & 630 & 40 & 25.2 \\
\hline 24 & $23-24.9$ & 400 & 12.5 & 5.0 & 630 & 40 & 25.2 \\
\hline 26 & $25-26.9$ & 400 & 16 & 6.4 & 630 & 50 & 31.5 \\
\hline 28 & $27-28.9$ & 630 & 12.5 & 7.9 & 630 & 63 & 39.7 \\
\hline 30 & $29-30.9$ & 630 & 16 & 10.1 & 630 & 63 & 39.7 \\
\hline
\end{tabular}

Table 2.1 Optimal technique chart for Dual energy projections based on patient thickness.

\begin{tabular}{cccccc}
\hline \hline $\begin{array}{c}\text { Chest } \\
\text { Thickness }\end{array}$ & $\begin{array}{c}\text { Range } \\
(\mathbf{c m})\end{array}$ & $\mathbf{k V p}$ & $\mathbf{m A}$ & $\mathbf{m s}$ & $\mathbf{m A s}$ \\
$\mathbf{( c m )}$ & & & & & \\
\hline 14 & $<15$ & 120 & 32 & 50 & 1.6 \\
16 & $15-16.9$ & 120 & 32 & 50 & 1.6 \\
18 & $17-18.9$ & 120 & 40 & 50 & 2 \\
20 & $19-20.9$ & 120 & 40 & 50 & 2 \\
22 & $21-22.9$ & 120 & 40 & 63 & 2.5 \\
24 & $23-24.9$ & 120 & 40 & 80 & 3.2 \\
26 & $25-26.9$ & 120 & 50 & 80 & 4 \\
28 & $27-28.9$ & 120 & 63 & 80 & 5.0 \\
30 & $29-30.9$ & 120 & 80 & 80 & 6.4 \\
\hline \hline
\end{tabular}

Table 2.2 Optimal technique chart for digital radiograph projection based on patient thickness. 
DE images were processed and decomposed as illustrated in Fig. 2.1. Offset and gain corrections were based upon 25 averaged dark-fields and 25 averaged flood-fields (acquired at 50\% sensor saturation), respectively. Prior to DE decomposition, the high-energy image was automatically registered to the low-energy image by means of deformable registration based on mutual information maximization and a morphological pyramid. (49)

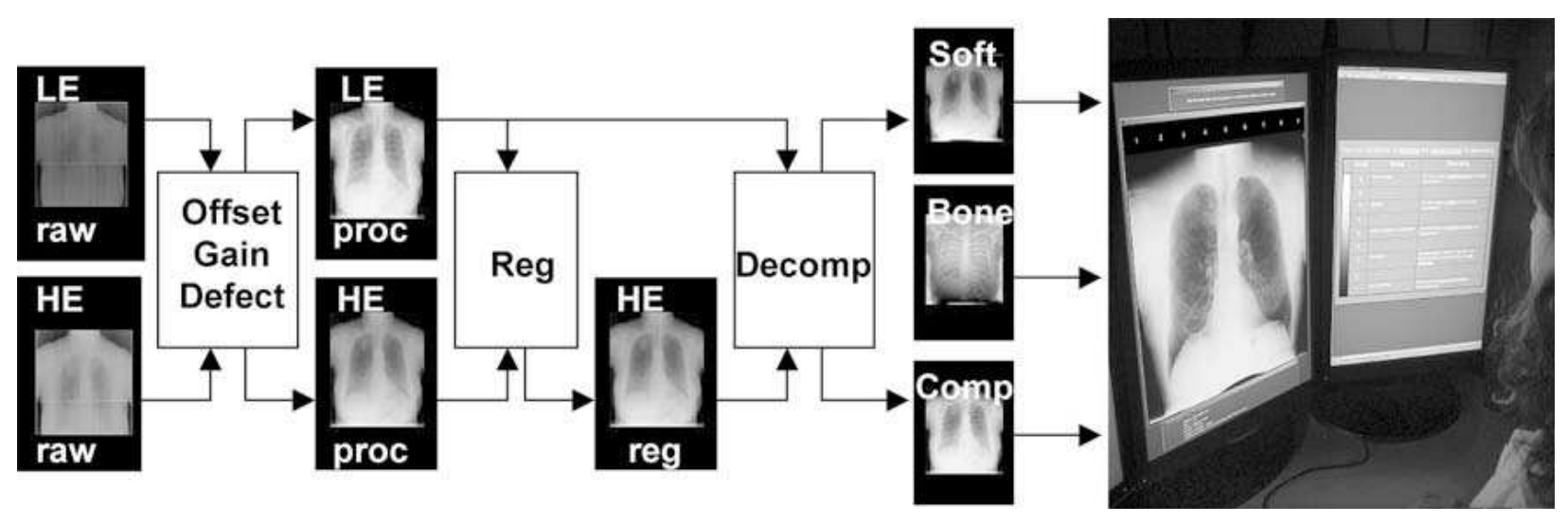

Figure 2.1 Illustration of dual-energy (DE) image processing, registration (Reg), decomposition (Decomp) and display. Processing steps include offset, gain, and defect correction; deformable registration; weighted log-subtraction decomposition; and the transformation of pixel values to log-exposure space for display. The photograph on the right shows the experimental setup for observer studies, with images displayed on the left and the rating scale on the right. Bone: bone decomposition; Comp: composite equivalent radiograph; HE: high-energy projection; LE: lowenergy projection; Soft: soft tissue decomposition.

The low-energy image and the (registered) high-energy image were decomposed into soft tissue and bone images by log-weighted subtraction of the basic form:

$\log \left(\mathrm{I}_{\text {soft }}\right)=\log \left(\mathrm{I}_{\mathrm{H}}\right)-\mathrm{W}_{\mathrm{s}} \log \left(\mathrm{I}_{\mathrm{L}}\right)$

$\log \left(\mathrm{I}_{\text {bone }}\right)=-\log \left(\mathrm{I}_{\mathrm{H}}\right)+\mathrm{W}_{\mathrm{b}} \log \left(\mathrm{I}_{\mathrm{L}}\right)$

$\log \left(\mathrm{I}_{\text {Com }}\right)=\log \left(\mathrm{I}_{\mathrm{H}}\right)+\mathrm{w}_{\mathrm{c}} \log \left(\mathrm{I}_{\mathrm{L}}\right)$ 
Where $\mathrm{I}_{\mathrm{H}}$ is the high energy image and $\mathrm{I}_{\mathrm{L}}$ is the low energy image. $W$ is the tissue cancellation parameter and ideally is the ratio of the attenuation coefficient $(\mu)$ of the cancelled material at low and high energy. For example:

$$
\begin{aligned}
& \mathrm{w}_{\mathrm{S}}=\mu \text { bone }_{\mathrm{H}} / \mu \text { bone }_{\mathrm{L}} \\
& \mathrm{w}_{\mathrm{b}}=\mu \operatorname{soft}_{\mathrm{H}} / \mu \text { soft }_{\mathrm{L}}
\end{aligned}
$$

This gives three components to each DE image: the soft-tissue image (DE soft), the bone image (DE bone), and the composite "equivalent radiograph" image (DE comp). The basic log subtraction technique of Eq. (1) was modified to include noise reduction techniques optimal to each image. (50) Specifically, the soft tissue image was decomposed using an anti-correlated noise reduction $(\mathrm{ACNR})$ algorithm, $(48,50,51)$ and the bone image was decomposed using a simple smoothing of the high-energy image (SSH) algorithm. $(48,52)$ The ACNR algorithm is based on the fact that quantum noise in the soft tissue image and bone only image is anticorrelated. In decomposing a soft tissue image ACNR applies a high pass filter to the complementary image (the bone only image) which removes the gross anatomical structures from the complementary image and leave it only with some residual edge artifact and quantum noise which is anti-correlated to the quantum noise in the original DE image. The original DE image and the filtered complementary image are then added and weighted by a parameter $w_{\mathrm{n} . .}$ Therefore the soft tissue image decomposed by ACNR is:

$$
\mathrm{I}_{\mathrm{ACNR}}=\mathrm{I}_{\mathrm{Soft}}+\mathrm{w}_{\mathrm{n}} \mathrm{I}_{\text {bone }} * \mathrm{~h}_{\mathrm{HPF}}
$$

Where $\mathrm{I}_{\text {bone }}=-\mathrm{I}_{\mathrm{H}}+\mathrm{W}_{\mathrm{b}} \mathrm{I}_{\mathrm{L}}$, is the complementary image and $\mathrm{w} \mathrm{b}$ is the tissue cancellation parameter. $\mathrm{h}_{\text {HPF }}$ is the high pass filter used for complementary image. $\mathrm{w}_{\mathrm{n}}$ can be determined qualitatively or quantitatively through the minimization of quantum noise. 
$\mathrm{SSH}$ is based on the proven fact that the greater contributor of the quantum noise in the resulting DE images is the high energy projection. This effect can be alleviated by applying a low pass filter to the high energy projection before log weighted subtraction. The unfiltered low energy projection preserves the high special frequency information. Therefore the resulting image is expressed as:

$$
\mathrm{I}_{\mathrm{SSH}}=-\mathrm{I}_{\mathrm{H}} * \mathrm{~h}_{\mathrm{LPF}}+\mathrm{w} \mathrm{I}_{\mathrm{L}}
$$

Where $h_{L P F}$ is the low pass filter, $I_{H}$ is the high energy image, $I_{L}$ is the low energy image and $\mathrm{w}$ is the cancellation parameter.

All decomposition parameters (ws, wb, wc, etc.) were manually selected for each case by a reader (who was not one of the observers in the observer tests). The reader was trained by a radiologist prior to modifying the images to learn the overall radiologists' preferences for the resulting soft and bone images. The main image quality criterion considered by the expert reader involved the overall completeness of tissue cancellation (e.g., extinction of bone in the soft tissue image and vice versa). The resulting soft tissue images were judged to maximize conspicuity of pulmonary structures (and minimize visibility of bone), and conversely for the bone image. The composite image was selected in a manner to give image quality, tissue contrast, etc. as close as possible to that exhibited in the DR image. The adjustments to each image were qualitative in nature, this was necessary in order to allow for variables including patient body shape and positioning. Example images are shown in Fig. 2.2

The DR image was acquired a few seconds after the DE image pair on the same imaging prototype. The technique for the DR image used a tube potential of $120 \mathrm{kVp}(1 \mathrm{~mm} \mathrm{Al}+0.2 \mathrm{~mm}$ 
$\mathrm{Cu}$ filtration), and the tube current (mAs) was set according to the PA chest thickness and determined from a published technique chart. (46)
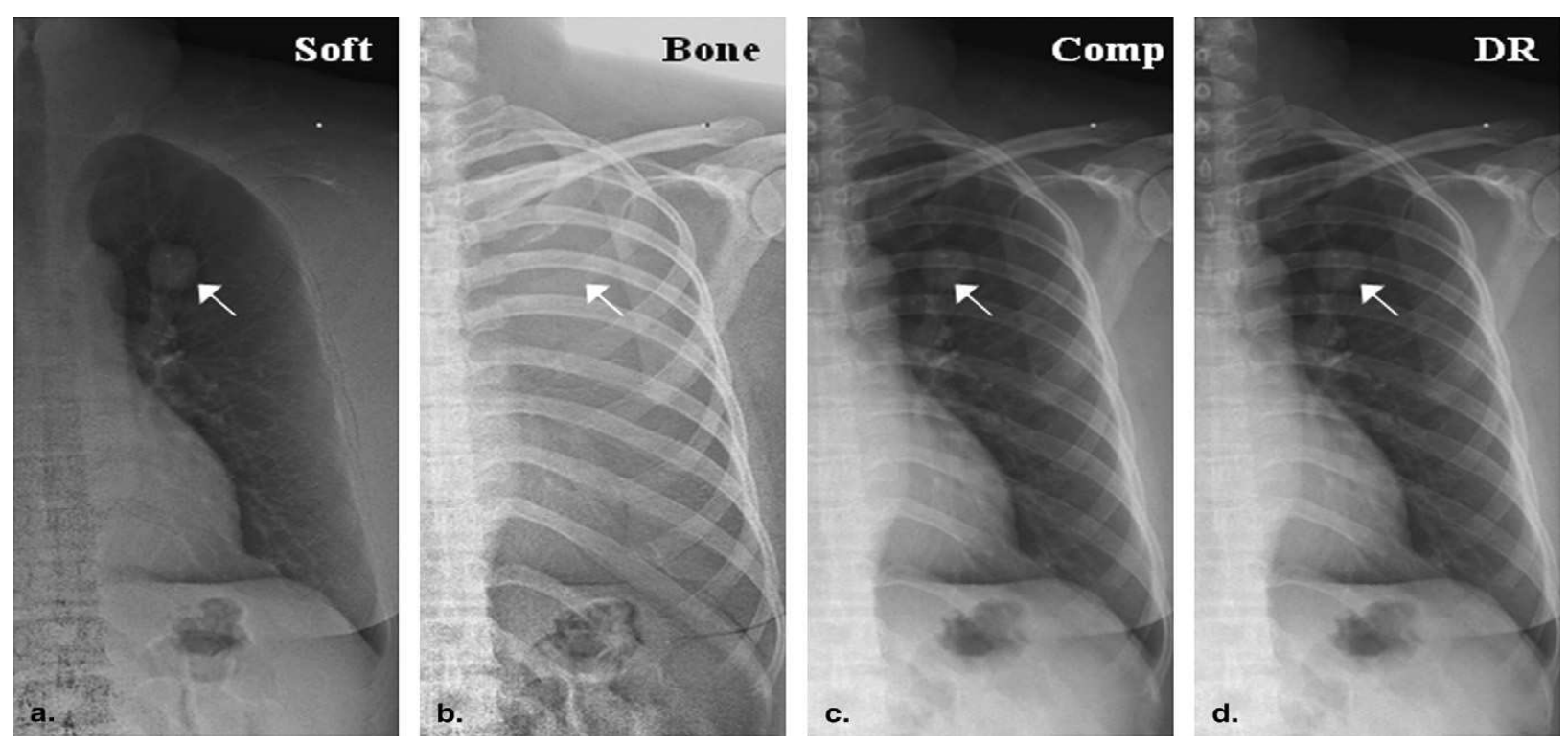

Figure 2.2 Example dual energy (DE) and digital radiography (DR) images. DE components include: (A) the soft-tissue image, $(B)$ the bone image, and $(C)$ the composite image. The corresponding DR image is shown in (D). The location of a $2.4 \mathrm{~cm}$ nodule in the left upper lobe is marked by an arrow.

\section{Patient cohort}

Patients were accrued under informed consent in a prospective, non-randomized trial with approval from the institutional research ethics board and with Health Canada Investigational Testing Authorization. The total study cohort of 220 patients was drawn from the patient population referred for a percutaneous computed tomography-guided biopsy of suspicious lung nodules between September 2006 and April 2008. Based on the study design, all patients had to have a previously detected indeterminate lung nodule, a standard dose thoracic CT and histology. Therefore, all of the patients had at least one suspicious lung nodule detected either on an initial computed tomography study or on chest radiography. 
Patients were prospectively accrued into five arms differing in DE imaging technique to evaluate different DE imaging parameters. These included differential x-ray spectra, cardiac gating, dose allocation, and total dose. (53) The main study cohort of 129 patients (80 M, $49 \mathrm{~F}, 62 \%$ male) with a mean age of 64.8 year was allocated to the optimal imaging technique. This gender distribution is reflective of the patient population referred to our Institution for lung biopsy during the period of the study (Sep 2006-Apr 2008, $56 \%$ males). The remaining patients were equally allocated to the other four groups. Figure 2.3 below shows the different group allocations.

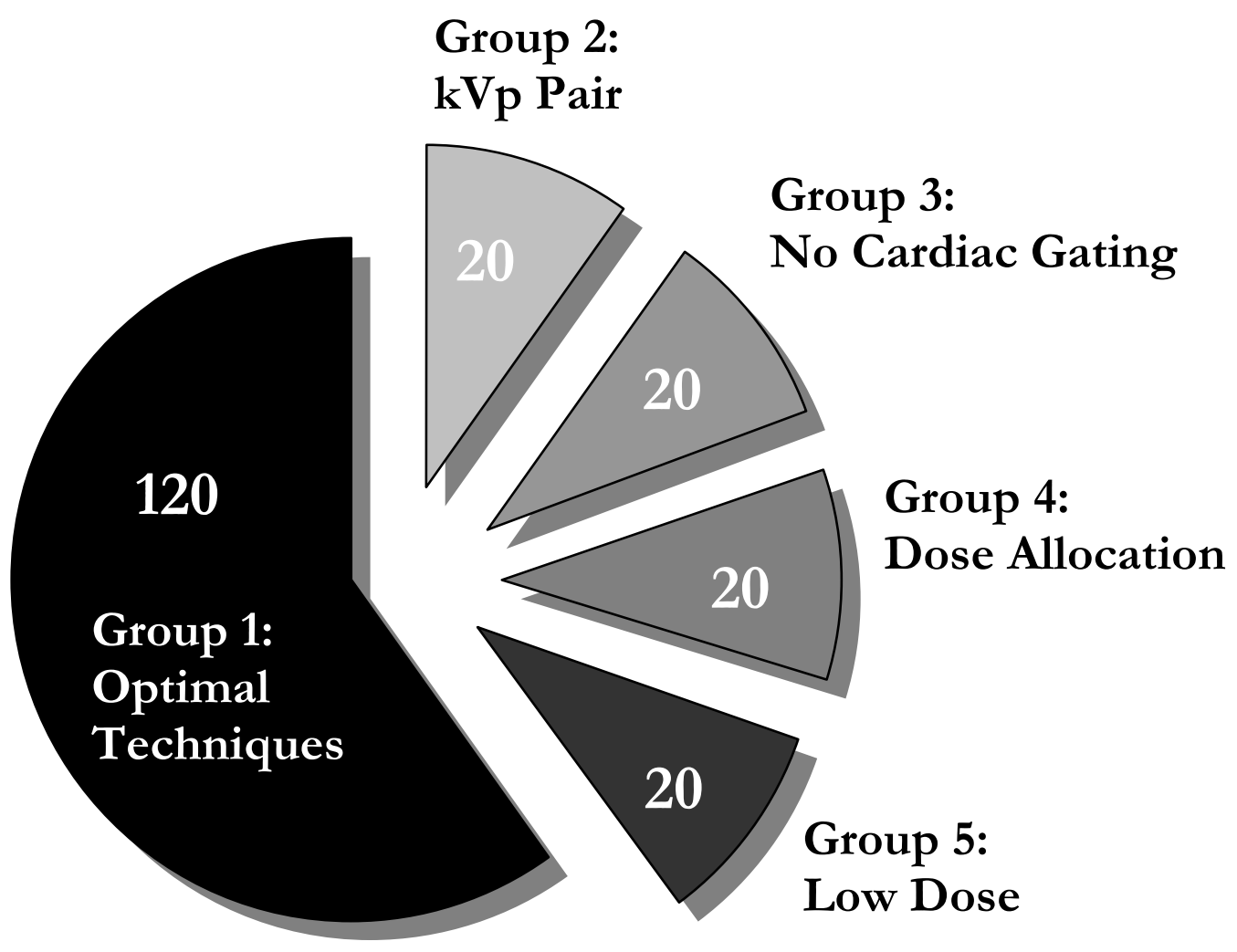

Figure 2.3 Patient accrual into five different research arms to evaluate variable DE parameters. 
The following data was gathered for each patient in the trial: standard-of-care image data (standard dose CT, ultra low-dose CT, acquired just prior to lung biopsy, and a post-biopsy CR image), experimental protocol image data (a frontal chest DR image and a DE image, each described below), and biopsy data. Percutaneous CT guided transthoracic biopsies were performed immediately following the DE/DR imaging exam to provide a definitive diagnosis of the lung lesion. Biopsies were performed either by fine-needle aspiration and cytologic examination or by core biopsy and histologic examination. (54)

Two different observer studies were performed. The first study incorporated a diagnostic satisfaction rating test using 55 cases (accrued at the time of the observer study) and the second study utilized Receiver Operating Characteristic (ROC) analysis for pulmonary nodule detection, using 129 cases. $(53,55)$ All images used in the two studies reported herein were taken from Group 1 (the “optimal” DE imaging technique described above).

\section{Meta Data}

Different parameters were measured for each patient. These parameters were grouped in five different categories:
A. Patient Characteristics
B. Disease Characteristics
C. Exam Characteristics
D. DE Image Techniques

E. Multi-Scale, Multi-Resolution Registration (MSMR) Characteristics 


\subsection{Patient Characteristics}

\subsubsection{Gender}

Patient gender was acquired from electronic patient information accessible to hospital employees. A total of 91 females and 129 males were accrued for the study.

\subsubsection{Age}

Patient age at the time of admission was acquired from the patient information system. The mean age was 64.9 years, ranging from 25 to 90 years; the frequency of cases in each age range is demonstrated in Figure 2.4. Most patients were in the 55-75 years age range and this accurately reflects the presenting age of patients with lung cancer.

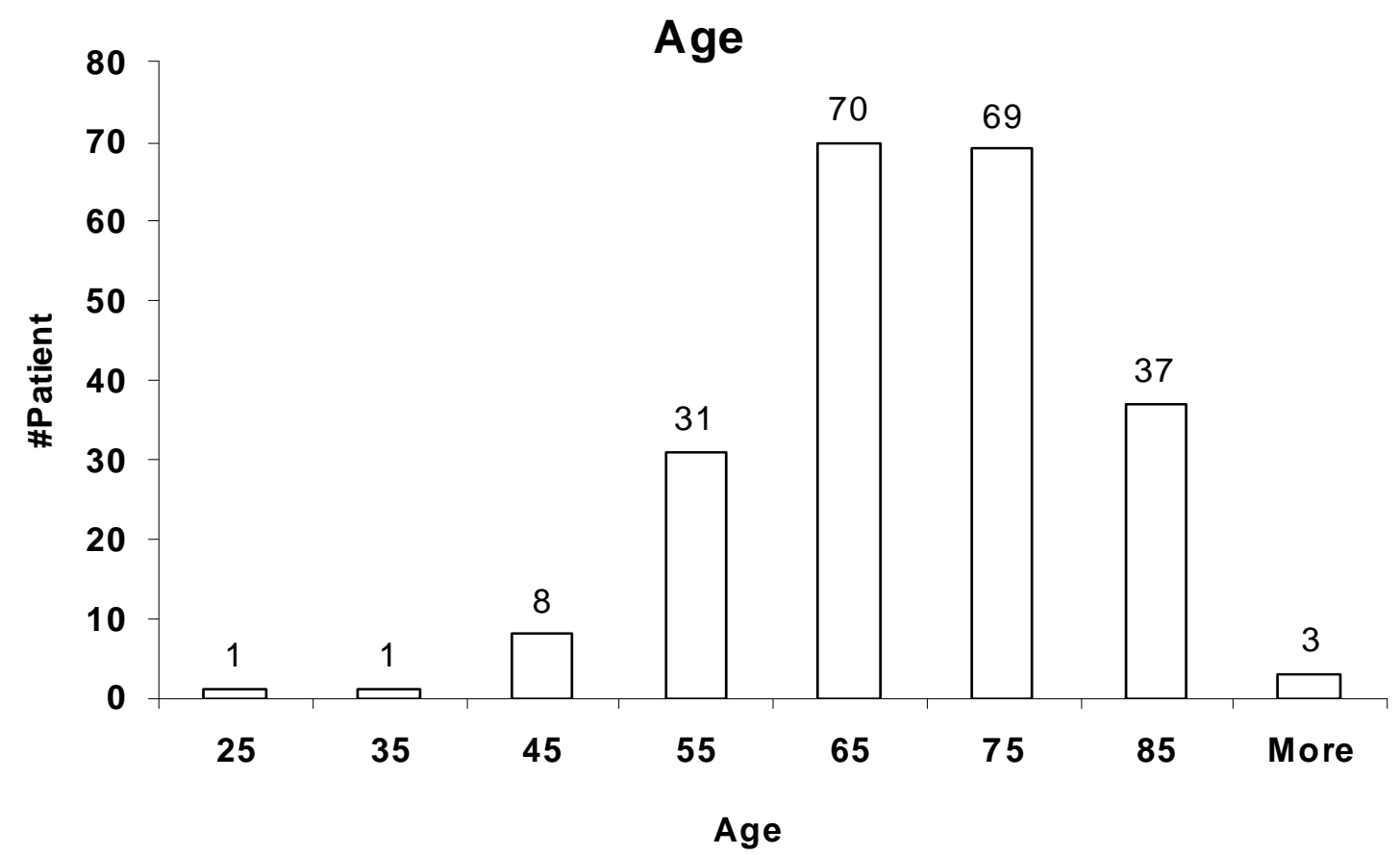

Figure 2.4 A histogram describing the number of cases for each age group. The majority of patient with suspicious lung nodules were between 55 and 65 years of age (70 Cases) followed by those between 65 and 75 years of age ( 69 cases). 


\subsubsection{Region}

Primary lung cancer most commonly presents in the upper lobes (56). To determine the most common location for the study population, the location of the lesion undergoing biopsy was recorded using the diagnostic CT axial projections that were taken prior to the lung biopsy.

Regions were divided into 7 different areas based on the anatomy of the lung: right upper lobe, right middle lobe, right lower lobe, left upper lobe, lingula, left lower lobe and mediastinum. Both lungs have an oblique fissure that separates the upper lobe, from the lower lobe. In addition, the right lung has a horizontal fissure that creates a middle lobe from the upper lobe. The middle lobe has an anatomical equivalent in the left lung, the lingual which has a dedicated bronchial anatomy but does not have a separate fissure. The mediastinum lies between the right and left pleura and is surrounded by loose connective tissue. It contains the heart, the great vessels of the heart, esophagus, trachea, phrenic nerve, cardiac nerve, thoracic duct, thymus, and lymph nodes of the central chest.

The number of cases in each category was plotted against the region. As demonstrated in Figure 2.5 below, the frequency of cases was highest in the right upper lobe and lowest in the left middle lobe. 


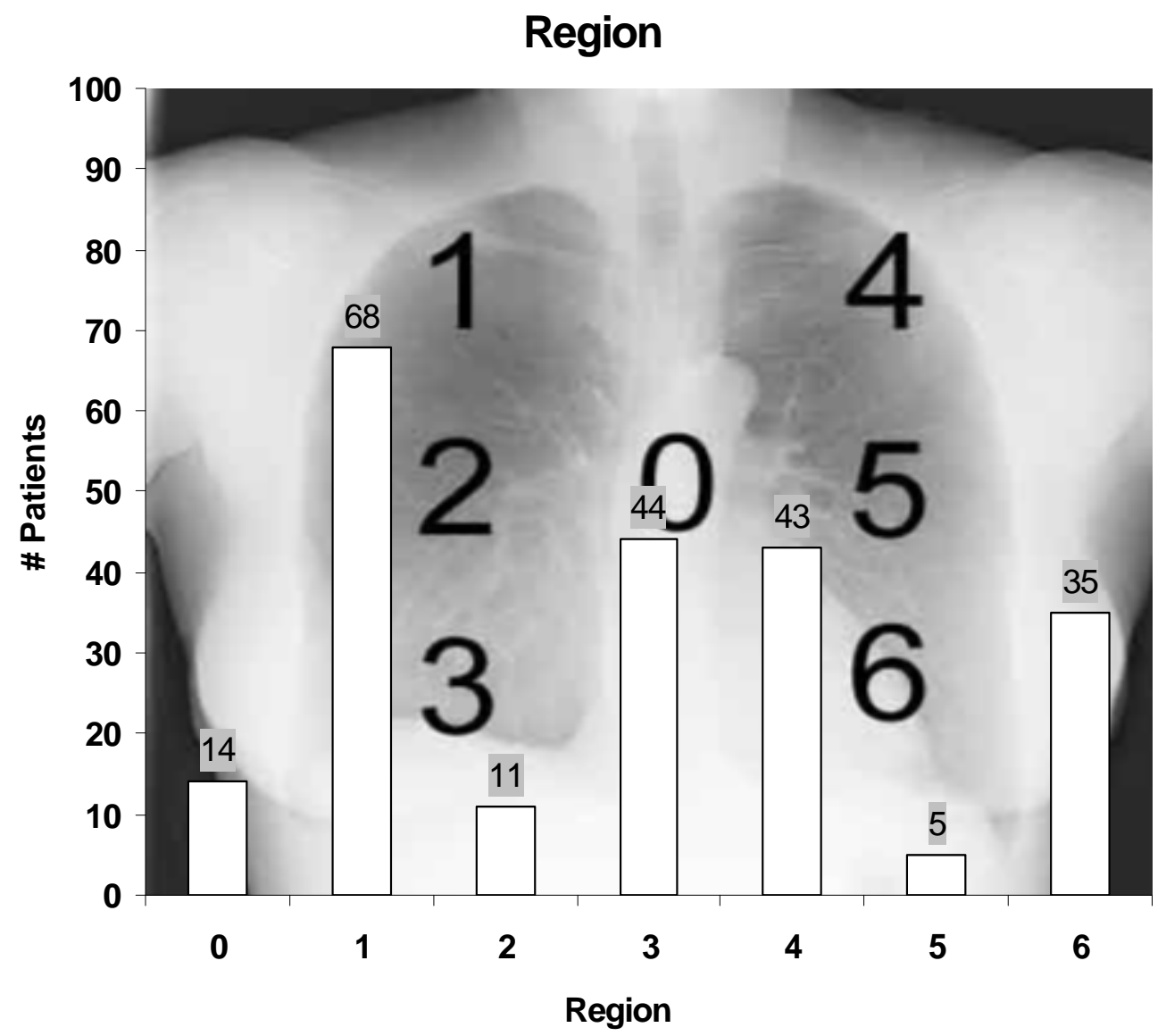

Figure 2.5 A histogram showing the anatomical location of the lung nodule undergoing biopsy. As shown in the histogram, the most frequent location was the right upper lobe. Anatomical lobes of lungs: 1. Right Upper Lobe=RUL, 2. Right Middle Lobe=RML, 3. Right Lower Lobe=RLL, 4. Left Upper Lobe=LUL, 5. Left Middle Lobe= Lingula, 6. Left lower Lobe=LLL, $0=$ Mediastinum. 


\subsubsection{Chest Thickness}

Chest thickness was measured for each patient with two different methods. Firstly, a chest caliper was used to manually measure the anteroposterior (AP) diameter of the patients' thorax before taking the DE projections. Secondly, the skin-skin maximum thoracic AP diameter was measured from the lateral CT scout projection using electronic calipers available on the Picture Archiving and Computer System (PACS). The measurement was made at the level of the T9 vertebra. These measurements were plotted and the resulting histograms are shown in Figure 2.6, with the majority of patients having a thoracic AP diameter $24-26 \mathrm{~cm}$.

Chest Thickness Calipers

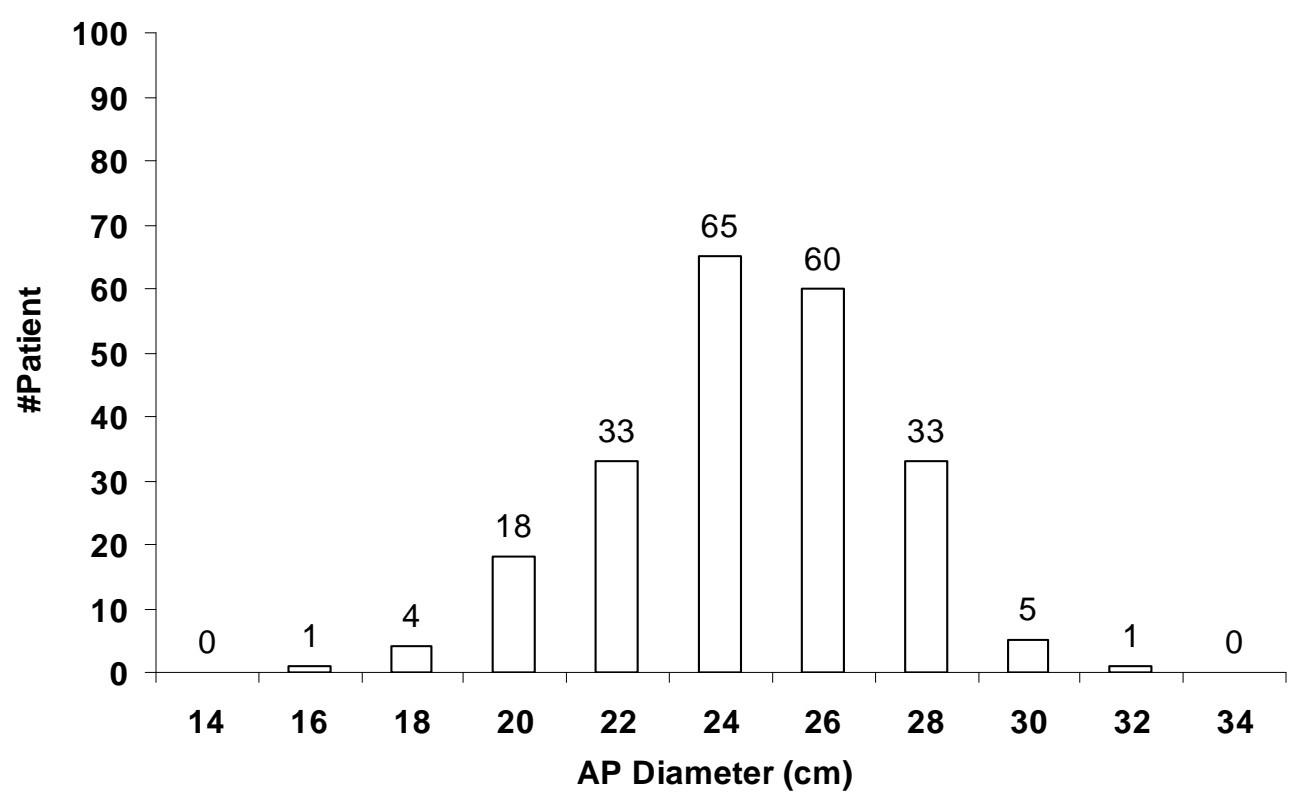



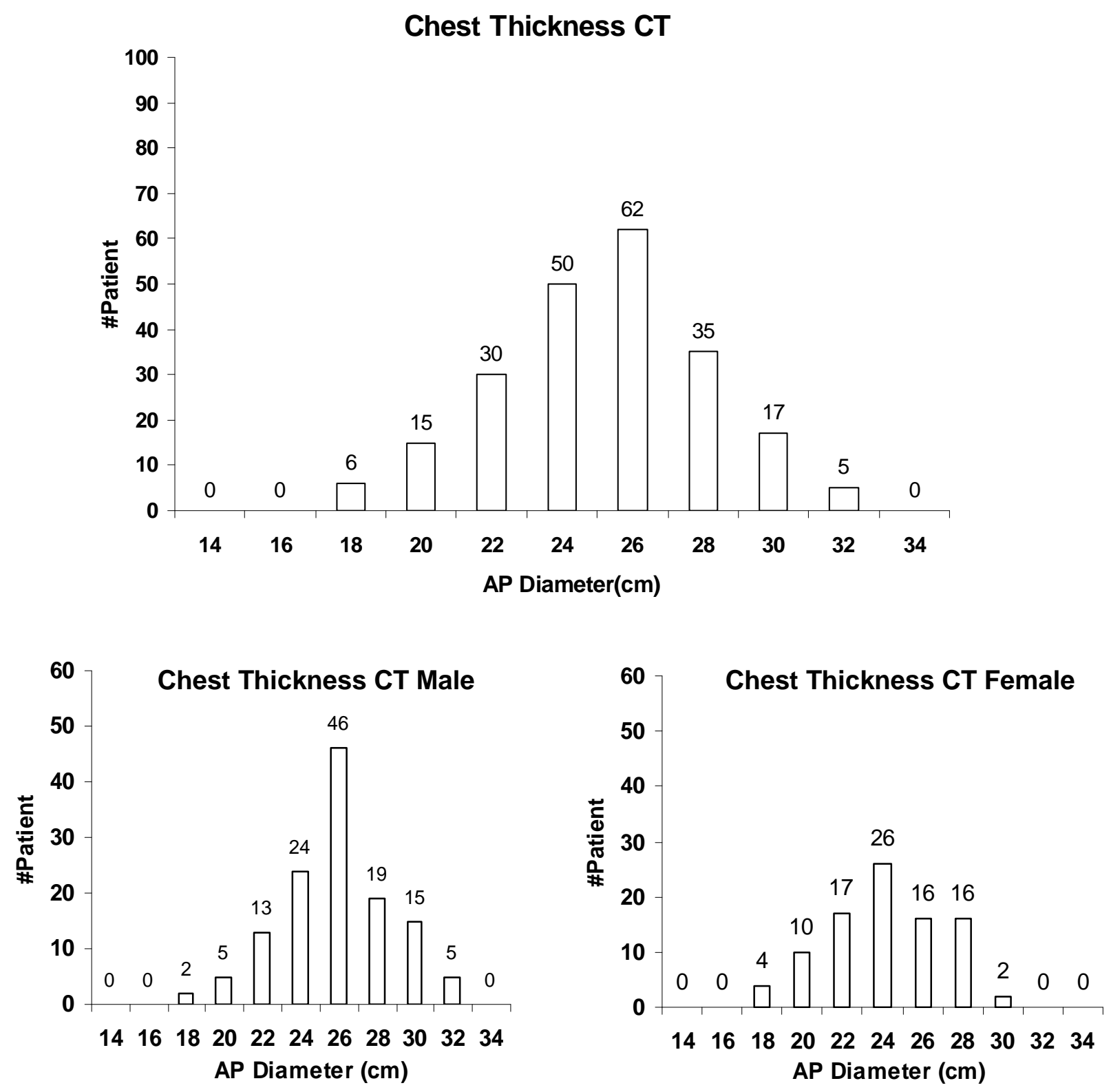

Figure 2.6 Histograms of chest measurements versus frequency in total patient and in each gender individually. The majority of cases have the anteroposterior diameter of the average size patient, being $24-26 \mathrm{~cm}$.

Figure 2.7 below demonstrates the association between the two different methods of AP measurements using regression analysis and measuring the R-squared value. With an R-square 
value of 0.73 , the association is quite strong. This analysis was performed to correlate the accuracy of manual and electronic chest AP diameter measurement.

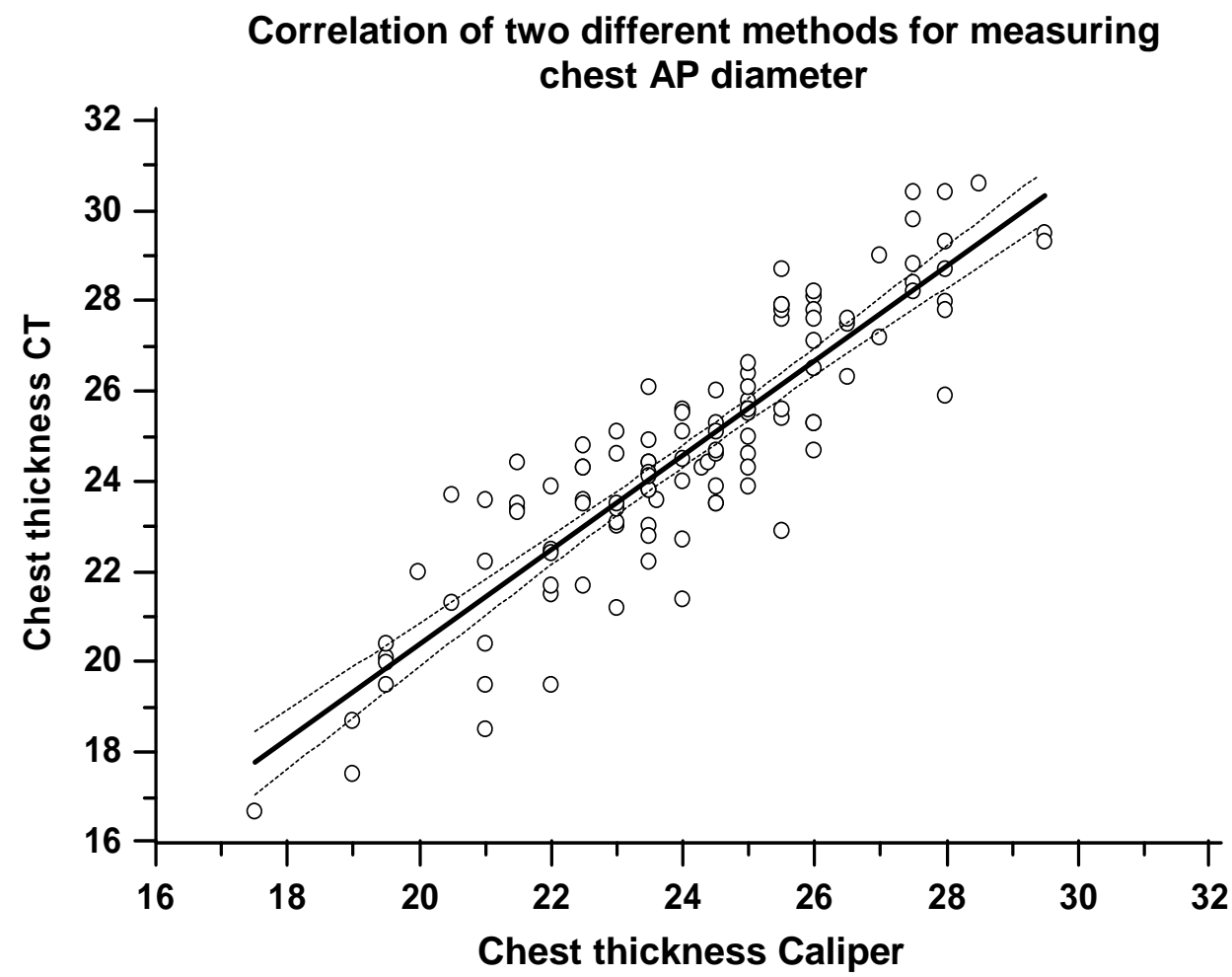

Coefficient of determination $\mathbf{R}^{2}=\mathbf{0 . 7 9 3 0}$ $y=-0.5287+1.0461 x$

\begin{tabular}{llllll}
\hline \hline Parameter & Coefficient & Std. Error & $\mathbf{9 5 \%} \mathbf{C l}$ & $\mathbf{t}$ & $\mathbf{P}$ \\
\hline Intercept & -0.5287 & 1.2236 & -2.9531 to 1.8956 & -0.4321 & 0.6665 \\
Slope & 1.0461 & 0.05051 & 0.9460 to 1.1462 & 20.7120 & $<0.0001$ \\
\hline \hline
\end{tabular}

Figure 2.7 Regression analysis for measuring the anteroposterior diameter on the CT axial projection and by using a manual caliper. R-squared is quite high, showing the strong relationship between the two variables.

As seen in the scatter plot above, measured chest diameters using the two different methods are highly correlated. $(\mathrm{R}=0.79)$ 


\subsection{Disease Characteristics}

\subsubsection{Density}

The density (Hounsfield unit, HU) of each lung nodule was measured using an electronic measuring tool on the PACS workstation (Fusion E-film 2.1; Merge Healthcare, Milwaukee, WI) in By convention, all lesions measuring $\geq 20 \mathrm{HU}$ are considered solid, and any lesion $<20 \mathrm{HU}$ is considered non-solid. The histogram in Figure 2.8 demonstrates the frequency of nodule density measured from the diagnostic thoracic CT scan, performed using $120 \mathrm{kVp}, 50-200 \mathrm{mAs}$ and reconstructed with $5 \mathrm{~mm}$ slice thickness using 50\% overlap. The mean density was $12 \mathrm{HU}$ with a range of -592 to $180 \mathrm{HU}$. All the measurements were performed using the standard dose thoracic $\mathrm{CT}$, performed on a 64MDCT slice scanner, with $120 \mathrm{kVp}$, modulated tube current, with $5 \mathrm{~mm}$ reconstructed slice thickness.

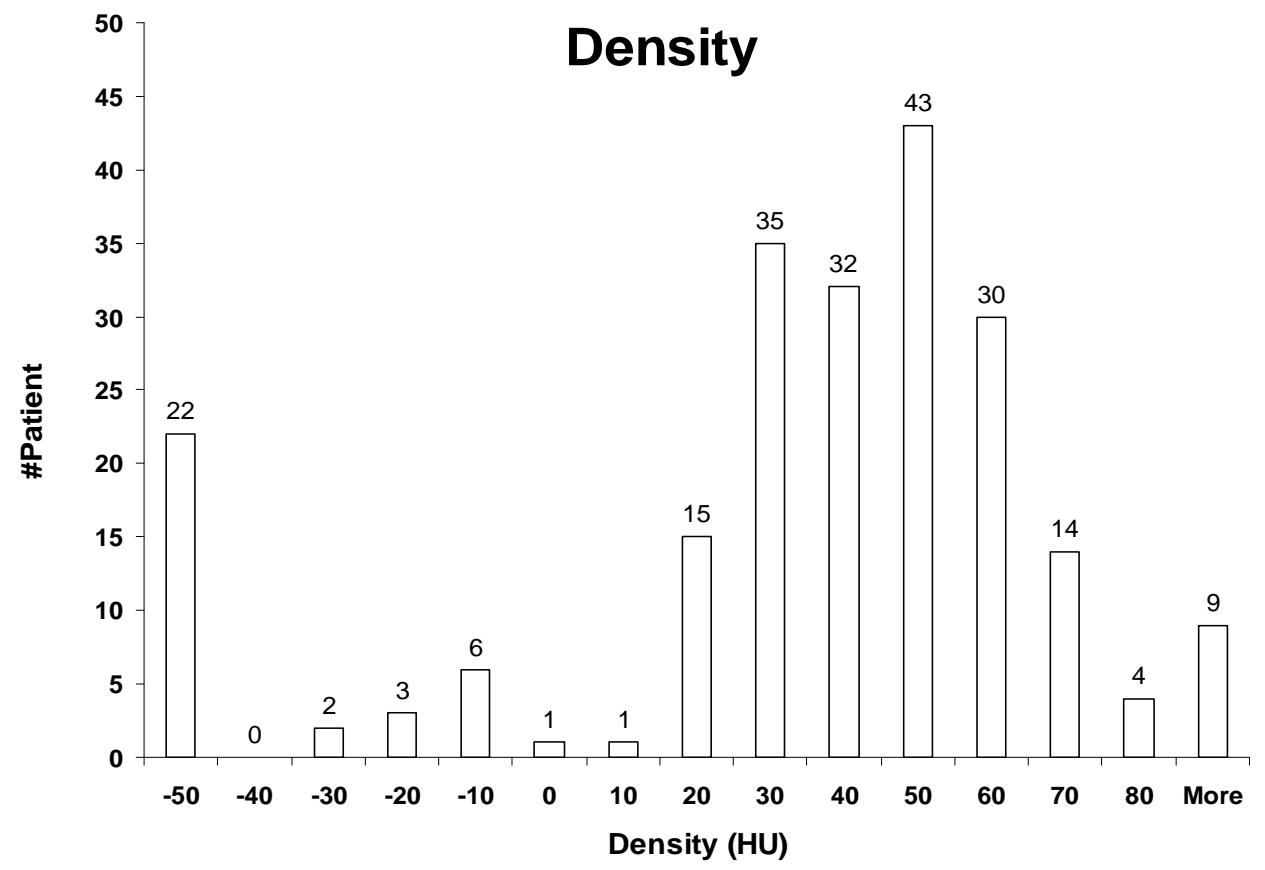

Figure 2.8 A histogram showing the number of cases in each density group. 


\subsubsection{Size}

The diameter of each nodule was measured using electronic calipers on the axial CT projection. The largest diameter of the lesion in the cross-sectional image, displayed on a wide window and level setting (W1600 L -600) was measured and recorded. Figure 2.9 demonstrates a histogram of the frequency of lung nodules in each size range. The average size was $2.7 \mathrm{~cm}$ and sizes ranged from 0.7 to $13.9 \mathrm{~cm}$.

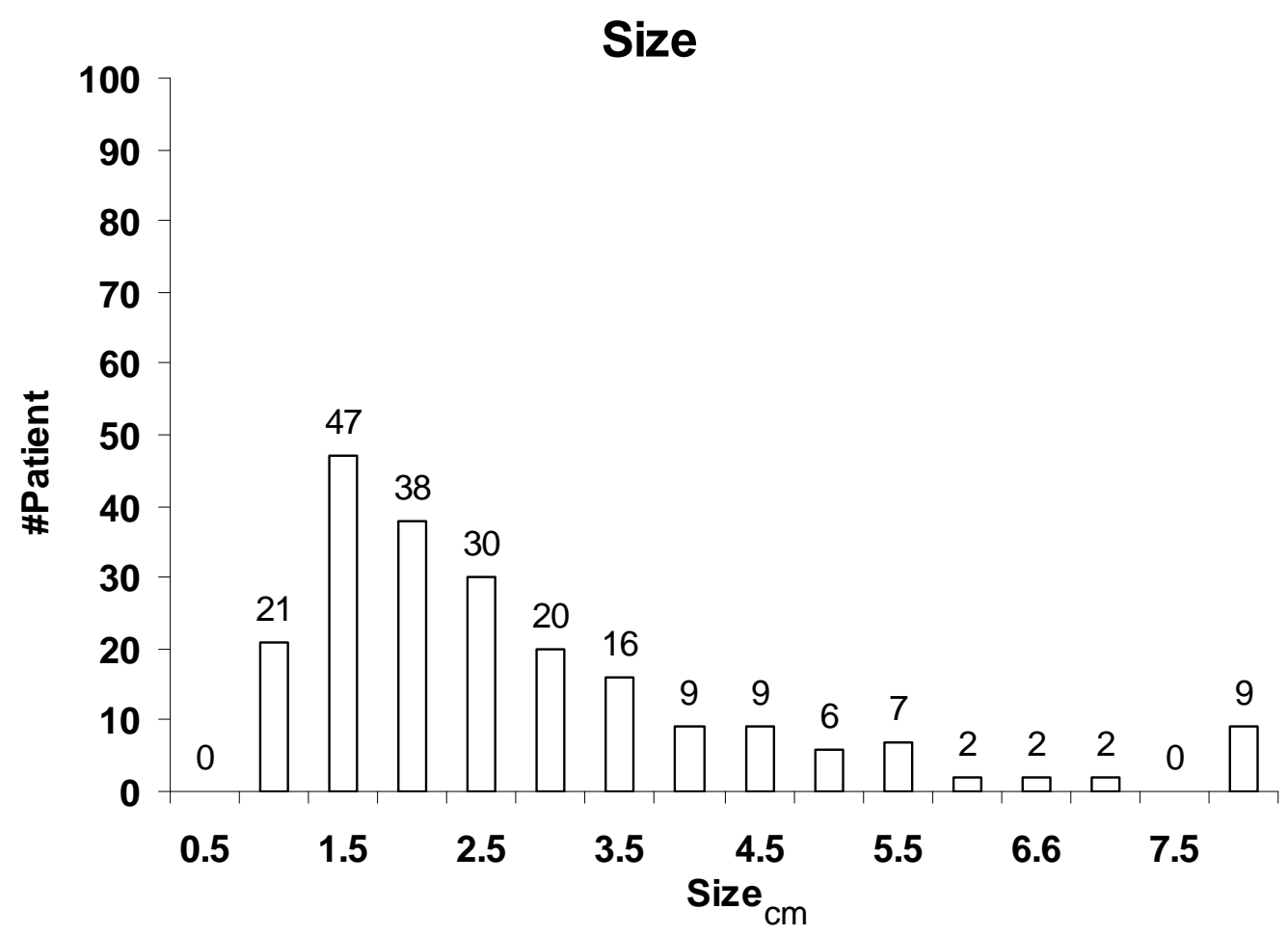

Figure 2.9 A histogram showing the number of cases in each nodule size group.

\subsubsection{Pathology}

Percutaneous CT-guided transthoracic biopsies were performed immediately following the DE/DR imaging exam to provide a definitive diagnosis of the lung lesion. Biopsies were performed either by fine-needle aspiration and cytologic examination or by core biopsy and 
histologic examination. The principal lung nodule pathology was non small cell lung cancer (Figure 2.10).

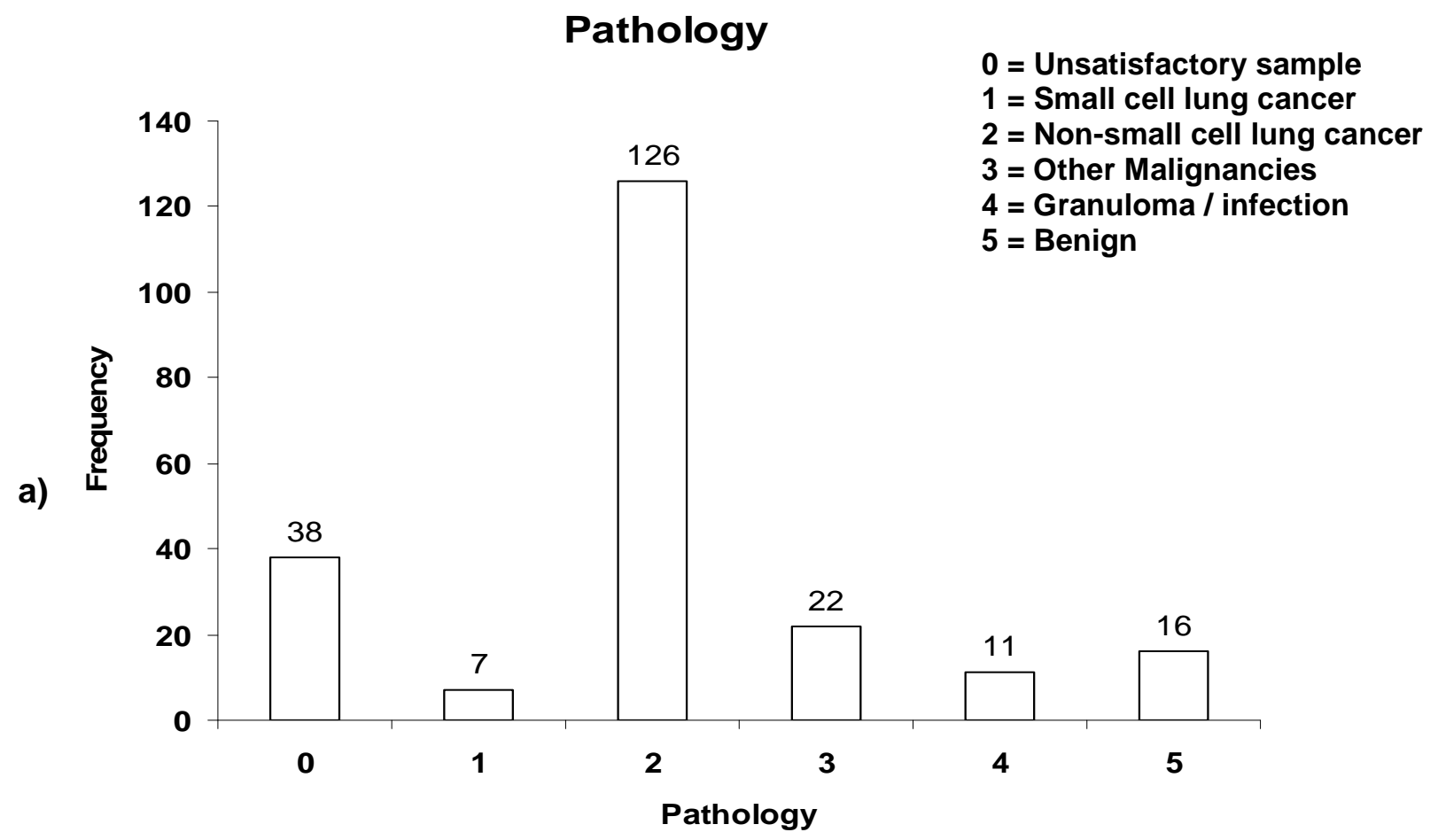

Figure 2.10 Illustration of lung nodule pathology. The most common pathology was non-small cell carcinoma and the most common type of non-small cell carcinoma was adenocarcinoma.

The most common type of pathology was non-small cell carcinoma (NSCC), with adenocarcinoma representing the most frequent subtypes of NSCC.

\subsection{Exam Characteristics}

\subsubsection{Repeat Breath Hold}

Patients were instructed not to breathe during the exams between the low- and high-energy projections, but in 14/220 patients $(6 \%)$ technical issues created longer than anticipated delay 
times (6-8s) between the two projections, and therefore there was a second breath hold.

Occasionally breathing artifacts were seen in the resultant images.

\subsubsection{Time between the Exposures}

As mentioned above, a 6-8-second delay usually occurred between the two projections due to detector acquisition delay. The delay time was different from cases to case and in some cases was longer due to technical difficulties. To determine how many cases had more than the usual delay, the frequency of cases in each delay time interval is plotted in Figure 2.11.

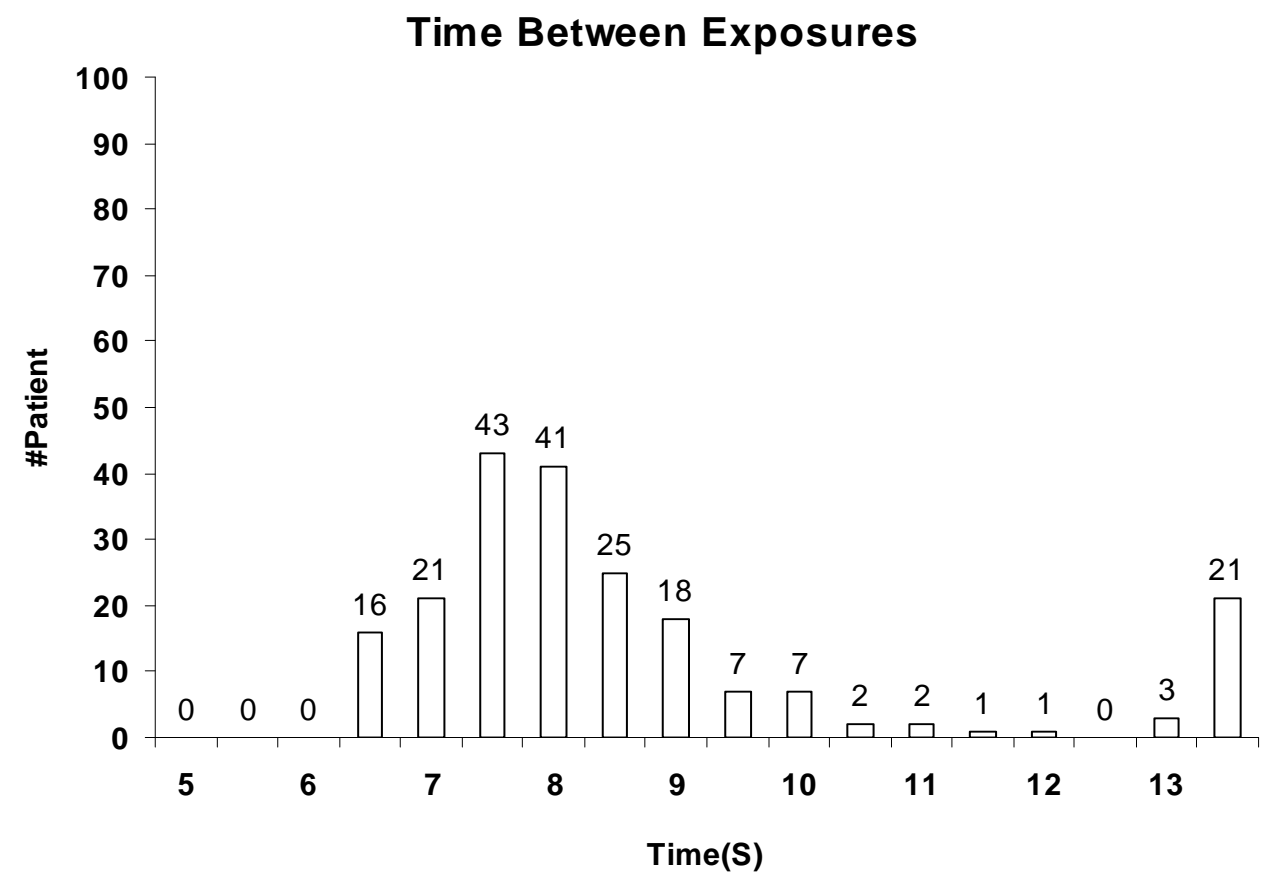

Figure 2.11 Projections acquisitions frequency for different time delay intervals. As observed in the histogram above, most of the studies were performed with an average delay of 6-9

\subsubsection{Heart Rate}

Patients' heart rates (HR) varied during low- and high-energy projections. Figure 2.12 below shows patients' HR variations in high- and low-energy projections. 

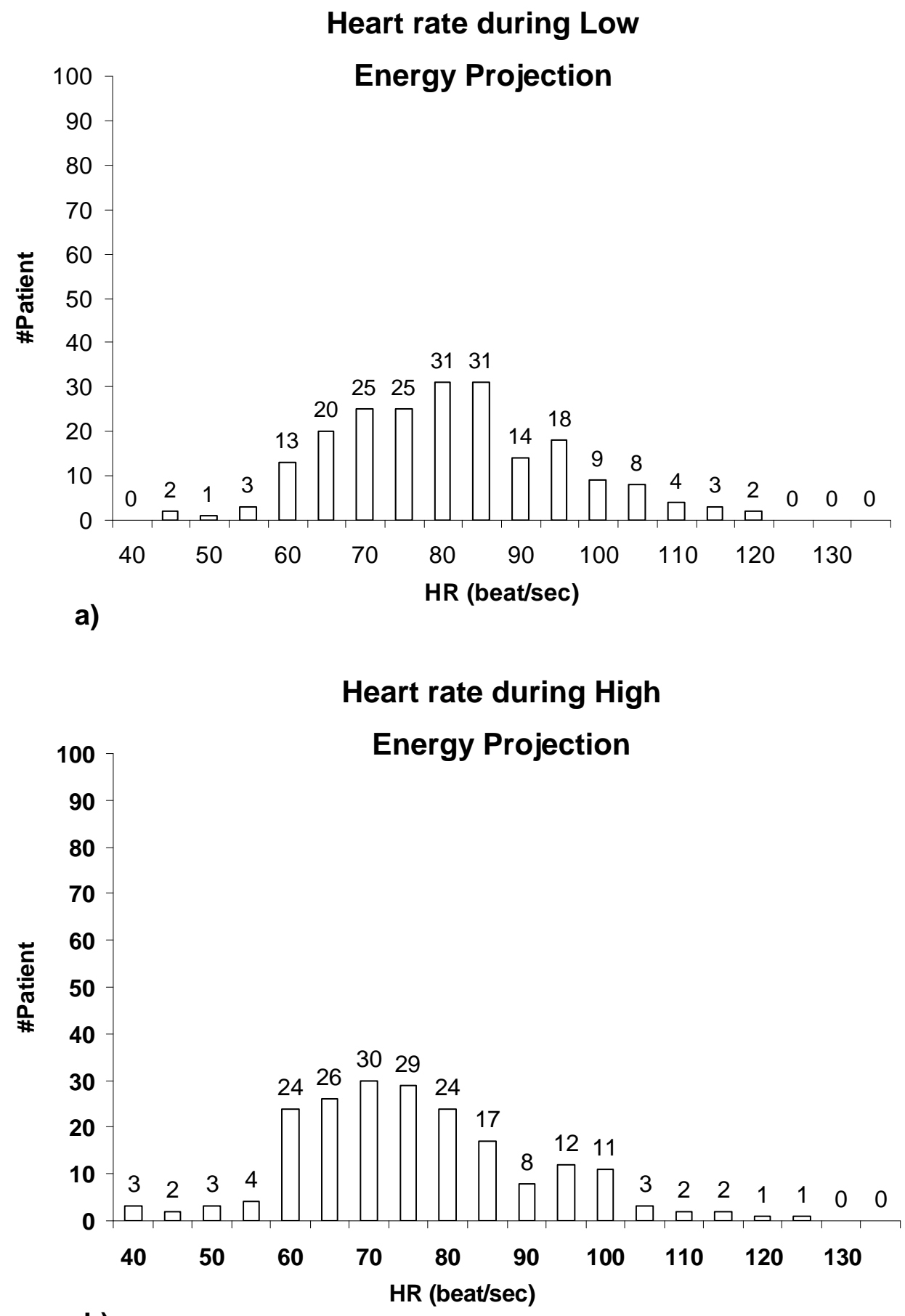

b)

Figure 2.12 HR variation during high- and low-energy projections. As shown here, most of the patients had a HR of about $60-80$ (beat/sec) during projections. But there is a shift towards a lower rate during the high-energy projection, the second acquisition. This could be explained by 
the effect of resulting hypoxemia on the chemo receptors of the carotid body and the following vagal stimulation.

Figure 2.16 below shows the association between HR during low and high projection acquisitions.

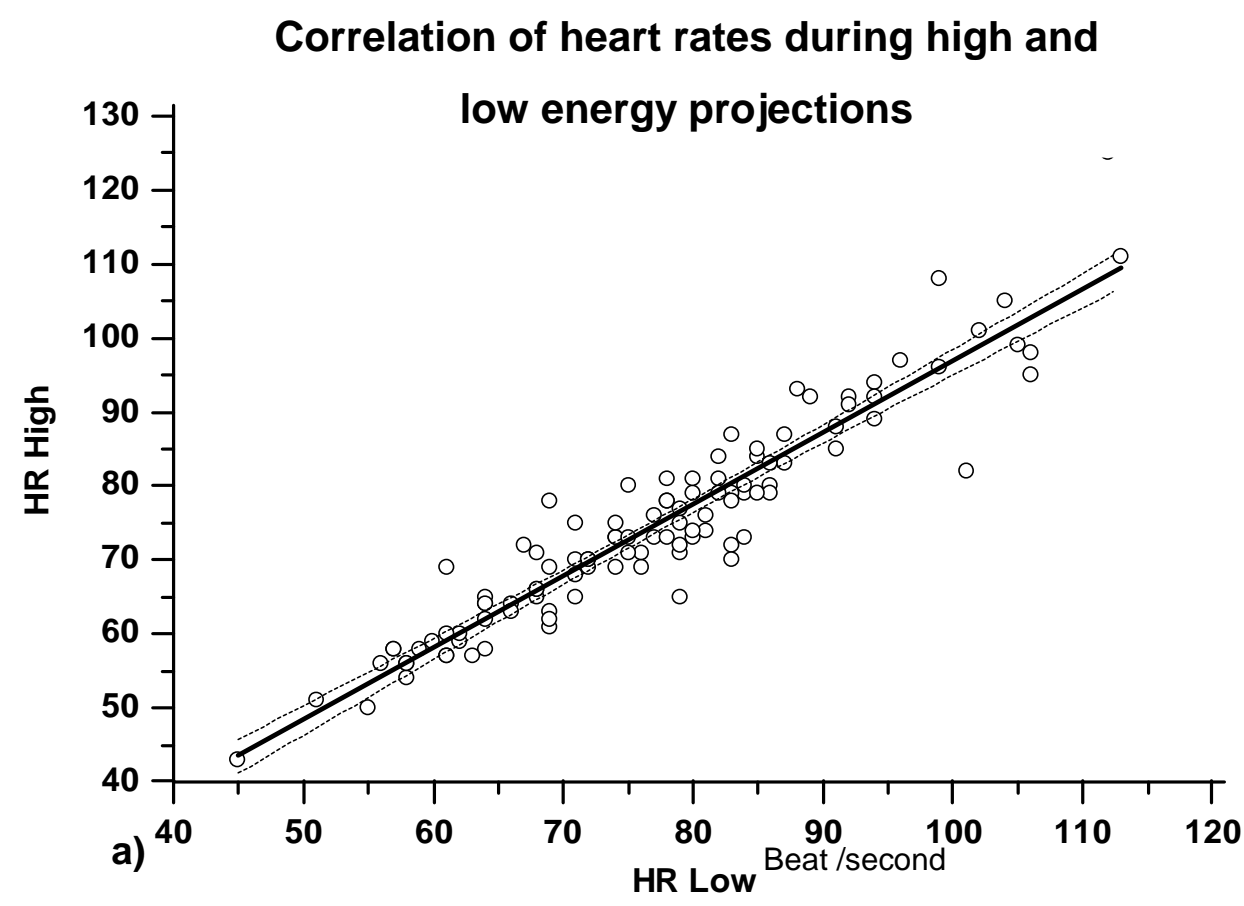

Coefficient of determination $R^{2}=0.8931 \quad y=-0.004085+0.9684 x$

\begin{tabular}{lrrrrr}
\hline \hline Parameter & Coefficient & Std. Error & \multicolumn{1}{c}{ 95\% Cl } & \multicolumn{1}{c}{ t } & \multicolumn{1}{c}{$\mathbf{P}$} \\
\hline Intercept & -0.004085 & 2.5647 & -5.0894 to 5.0812 & -0.001593 & 0.9987 \\
Slope & 0.9684 & 0.03269 & 0.9035 to 1.0332 & 29.6233 & $<0.0001$ \\
\hline \hline
\end{tabular}

Figure 2.13 Regression analysis. The coefficient of determination for heart rate during low- and high-energy projections. The HRs are highly correlated $(\mathrm{R} 2=0.69)$. 


\subsection{DE Image Characteristics}

\subsubsection{Tissue Cancellation Parameters: Ws, Wb, Wc, Wn}

Tissue cancellation parameters were selected manually during image decomposition in consensus with an observer who was trained by an experienced thoracic radiologist regarding the optimal presentation of the important anatomical landmarks. Future work will include the use of more detailed, patient-specific, automated and spatially varying parameter selection and will consider incorporation of $w_{s}$ and $w_{b}$ as parameters that may be freely varied by the radiologist in a manner analogous to varying the display window/level.

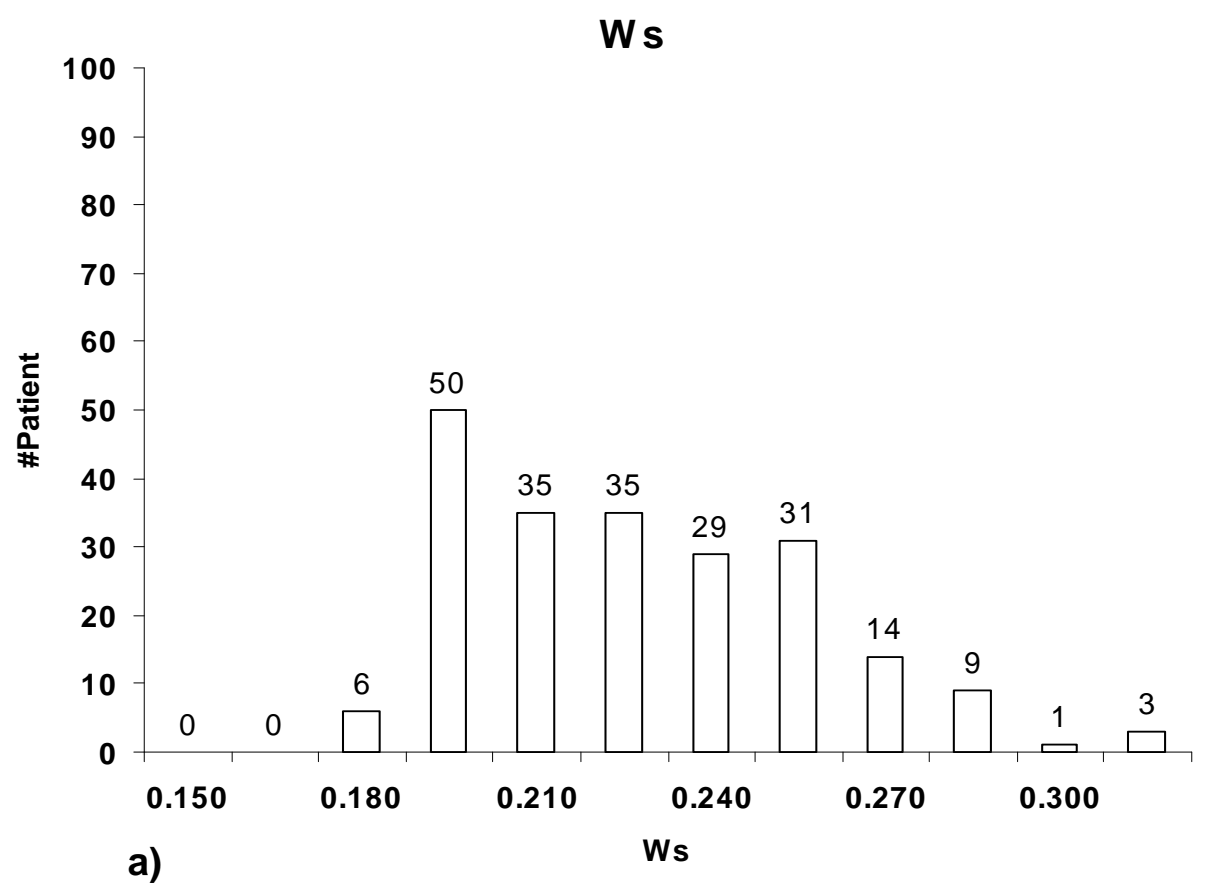




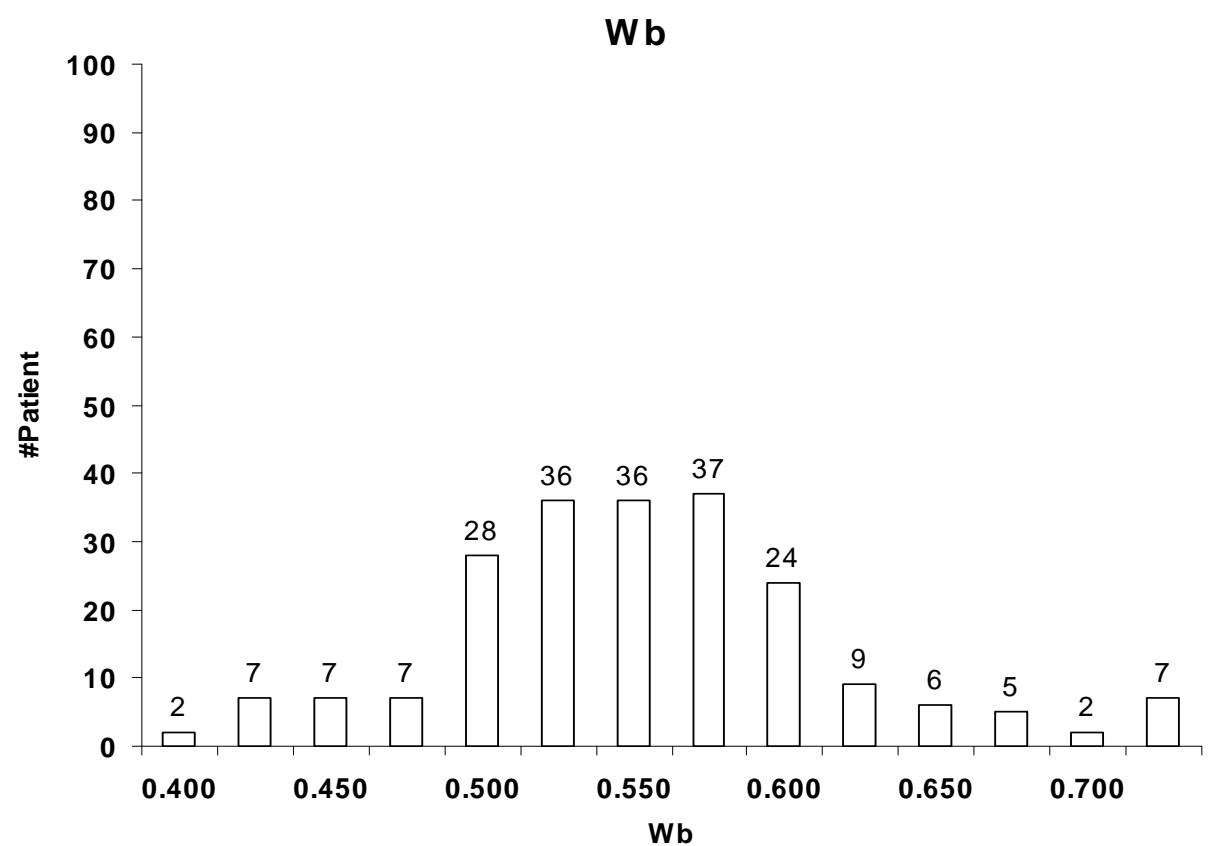

b)

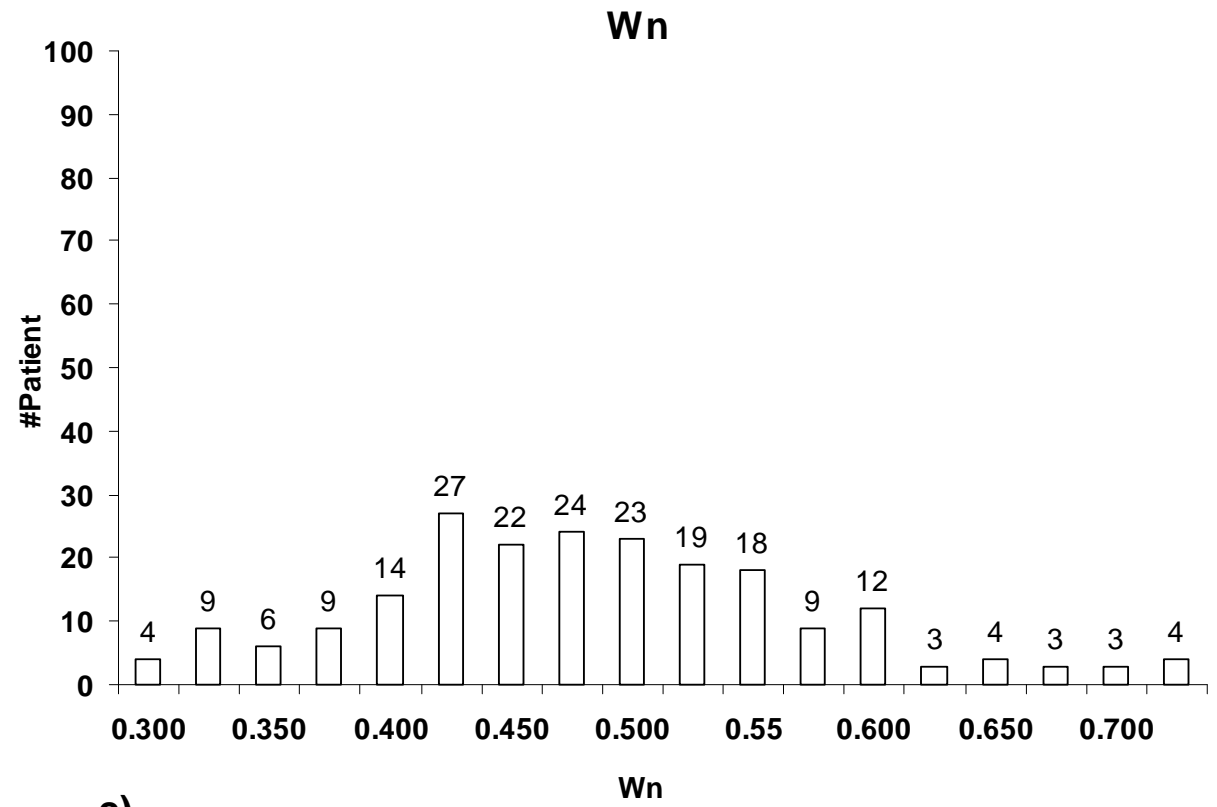

c) 


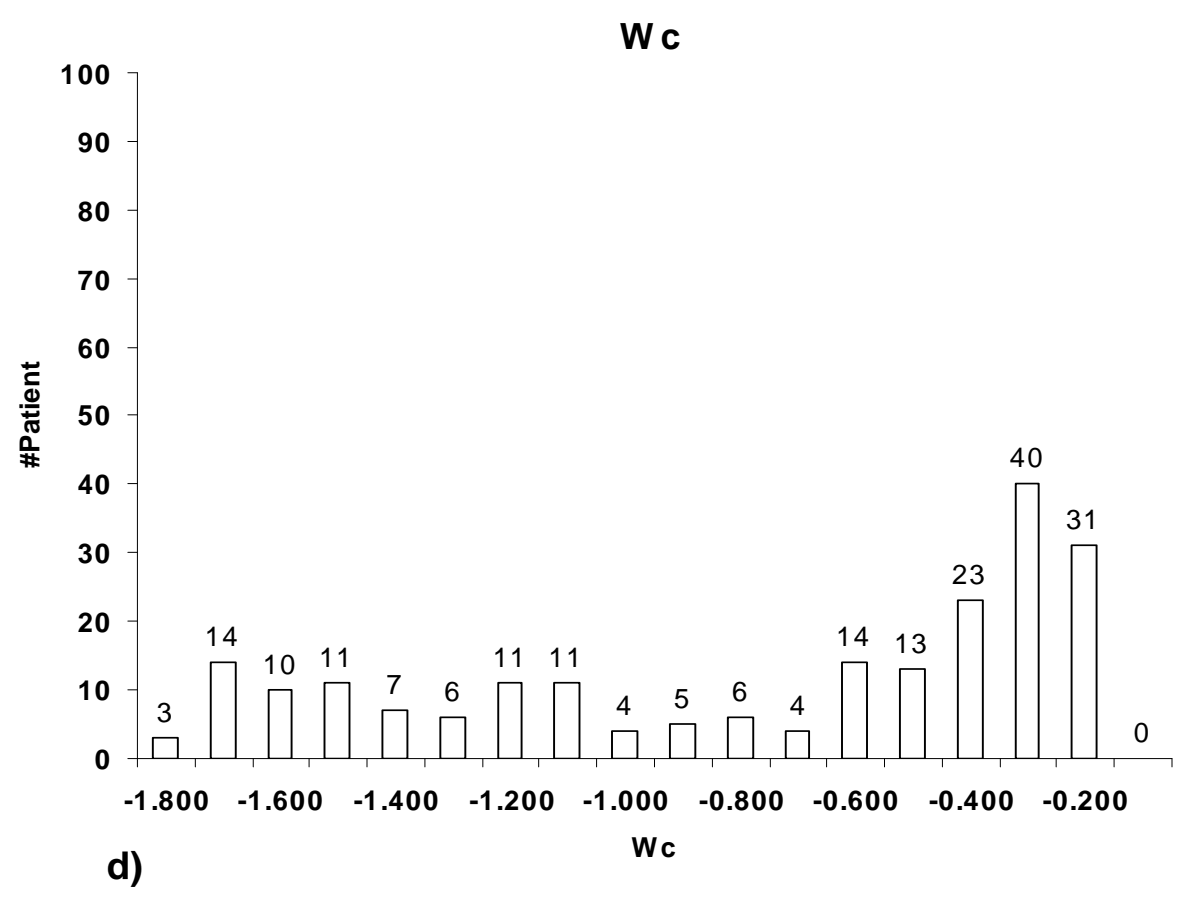

Figure 2.14 (a, b, c, d) Frequency of cases in different Weighting factor (W) ranges for different decompositions.

\subsection{Multi Scale Multi Resolution (MSMR) Registration Algorithm}

As mentioned previously, occasional delay between two dual energy projections acquisition and the nature of dual energy techniques (log-weighted subtraction) causes image quality degradation in the resulting dual energy images. This is mainly due to different type of motion artifact including breathing, cardiac and patient motion. Different techniques were used before acquiring the projections to reduce these artifacts as much as possible. These included, instructing patients to hold their breath and hold still, during the high- and low-energy projection acquisitions, and also using cardiac gating techniques (pulse oximeter). For residual problems, registration techniques were used to maximize the coordination of the two images. The second projection (high-energy image) was aligned (registered) onto the low-energy image by means of a deformable registration technique. The registration algorithm operated $\mathrm{n}$ multiple passes at 
progressively smaller scales and increasing resolution (57).Different variables of the registration technique are discussed below.

\subsubsection{Mean Vector displacement (MVD) and Standard Deviation Displacement (SDD)}

As mentioned above the registration algorithm operates on multiple scales and at multiple resolutions to transform the $\mathrm{HE}$ image. A total of four iterative passes were used in the reported registration technique herein where in each pass a series of translation vectors was calculated. A spatial transformation inferred from these vectors, was interpolated and applied to the high energy image in a pixel-wise manner. The transformed HE image and the original LE image then made the input for the next pass where the process was repeated. The mean vector displacement (MVD, mm) for each case was calculated. To do so, for each single ROI, overall X and $\mathrm{Y}$ coordinates displacement at the end of final pass were measured the total vector displacement was calculated by means of Pythagorean Theorem: $V D=\sqrt{X^{2}+Y^{2}}$ where VD is the vector displacement and $\mathrm{X}$ and $\mathrm{Y}$ the overall coordinates displacement.

To calculate the mean vector displacement (MVD) for each single case, all vector displacements for all ROIs (mm) were averaged, over the number of ROIs. The MVD and standard deviation (SD) for each case were plotted against the number of cases in each range as shown below. 

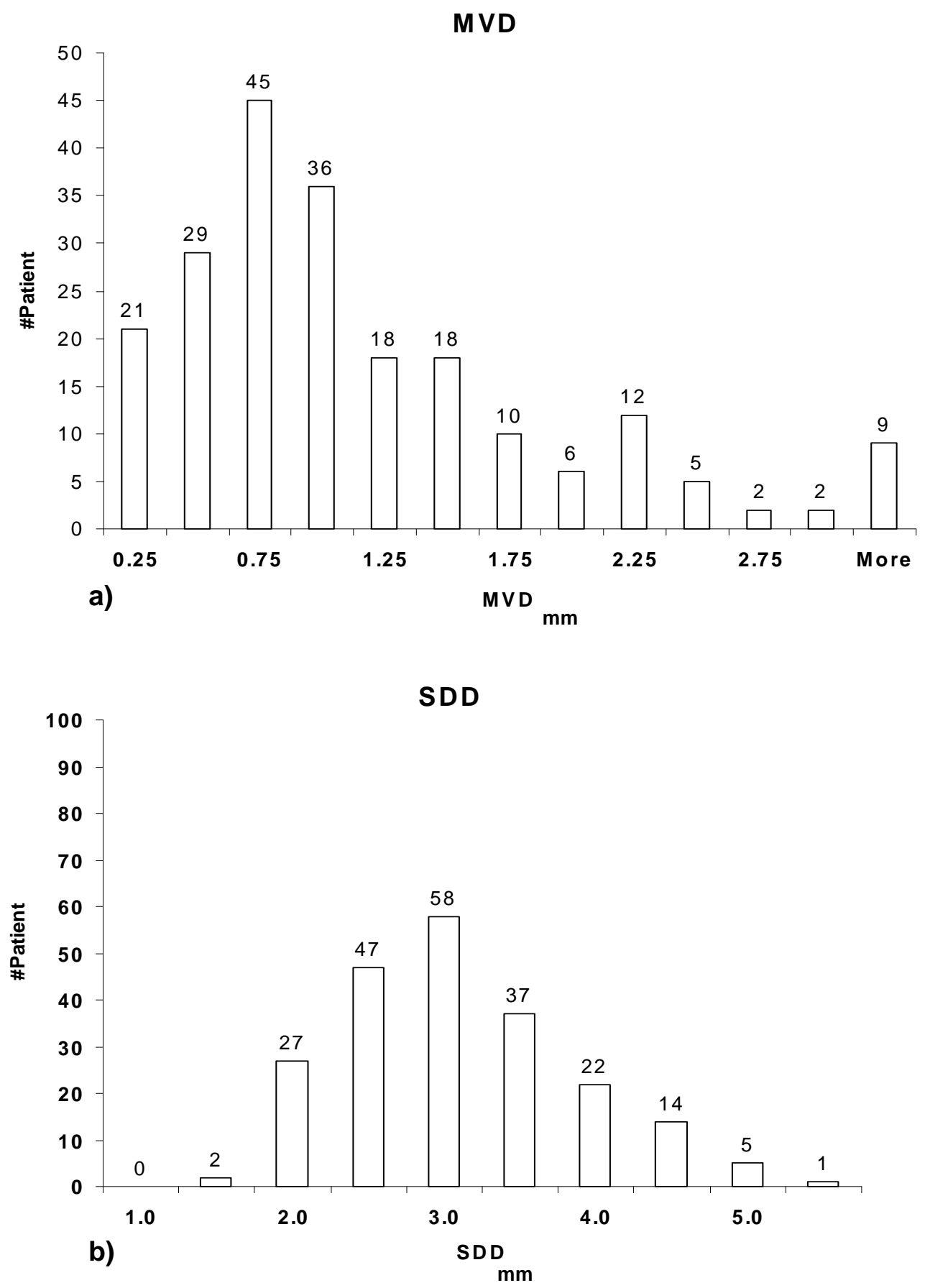

Figure 2.15 (a, b) Histograms showing frequency of cases in different ranges of MVD and SD of MVD. 


\section{Chapter 3 Satisfaction Test}

\section{Observer Study}

For the observer study described below, the DE images were presented as a "two-slice volume" that could be viewed by mouse wheel scrolling between the soft-tissue and bone images. Five expert observers (3 radiology fellows, 2 radiology staff, each a specialist in chest imaging) were independently presented with the images, one at a time in a randomized order, and asked to rate each image according to the 9-point diagnostic satisfaction scale shown in Table. 3.1

\begin{tabular}{|c|c|c|}
\hline Score & Rating & Description \\
\hline 9 & Very satisfied & $\begin{array}{c}\text { The abnormality is perfectly obvious and easily } \\
\text { characterized. }\end{array}$ \\
\hline \multicolumn{3}{|l|}{8} \\
\hline 7 & Satisfied & $\begin{array}{l}\text { The abnormality is visible and can be well } \\
\text { characterized. }\end{array}$ \\
\hline \multicolumn{3}{|l|}{6} \\
\hline 5 & $\begin{array}{l}\text { Neither satisfied nor } \\
\text { dissatisfied }\end{array}$ & $\begin{array}{l}\text { The abnormality is reasonably well seen and } \\
\text { characterized. }\end{array}$ \\
\hline \multicolumn{3}{|l|}{4} \\
\hline 3 & Dissatisfied & $\begin{array}{c}\text { The abnormality is visible, but detection and } \\
\text { characterization of subtle features are a bit } \\
\text { challenging. }\end{array}$ \\
\hline \multicolumn{3}{|l|}{2} \\
\hline 1 & Very dissatisfied & $\begin{array}{l}\text { The abnormality could be overlooked or } \\
\text { mischaracterized. }\end{array}$ \\
\hline
\end{tabular}

Table 3.1 Satisfaction rating scale. This table was displayed to observers on a second monitor during all tests as shown in table 3.1. 
Scales with fewer (5) and more (up to 100) points were considered in preliminary studies, with 9 found to be tolerated well by observers and consistent within reasonably fine levels of image quality discrimination, as described by Van Metter and Foos. (58) The scale was visible to observers at all times on a second monitor (illustrated in Fig. 2.1).

The satisfaction or preference test performed here is essentially a subjective rank-order evaluation. This method is usually used for answering questions that pertain to different image processing (rendering) variations where the choices tend to be heavily weighted in terms of observer preference, and where detection performance differences is not necessarily expected. (58) An advantage of the approach is that the assessment of overall impression allows for a greater variety of image types to be included more easily into the study, and consequently the results from the study tend to be robust in that can be applied to an extended population of images. This is because the reader response provides an overall impression of the interpretability of the image, and is not just focused for any single specific task. A disadvantage of this approach is that it does not provide objective performance results as there is no "truth" associated with the image that the response is compared against, so the results in a sense are softer. The proponents of this methodology advocate this approach as it has generated consistent results (responses) among readers in their studies. (59) However, a wider validation of this approach has not been performed.

Preceding each observer test, a training session involving 8 DE and 7 DR images was conducted to familiarize the observers with the software and standardize their understanding of the rating scale. The training images were drawn from the pool of cases available at the time of the study and did not overlap with those used as test images. To examine intra-reader consistency during the actual test, the first 7 images displayed in each test were displayed again at the end of the test 
(without informing the observers that the images were repeated), with differences examined in terms of the Wilcoxon signed rank p-value, as described below. The first 7 images were rejected from the study, except for purposes of intra-reader variability; therefore, the analysis pertains to each DE and DR image shown only once (no repeats). Repeat readings were found to be highly reproducible for expert observers in preliminary studies $(\mathrm{p}<0.001$, Wilcoxon signed rank test) and would not add to the statistical power of the study. The study was conducted in a clinical radiology reporting room with subdued lighting on diagnostic-quality; monochrome LCD monitors (AM-QX21-A9300, National Display, San Jose, CA)

\section{Statistical Analysis}

\subsection{Fraction of Responses at or above a Given Rating}

The rating scale responses constitute ordinal data. The fraction of observer responses $(\mathrm{F})$ at or above a given rating $(\mathrm{R})$ was plotted versus the rating scale, giving curves that range 0 to 1 on the vertical axis plotted versus the ordinal rating scale (1 to 9 ) on the horizontal axis - essentially a cumulative histogram of responses. The fraction at a rating of 1 is 1 by definition. A higher curve for a given modality indicates a greater fraction of higher ratings (a greater degree of diagnostic satisfaction) for that modality.

Error bars on such plots reflect a two-sided $95 \%$ confidence interval computed according to a binomial distribution as described below. The corresponding error bars are asymmetric and appropriately bounded between 0 and 1 . Each image was scored as either (i) at or above a certain rating, or (ii) below that rating, giving two mutually exclusive outcomes such that $F$ (the 'fraction at or above a given rating') follows a binomial distribution. The upper and lower bounds of the confidence intervals were calculated as: 


$$
\begin{aligned}
& \text { Upper bound: } \sum_{\kappa=0}^{N_{d}}\left(\begin{array}{l}
N \\
\kappa
\end{array}\right) p_{U}^{\kappa}\left(1-p_{U}\right)^{N-\kappa}=\frac{\alpha}{2} \\
& \text { Lower bound: } \sum_{\kappa=0}^{N_{d}-1}\left(\begin{array}{l}
N \\
\kappa
\end{array}\right) p_{L}^{\kappa}\left(1-p_{L}\right)^{N-\kappa}=\frac{1-\alpha}{2}
\end{aligned}
$$

Where $N$ is the total sample size, $N_{d}$ is the number of samples with the outcome of interest, $a$ is the level of significance (5\%), $p$ is the proportion of samples with the outcome of interest, $p_{U}$ is the upper bound of $p$, and $p_{L}$ is the lower bound of $p$. The upper and lower bounds were calculated using Newton-Raphson method in Matlab (The Mathworks, Natick MA) to a precision of $1 \times 10^{-6}$.

\subsection{Statistical Significance: P-value from Wilcoxon Signed Rank Test}

The statistical significance in differences observed between DE and DR scores was evaluated in terms of the p-value at a 95\% level of significance from a Wilcoxon signed rank test - a nonparametric test suitable to paired ordinal data, assuming all observations within a given modality are independent. (60) The p-value was calculated using the Matlab function 'sign rank' accounting for both the sign and magnitude of the difference in ratings

To examine intra-reader consistency, the Wilcoxon signed rank test was also used in relation to the first and last 7 images in the reading study, repeated at the beginning and end of the test as described above. In this case, the alternative hypothesis was that 'the two sets of scores are not equivalent;' therefore, a two-sided p-value was calculated directly from 'sign rank'. Across 5 observers, all p-values assessing intra-reader consistency were greater than 0.05 (specifically, pvalue $=0.125,1,0.0625,0.5313$, and 1 for observers A-E, respectively), suggesting that there was no significant difference in observer readings at the beginning and end of the test. 


\subsection{Stratification of the Results}

Performance was analyzed for all cases pooled, as well as by post-hoc stratification of the data according to lesion size, lesion density, chest thickness, gender, and location of the lesion. Lesion size was characterized as the greatest linear dimension as measured on CT, and the results were stratified as "nodule" $(\leq 3 \mathrm{~cm})$ or "mass" $(>3 \mathrm{~cm})$, consistent with typical clinical terminology. Lesion density was measured using the attenuation measurement tool on a PACS workstation (Fusion E-film 2.1, Merge Healthcare, Milwaukee, WI), and the results were stratified as "Solid" ( $\geq 20 \mathrm{HU})$ or "Non-solid" (<20 HU). Chest thickness was characterized as the anterior-posterior distance measured from the xiphoid process to the T9 thoracic vertebra taken from the axial CT image at this level, and the results were stratified as "Average" $(\leq 26 \mathrm{~cm})$ or "Thick" (>26 cm). Lesion location was determined according to the anatomical position with respect to lung zones (or mediastinum), and the results were stratified as "Right-Upper," "LeftUpper," "Right-Middle," "Left-Middle," Right-Lower," "Left-Lower," and "Mediastinum." In terms of stratification analysis, since multiple comparisons (five) were performed on the same set of data, the threshold level for a significant $\mathrm{p}$ value was considered to be less than $0.01(0.05 / 5)$. (61) The number of cases overall and within each stratum is summarized in Table 2.1 


\begin{tabular}{|c|c|c|c|c|}
\hline Stratification & Description & $\begin{array}{c}\text { Number of } \\
\text { cases }\end{array}$ & $\begin{array}{l}\text { Number of } \\
\text { readings }\end{array}$ & P Value \\
\hline All pooled & & 55 & 275 & $<.001$ \\
\hline \multirow[t]{2}{*}{ Lesion size $(\mathrm{cm})$} & $<3$ & 36 & 180 & $<.001$ \\
\hline & $>3$ & 19 & 95 & .0264 \\
\hline \multirow{2}{*}{$\begin{array}{l}\text { Lesion Density } \\
\text { (Hounsfield units) }\end{array}$} & $<20$ & 9 & 45 & .0968 \\
\hline & $>20$ & 46 & 230 & $<.001$ \\
\hline \multirow[t]{2}{*}{ Chest thickness (cm) } & $<26$ & 47 & 235 & $<.001$ \\
\hline & $>26$ & 8 & 40 & .0015 \\
\hline \multirow[t]{2}{*}{ Gender } & Male & 31 & 155 & $<.001$ \\
\hline & Female & 24 & 120 & .0137 \\
\hline \multirow[t]{7}{*}{ Region } & Right upper & 20 & 100 & .0012 \\
\hline & Left upper & 5 & 25 & .0067 \\
\hline & Right middle & 4 & 20 & .1800 \\
\hline & Left middle & 2 & 10 & .0313 \\
\hline & Right lower & 8 & 40 & .3625 \\
\hline & Left Lower & 9 & 45 & .0010 \\
\hline & Mediastinum & 7 & 35 & .2455 \\
\hline
\end{tabular}

Table 3.2 Summary of Cases and Stratifications of Data. The $P$ values relate to the statistical significance in the difference between satisfaction with dual-energy and digital radiographic images, regarded as significant for $\mathrm{P}<.01$.

\section{Result}

\subsection{All Cases Pooled}

The results for the 275 total ratings ( 5 radiologists $* 55$ cases) for each of the two modalities (DE and DR) are summarized in Fig. 3.1. Individual case-by-case comparison of DE and DR for each patient is evaluated in Fig. 3(a). In 41.5\% (114/275) of cases, the DE image was rated superior to 
DR by at least a difference of $\mathrm{R}=1$. In $38.9 \%$ (107/275) of cases, the DE and DR images were rated equal. In $19.6 \%$ (54/275) of cases, the DR image was rated superior. Further to this caseby-case examination, the proportion of cases for which one modality was superior (or equal) to the other, as judged by 3 or more of the 5 observers, is plotted in Fig. 3.1(b). Of these: $36.4 \%$ (20/55) scored DE superior to DR; 36.4\% (20/55) rated DE and DR equivalent; and 5.5\% (3/55) rated DR superior to DE. In the remaining 12 cases $(21.8 \%)$, a majority could not be reached regarding the superiority / equality / inferiority between the two modalities. The fractions of images rated at or above a given rating score [Fig. 3.1(c)] shows that DE rated consistently higher than DR (p-value $<0.001)$ in the detection and characterization of lung nodules. 

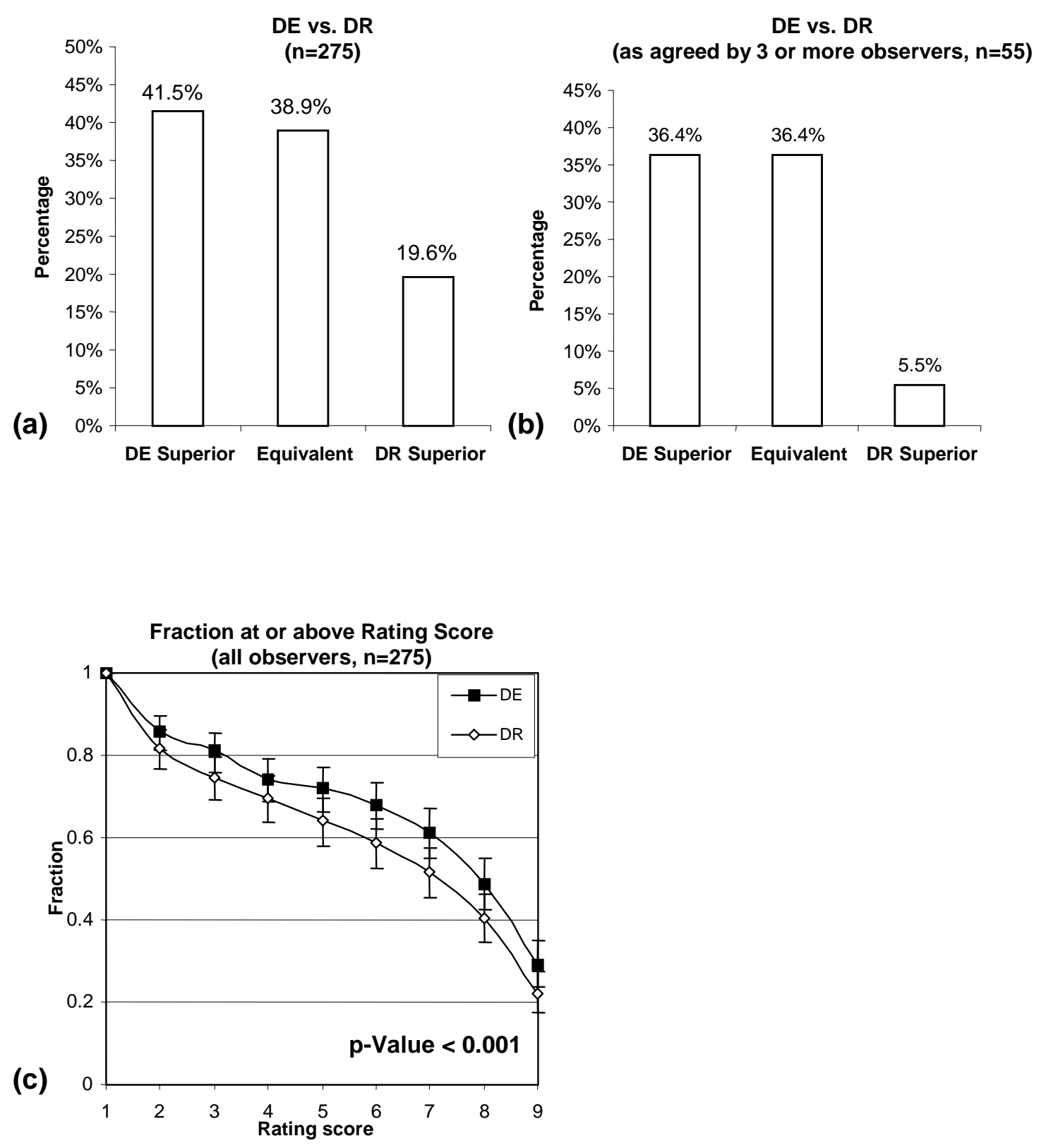

Figure 3.1 Diagnostic satisfaction in dual-energy (DE) and digital radiographic (DR) image readings (all cases pooled): (a) percentage of image pairs for which the DE image was rated superior, equal, or inferior to the DR image; (b) percentage of image pairs for which the DE image was rated superior, equal, or inferior to the DR image as agreed by three or more observers; (c) fraction of observer responses at or above a given rating score. The error bars represent $95 \%$ confidence intervals. 


\subsection{Stratification by Lesion Size}

The data were subsequently analyzed in terms of cases for which the lesion size was $\leq 3 \mathrm{~cm}$ and $>3 \mathrm{~cm}$ (36 and 19 cases, respectively, as shown in Table II). Results are shown in Fig. 3.2. A statistically significant improvement in diagnostic satisfaction was observed for DE imaging for nodules (lesion size $\leq 3 \mathrm{~cm}$, $\mathrm{p}$-value $<0.001$ ). The advantage of DE is more pronounced for nodules, as seen from the distinctly separated curves in Fig. 3.2(a) and the correspondingly smaller p-value.
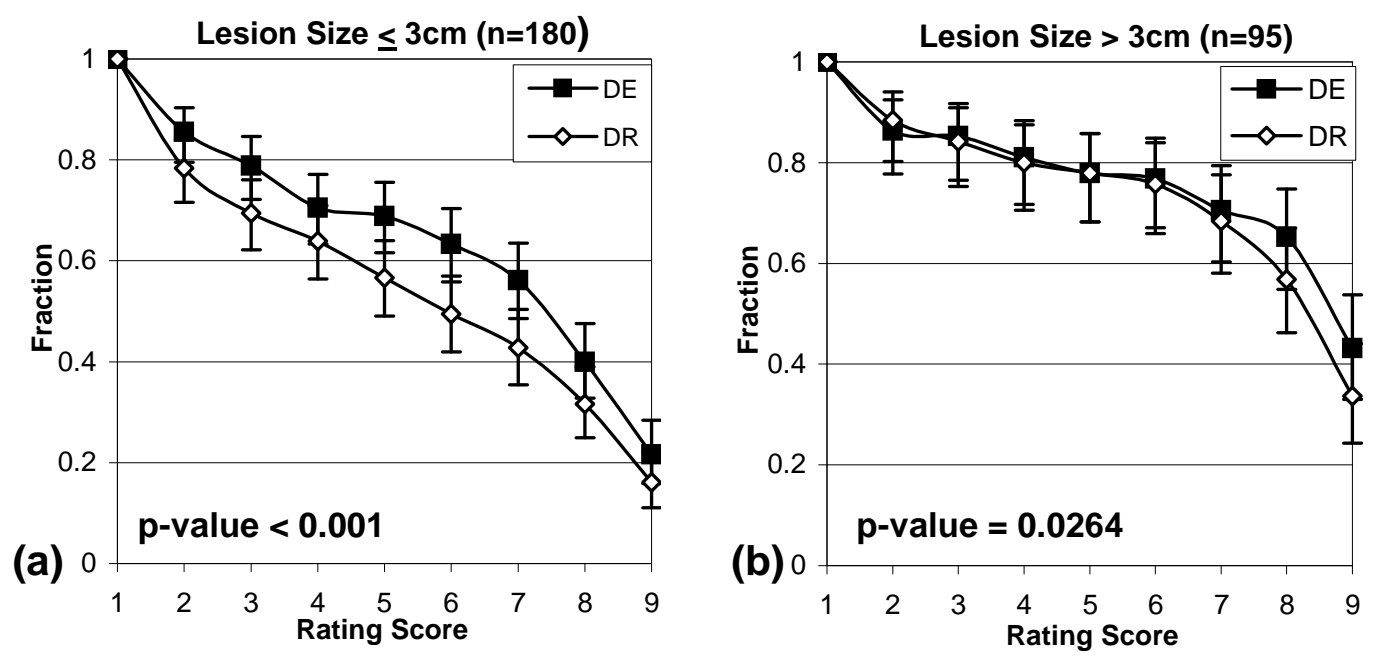

Figure 3.2 Diagnostic satisfaction in dual-energy (DE) and digital radiographic (DR) image readings stratified by lesion size. The curves show the fraction of responses at or above a given rating score for (a) lesion size $\leq 3 \mathrm{~cm}$ and (b) lesion size $>3 \mathrm{~cm}$. The error bars reflect $95 \%$ confidence intervals. 


\subsection{Stratification by Lesion Density}

Cases were stratified according to lesion density as solid $(\geq 20 \mathrm{HU})$ and non-solid $(<20 \mathrm{HU})$, with 46 and 9 cases, respectively (Table 3.2). A statistically significant improvement in diagnostic performance was observed for DE imaging of solid lesions (p-value $<0.001$ ). For non-solid lesions, DE and DR scores were not significantly different overall $(\mathrm{p}$-value $=0.0968)$ however, this finding may have been influenced by the low sample size of non-solid lesions in the pilot study. This finding will be more thoroughly investigated in future work using the entire patient cohort.
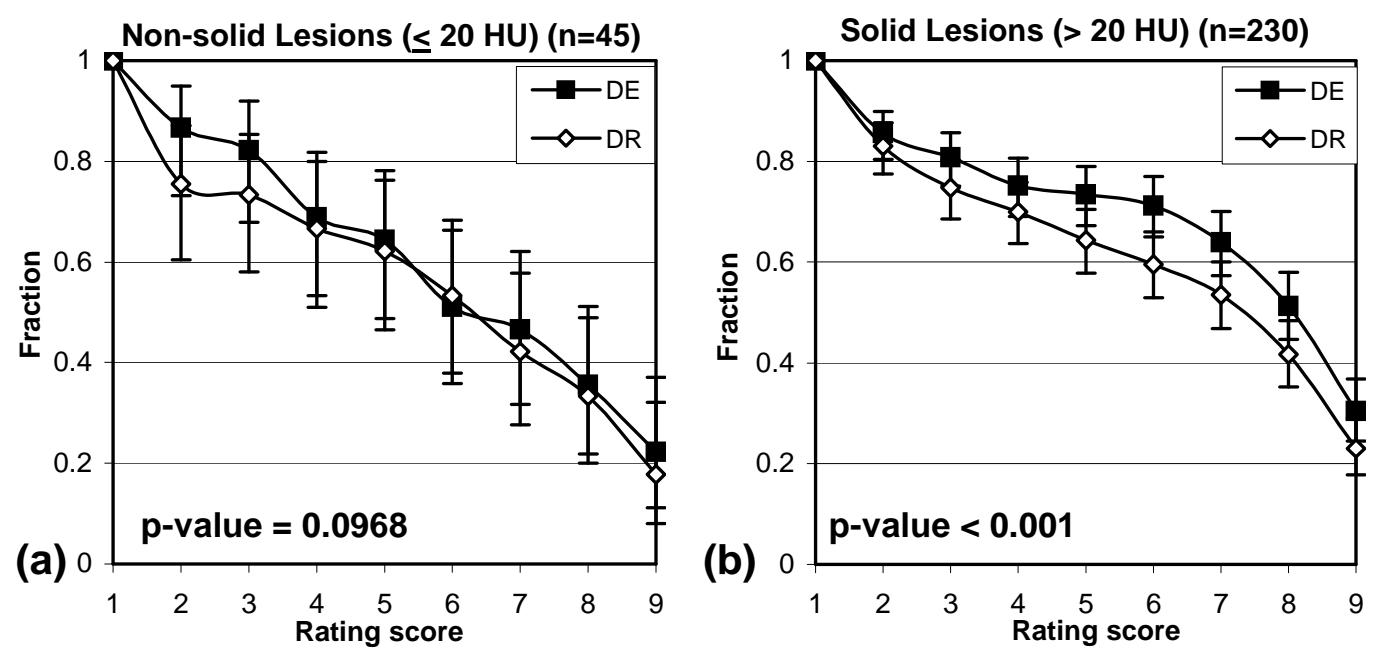

Figure 3.3 Diagnostic satisfaction in dual-energy (DE) and digital radiographic (DR) image readings stratified by lesion density. The curves show the fraction of responses at or above a given rating score for (a) nonsolid lesions ( $<20$ Hounsfield units [HU]) and (b) solid lesions (>20 HU). The error bars reflect $95 \%$ confidence intervals. 


\subsection{Stratification by Chest Thickness}

Results grouped according to PA chest thickness are shown in Fig. 3.4. A statistically significant boost was evident for DE imaging in the detection and characterization of lesions in both categories (viz., p-value $<0.0001$ for "average" and p-value $<0.002$ for "thick"). The curves indicate a fairly uniform improvement in diagnostic satisfaction for cases of "average" thickness (i.e., a uniform boost across all ratings), whereas for the "thick" cases, the curves appear to suggest a boost at the higher ratings (i.e., more conspicuous lesions). This observation suggests that the benefit of DE images may be somewhat less in "thick" than in "average" cases, presumably because images for the former are limited, at least in part, by quantum noise, contrast, $\mathrm{x}$-ray scatter, etc., rather than anatomical clutter.
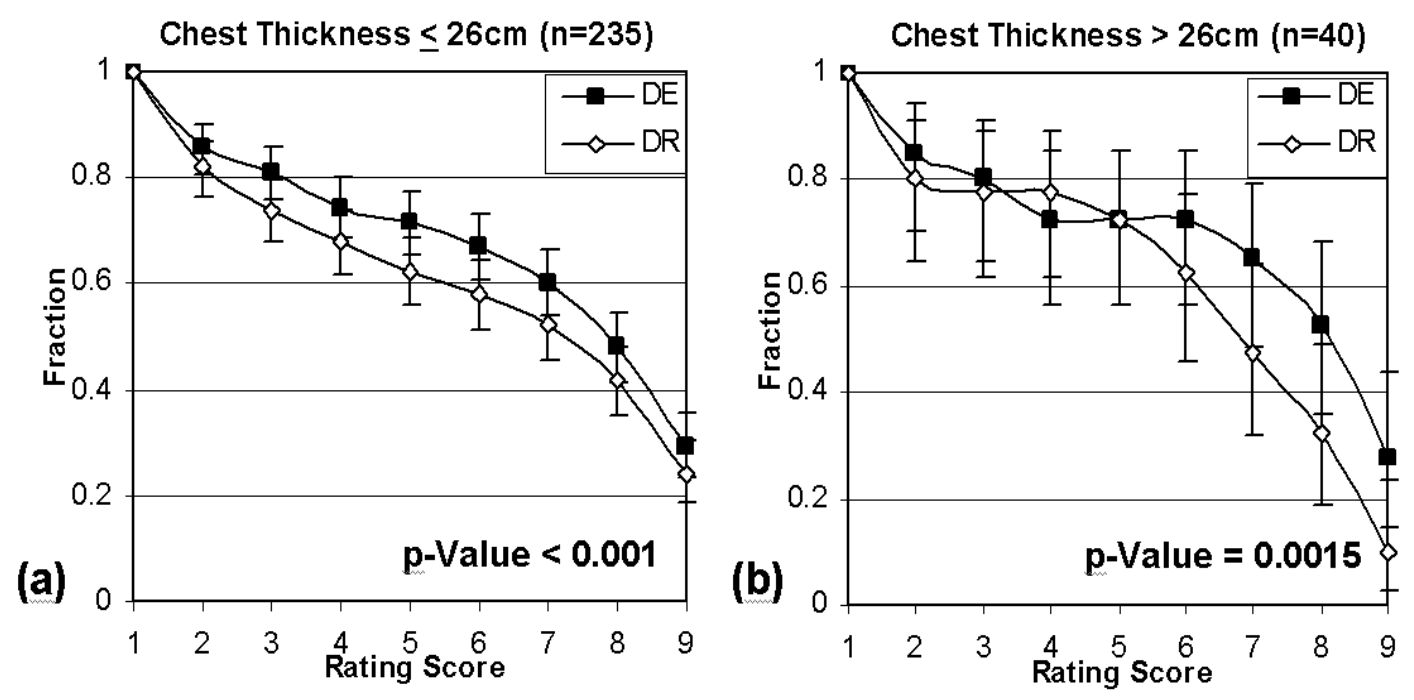

Figure 3.4 Diagnostic satisfaction in dual-energy (DE) and digital radiographic (DR) image readings stratified by chest thickness. The curves show the fraction of responses at or above a given rating score for (a) thickness $<26 \mathrm{~cm}$ and (b) thickness $>26 \mathrm{~cm}$. The error bars reflect $95 \%$ confidence intervals. 


\subsection{Stratification by Gender}

Cases were further grouped based on gender - a total of 31 male and 24 female patients. Results in Fig. 3.5 suggest a significant improvement in diagnostic performance for DE in each case (pvalue $<0.001$ for male and $\mathrm{p}$-value $=0.013$ for females). Such is likely consistent with the trend for improved performance overall for DE (Fig. 3.1). The smaller level of improvement suggested for the female sub-cohort (while still a statistically significant improvement) is possibly related to that observed for larger chest thickness (in this case, breast tissue), which correlated with a smaller improvement in diagnostic performance.
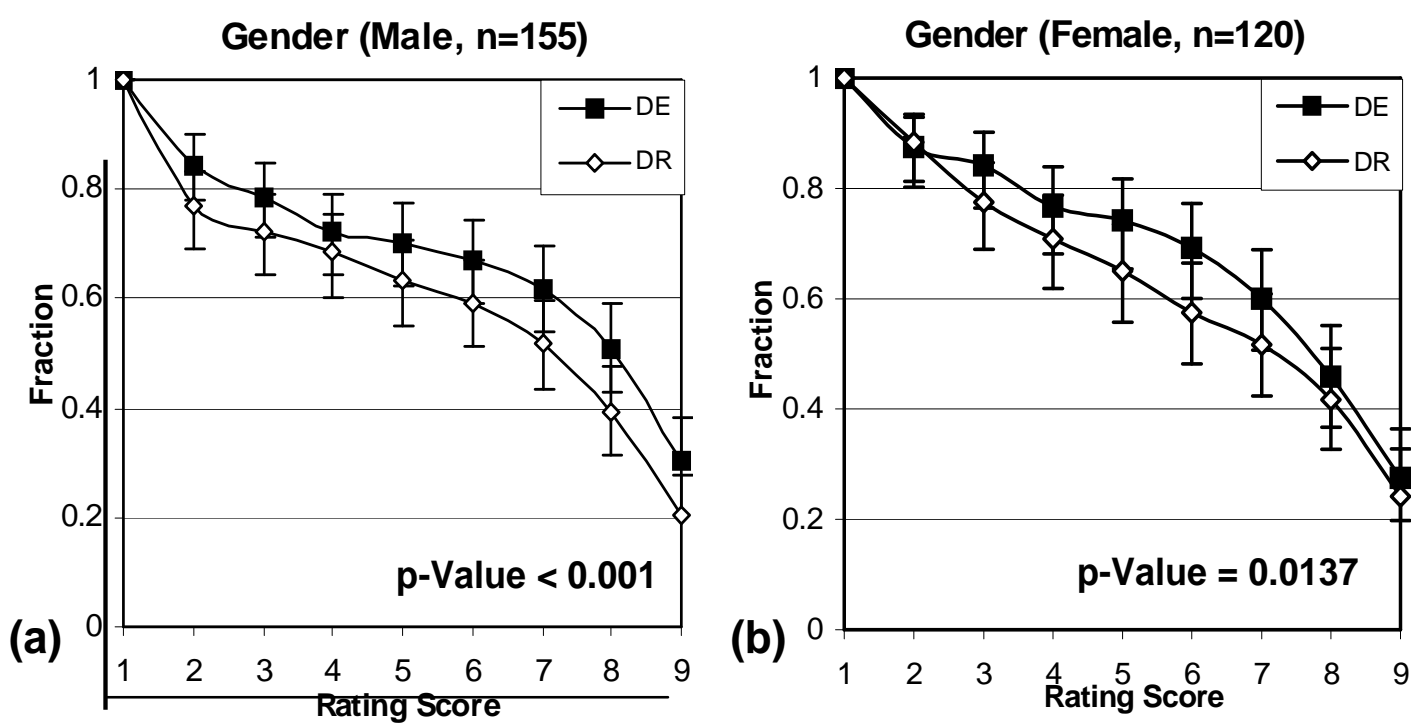

Figure 3.5 Diagnostic satisfaction in dual-energy (DE) and digital radiographic (DR) image readings stratified by patient gender. The curves show the fraction of responses at or above a given rating score for (a) men and (b) women. The error bars reflect $95 \%$ confidence intervals. 


\subsection{Stratification by Region}

Results grouped according to 7 regions of the chest (left and right apex, left and right middle, left and right lower, and mediastinal regions) are shown in Fig. 3.6. Although the number of nodules is relatively small in some of the sub-groups, therefore the findings should be viewed with some degree of caution, there is a trend for improved diagnostic performance for DE imaging in the left apex, right apex, left-middle, and left-lower regions. The results for the apical regions are consistent with the hypothesis that DE imaging improves diagnostic quality by removing anatomical noise - in this case, the clavicles and $1^{\text {st }}$ and $2^{\text {nd }}$ ribs, which exhibit complex anatomical clutter on frontal chest radiographs and can significantly diminish conspicuity. The significant improvement observed for $\mathrm{DE}$ imaging in the left-lower region is somewhat surprising, given that this region is challenged by a preponderance of soft-tissue structures (the heart) and is most susceptible to cardiac motion artifacts. Figures 3.7, 3.8 and 3.9 illustrate significant improvement of DE compared to DR in detection of a small solid nodule and nodules in right and left upper lobes. Images $\mathrm{a}, \mathrm{b}$ and $\mathrm{c}$ are illustrating examples of soft only, bone only and DR images. 

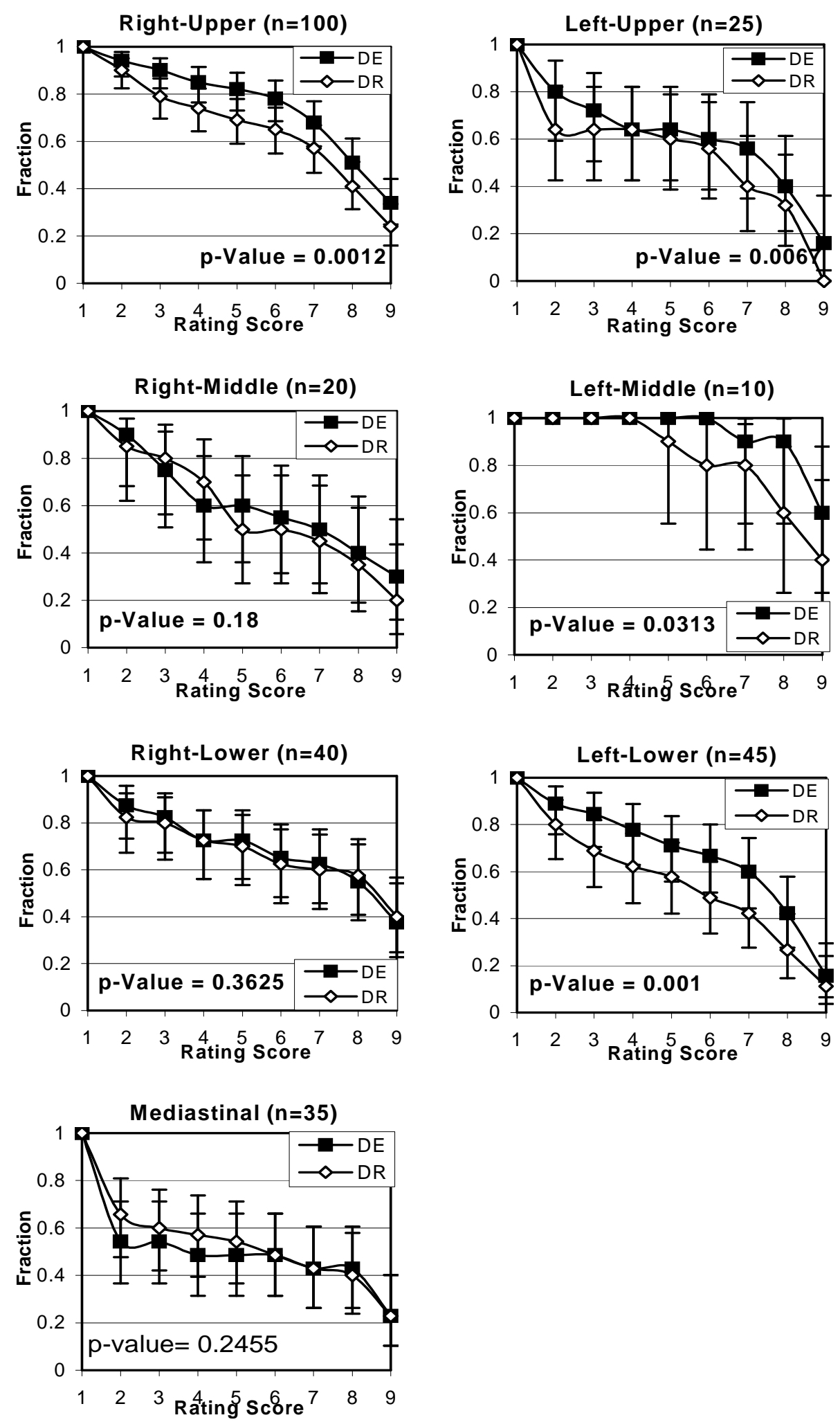
Figure 3.6 Diagnostic satisfaction in dual-energy (DE) and digital radiographic (DR) image readings stratified by lesion location. The curves show the fraction of responses at or above a given rating score for lesion located in (a) right upper, (b) left upper, (c) right middle, (d) left middle, (e) right lower, (f) left lower, and (g) mediastinal regions. The error bars reflect $95 \%$ confidence intervals. For lower satisfaction scores $(\leq 5)$ reflecting lower satisfaction, there is no clear pattern however for higher satisfaction scores $(>5)$, DE images demonstrate an advantage over DR.
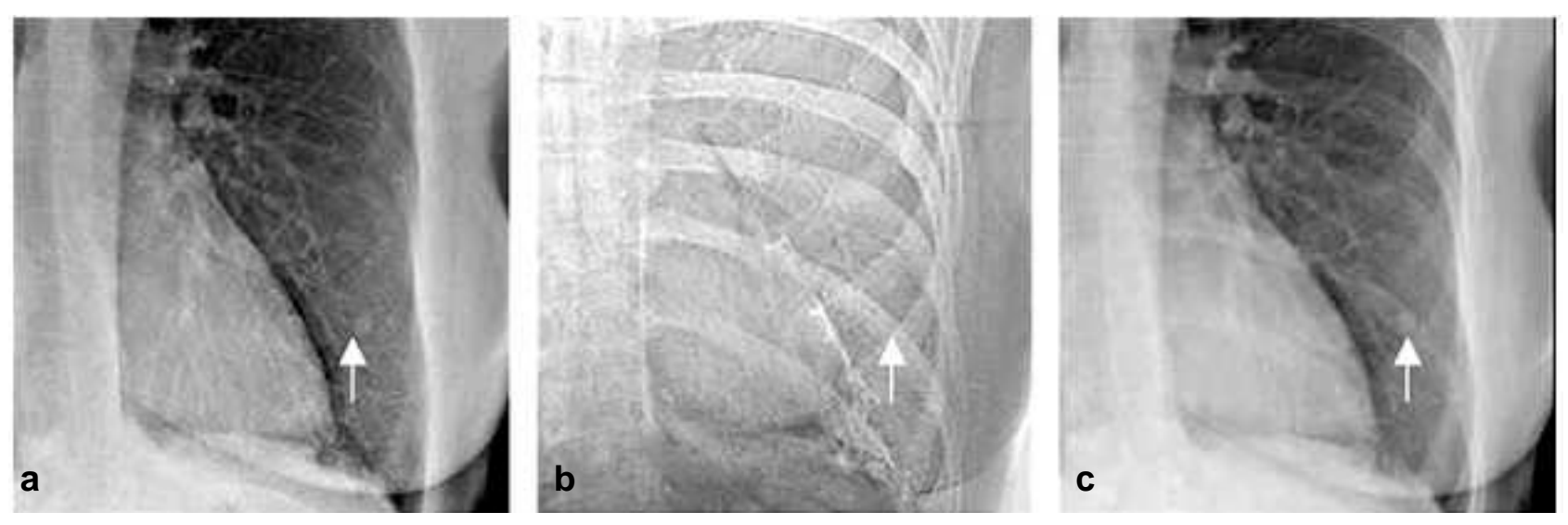

Figure 3.7 Illustration of (a) dual-energy soft tissue, (b) dual-energy bone, and (c) digital radiographic images for a case exhibiting a small, solid nodule $(0.8 \mathrm{~cm}, 58.4$ Hounsfield units, benign lymphoid tissue), marked by the arrows. The bone image exhibits some residual soft tissue attributable to incomplete cancellation as well as motion artifact, which is more obvious in this case because of the cardiac motion artifact. 

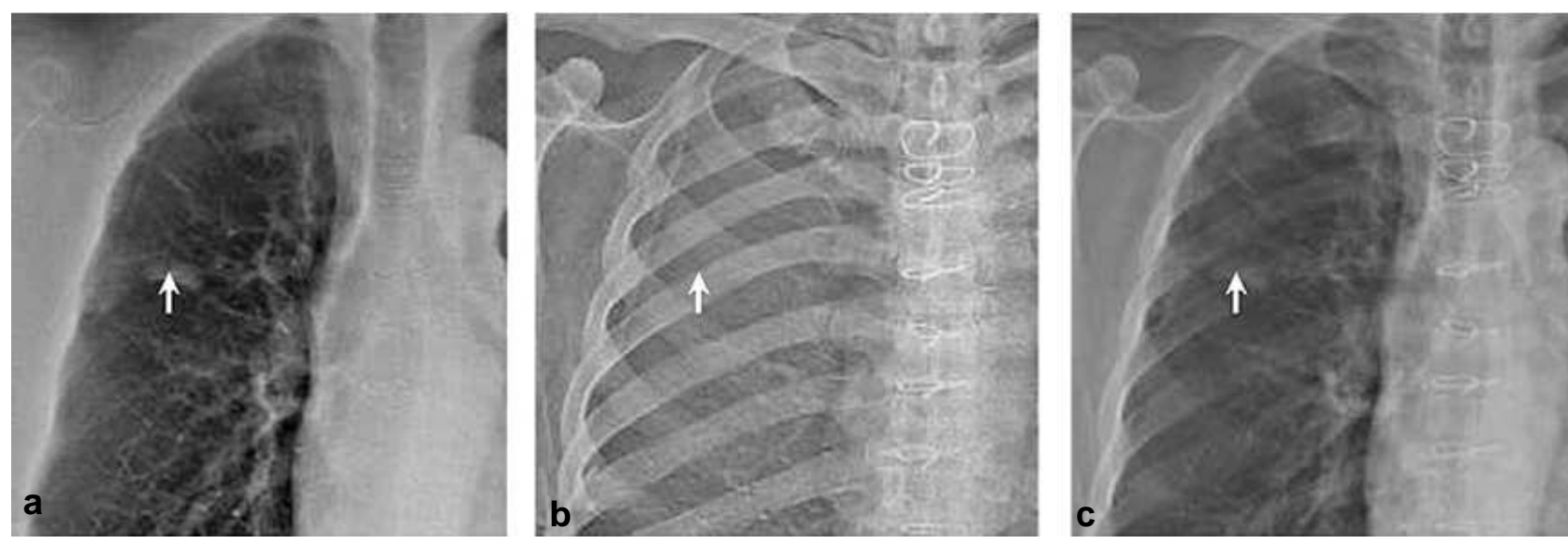

Figure 3.8 Illustration of (a) dual-energy soft tissue, (b) dual-energy bone, and (c) digital radiographic images for a case exhibiting a nodule $(2 \mathrm{~cm}, 67.5$ Hounsfield units, adenocarcinoma) in the right upper lobe, marked by the arrows.
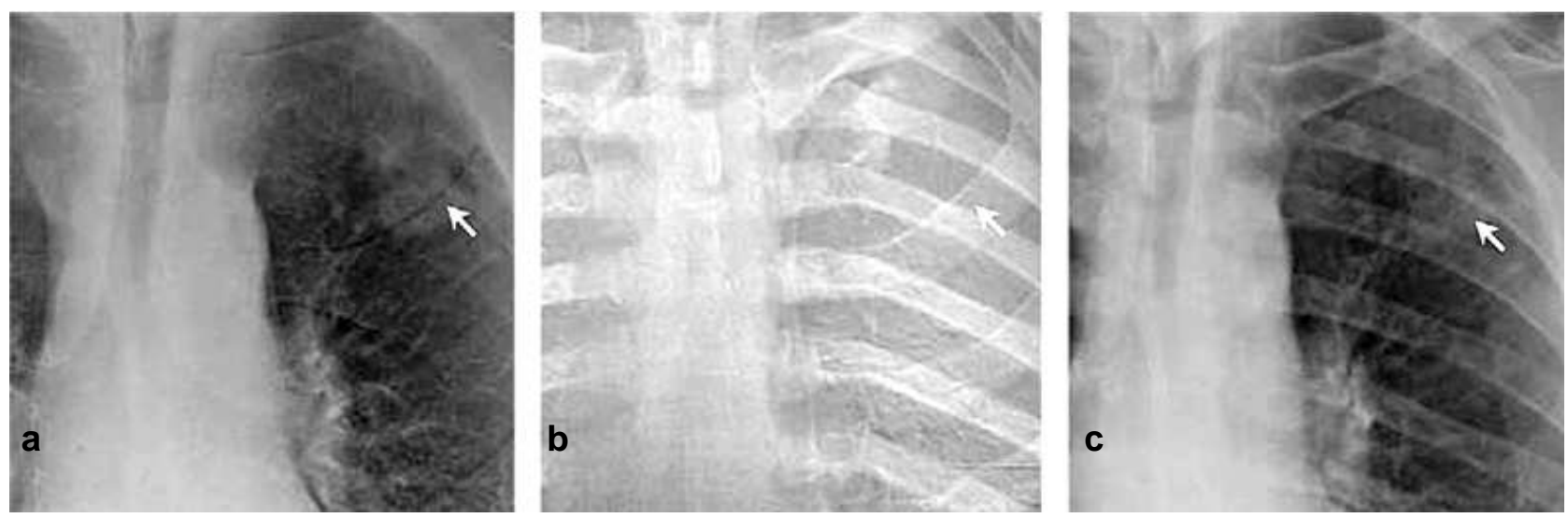

Figure 3.9 Illustration of (a) dual-energy soft tissue, (b) dual-energy bone, and (c) digital radiographic images for a case exhibiting a nodule $(2.8 \mathrm{~cm}, 35.1$ Hounsfield units, adenocarcinoma) in the left apex, marked by the arrows. 


\section{Chapter 4 ROC Test}

\section{Study Population}

As all 129 subjects were recruited from a population referred for biopsy of suspicious lung lesions (confirmed by CT prior to biopsy), all patients in the study cohort had at least one lung nodule. A pre-requisite of ROC analysis is to present a random series of images that contain the target and some that do not. Therefore, given the study cohort, the chest radiographs were divided to provide half-chest images, where each image was cropped near the vertical midline of the spine to present a complete image of just one lung. Although the presentation of half-chest images somewhat perturbs the usual presentation of chest images and therefore the usual radiologist reading pattern, observers reported no difficulty in assessing the cropped images.

Of the 129 cases, 29 exhibited bilateral disease; therefore, the 129 cases provided 258 half-chest images of which 158 contained nodules ("disease"), and 100 did not ("normal"). Of the 158 disease cases, 55 had multiple lesions, and 103 had only one lesion. The average size of the lesions (maximum diameter measured on CT) was $2.7 \mathrm{~cm}$, ranging from 0.7 to $14 \mathrm{~cm}$. The average density of lesions (measured in CT) was 11.5 HU, ranging from -769 HU to +180 HU.

Visualization software (termed "DEvice") was developed by the research team, to allow viewing of all three DE image components in a single, spatially co-registered view - i.e., soft-tissue, bone, and composite images viewed as a three-slice "stack". The software provided the ability to "scroll" between DE image components (using the mouse wheel) and to "see through" any given component to another (using a digital "looking glass" loupe invoked on the display) in addition to common image manipulation tools (e.g., window/level, magnification, pan, etc.). These software tools allowed fast, convenient visualization and comparison of anatomy presented 
among various image components. The composite image was the initial view (presenting a familiar radiographic scene with anatomical landmarks, etc.); via the mouse wheel (or the "looking glass" loupe), the reader could scroll to the soft-tissue and bone component images to more closely evaluate subtle image characteristics without the confounding influence of overlying anatomical noise from other tissue components. In this way, the display software better exposed the intrinsic potential advantage associated with multiple tissue components in DE imaging (without forcing the reader to pan between side-by-side images).

Five expert observers ( 3 staff radiologists and 2 radiology fellows, each a specialist in thoracic imaging) were independently presented with the images. The reading order was randomized, with one image presented at a time [either a DR image or a DE image (with all 3 components co registered in DEvice as described above)]. A diagnostic-quality monochrome LCD monitor (AM-QX21-A9300, National Display, San Jose, CA) calibrated to meet the DICOM grayscale standard was used. Studies were conducted in a dark-controlled radiology reading room ( 0.15 $\mathrm{Cd} / \mathrm{m}^{2}$ ambient light). Each observer was asked to rate each image in terms of the detection of abnormal lung lesions according to the 5-point scale in Table. 4.1. The scale was visible to observers on a second monitor. As the test was not a localization-specific ROC (LROC), observers were asked to score the lung image as a whole and did not record the exact location of the abnormalities. This imparts a potential limitation of the current study with respect to multiple nodules within one lung and the possibility of true-positive responses based on mistaken identification of a normal structure within an abnormal lung and not necessarily the abnormality itself. The possible error associated with this limitation is an overestimation of sensitivity, believed to be small (in part due to the conspicuity of actually-positive cases, as noted below). A training session involving 84 images (42 DE and $42 \mathrm{DR}$ ) was conducted prior to each observer study to familiarize the observers with the software. The training images were drawn from Group 
3 (non-cardiac-gated sub-group), which were the same as Group 1 in image acquisition and decomposition technique but did not overlap with cases used in the actual observer study. A viewing distance of $\sim 50 \mathrm{~cm}$ was encouraged but not enforced, and observers were free to adjust window/level, zoom, etc. as well as to scroll between DE image components or use the DE "looking glass." Tests required an average of $150 \mathrm{~min}$ to complete (18 sec per image), with one break permitted mid-way through the test.

\section{ROC Analysis}

\subsection{ROC Curves and Area under the Curve (AUC)}

Receiver Operating Characteristic (ROC) analysis which is frequently used to compare the diagnostic qualities of imaging systems (62) was performed using MedCalc (version 11.3, MedCalc Software bvba, Belgium) The resulting ROC curves plot the true-positive fraction (TPF, or sensitivity) versus the false-positive fraction (FPF, or 1-specificity), with each point along the curve representing TPF and FPF at a different decision threshold. For a five-point ROC rating scale as in Table 4.1, the corresponding four decision thresholds yield four measured control points on the ROC plot.(62)

\section{Receiver-Operating Characteristic Rating Scale}

1. Definitely normal ( or almost definitely normal)

2. Probably normal

3. Possibly abnormal

4. Probably abnormal

5. Definitely abnormal (or almost definitely abnormal)

Table 4.1 Five-point Rating Scale for Receiver-Operating Characteristic Studies 
The majority of ROC analysis used in radiological studies uses a discrete rating scale with five or six categories. The discrete five point rating scale has been compared to a continuous confidence scale by Rockette et al (63) and the measured area under the curve (AUC) was not significantly different between the two different methods, indicating that either could be used in image evaluation studies. Hadgiiski et al (65) examined the effects of the number of categories in the rating scale on the result of ROC. They concluded that the fitted curve and the performance indices do not vary significantly with different rating scales, only if the estimated operating points, obtained from the data, are relatively evenly distributed over the entire range of TPF and FPF. According to their results, ROC analysis of discrete confidence rating scales with few and unevenly distributed data points may cause unreliable estimation. The area under the curve is basically created by varying the cut point (decision making threshold) that is used to determine the values of the observed variables. If a test has a perfect discrimination capability, it would have a threshold value that would separate the entire abnormal population from the entire normal population (or vice versa). A graphical display would plot this kind of curve through $(0,1)(62)$. This would be the ideal point and as the observed curve conforms closer to this ideal form, it demonstrates improved discrimination between the normal and abnormal variables. If a test cannot discriminate at all it would follow a diagonal course within the grid. The area under the curve represents the probability that, if a randomly selected individual from the abnormal population and a randomly selected individual from the normal population are selected the observed values would be in correct order. Parametric assumptions are usually applied and the maximum likelihood method are used to estimate the area under the curve $(65,66)$ Figure 4.1 below shows the distribution of the observed values based on the 5 points rating scale, by all 5 observers and for two modalities, DE and DR. Since the observed values from our study did not follow a normal distribution, a non-parametric approach was chosen to estimate the AUC and to 
compare the areas under the correlated ROC curves with a non-parametric approach; trapezoidal rules apply to measure the area under the points comprising an empirical ROC curve. (67) The Mann-Whitney statistical test was used to compare the area under the two correlated curves.
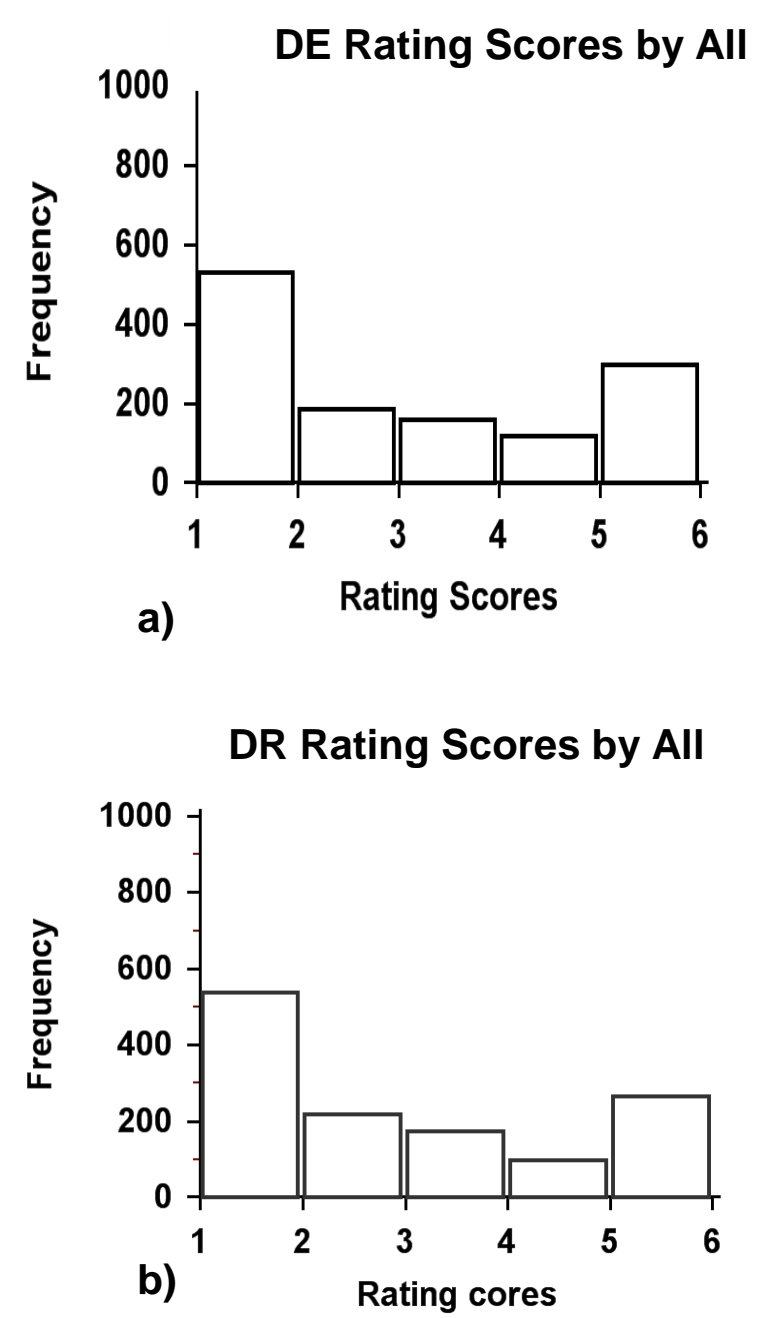

Figure 4.1 The distribution of rating scores among all observers for Dual energy (DE) and DR. As observed on the graphs, the distribution is non parametric and therefore non parametric ROC was applied. 
To measure the inter rater agreement, weighted kappa was used for rating scores of 5 observers and for each modality separately. The average weighted kappa for DE was calculated as $\mathrm{k}=0.51$ and for DR as $\mathrm{k}=0.52$. These numbers considered to fall under moderate agreement category. Performance was analyzed for all 258 cases pooled, and by post-hoc stratification of the data according to lesion size, lesion density, chest thickness, lesion location, and patient gender. Table 4.2 shows a summary of the strata. Results were analyzed for all strata by individual observer and for all observer responses pooled.

\begin{tabular}{|c|c|c|c|c|}
\hline Stratification & Description & DE & DR & How Determined \\
\hline Pooled & All cases & 258 & 258 & $\mathrm{~N} / \mathrm{A}$ \\
\hline \multirow[t]{3}{*}{ Lesion size } & Mass $(>3 \mathrm{~cm})$ & 44 & 44 & $\begin{array}{l}\text { Maximum linear dimension } \\
\text { measured in CT }\end{array}$ \\
\hline & Nodule $(\leq 3 \mathrm{~cm})$ & 114 & 114 & \\
\hline & Nodule $(\leq 1 \mathrm{~cm})$ & 18 & 18 & \\
\hline \multirow[t]{2}{*}{ Lesion density } & Solid ( $\geq 20 \mathrm{HU})$ & 128 & 128 & Mean HU measured in CT \\
\hline & Non-solid(<20 HU) & 30 & 30 & \\
\hline \multirow[t]{2}{*}{ Chest thickness } & $\operatorname{Large}(>26 \mathrm{~cm})$ & 68 & 68 & $\begin{array}{l}\text { AP distance (T9-xiphoid) measured } \\
\text { in axial CT }\end{array}$ \\
\hline & Average $(\leq 26 \mathrm{~cm})$ & 190 & 190 & \\
\hline \multirow[t]{7}{*}{ Lesion location } & Right-upper lobe & 48 & 48 & $\begin{array}{l}\text { Belonging to a given anatomical } \\
\text { region as visualized in } \mathrm{CT}\end{array}$ \\
\hline & Left-upper lobe & 29 & 29 & \\
\hline & Right-middle lobe & 8 & 8 & \\
\hline & Lingula & 4 & 4 & \\
\hline & Right-lower lobe & 26 & 26 & \\
\hline & Left-lower lobe & 30 & 30 & \\
\hline & Mediastinum & 13 & 13 & \\
\hline \multirow[t]{2}{*}{ Gender } & Male & 160 & 160 & Patient chart \\
\hline & Female & 98 & 98 & \\
\hline
\end{tabular}


Table 4.2 Summary of cases overall and within each post-hoc stratification.

\section{Results}

\subsection{Individual Observers}

The results of ROC tests for 5 individual observers (258 DE, 258 DR each) are summarized in Table 4.3, and the ROC curves for each observer are illustrated in Fig. 4.2, showing the fitted ROC as well as the coordinates (empiric ROC).

\begin{tabular}{lccc}
\hline \hline & AUC $_{\mathrm{DE}}$ & AUC $_{\mathrm{DR}}$ & P Value In AUC \\
\hline Observer 1 & 0.779 & 0.777 & 0.9345 \\
Observer 2 & 0.778 & 0.756 & 0.4279 \\
Observer 3 & 0.769 & 0.782 & 0.6519 \\
Observer 4 & 0.758 & 0.763 & 0.7633 \\
Observer 5 & 0.854 & 0.833 & 0.1842 \\
\hline \hline
\end{tabular}

Table 4.3 The area under the curve (AUC) for each observer is shown, found to be superior for dual energy (DE) imaging in three of five observers but not to a significant extent (two-tailed $P$ value based on $A \cup C)$.

The AUC for DE imaging was superior to DR for 3 out of 5 observers (two staff and one fellow) although the difference was not statistically significant. As described below, a similar result was found by pooling over all observers. 

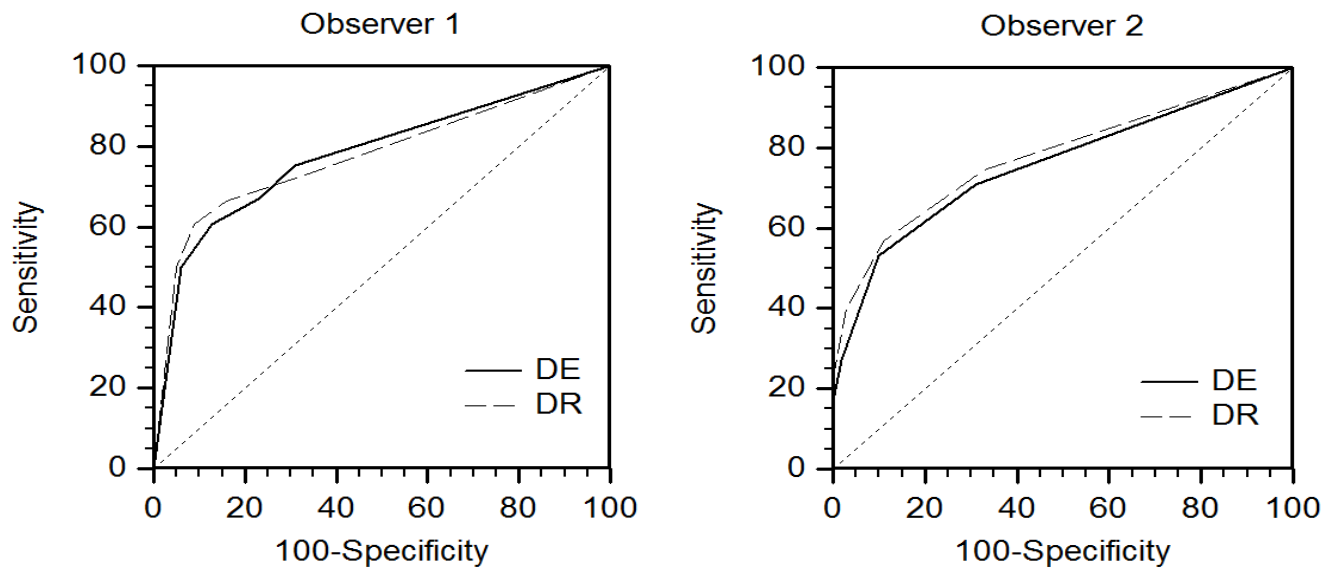

a) Observer 1

b) Observer 2
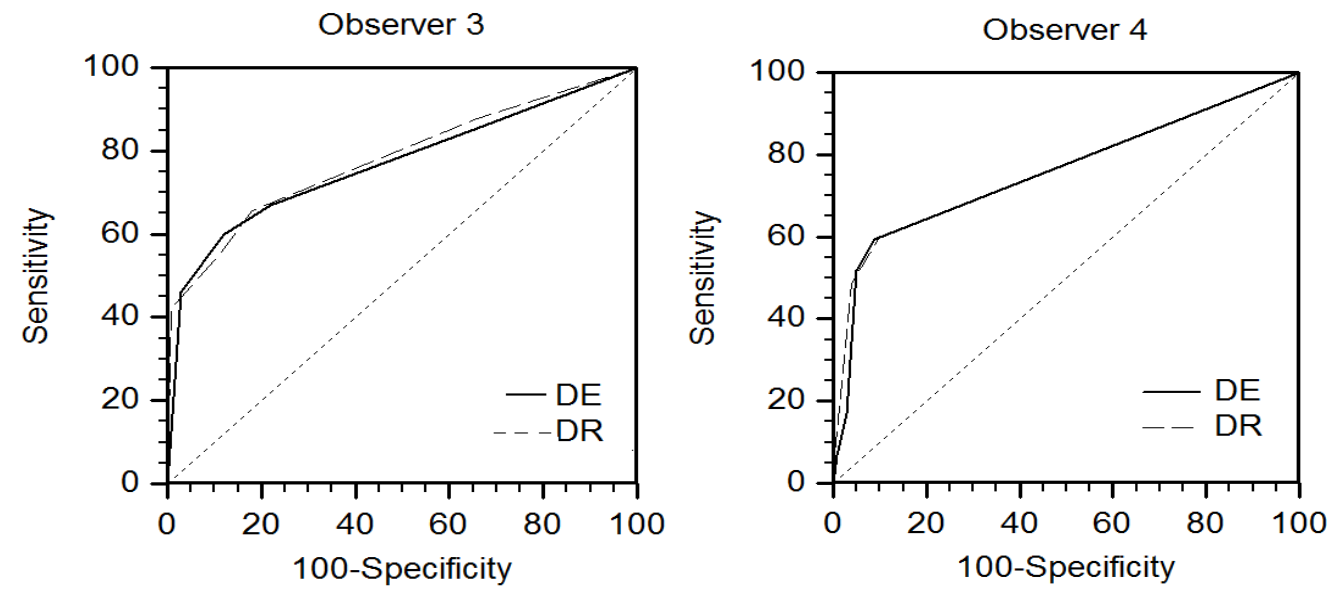

c) Observer 3

d) Observer 4

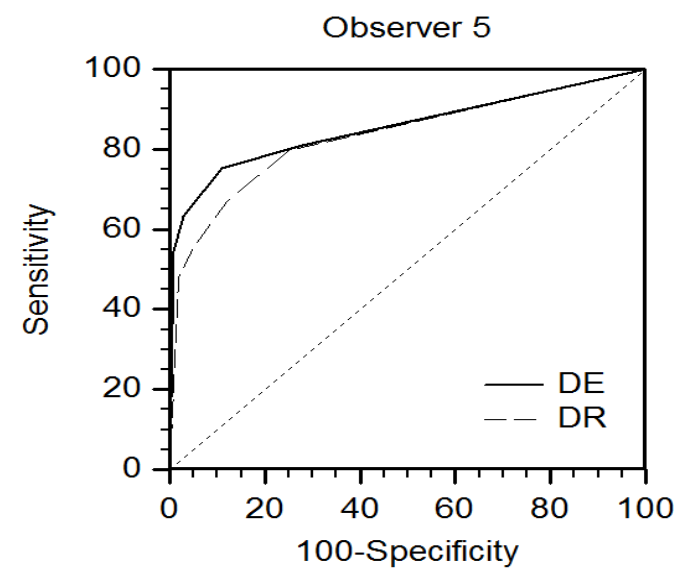

Figure 4.2 Receiver-operating characteristic (ROC) curves for five observers. Dual energy (DE) outperformed digital radiography (DR) for three of five readers $(a, b$, and e), although the difference in performance was not statistically significant $(P>.05)$.

\section{e) Observer 5}




\subsection{All Observers Pooled}

Results for the 1290 total ratings (5 radiologists x 258 cases) for each of the two modalities (DE and DR) are summarized in Table 4.4. The statistical significance in observed differences was evaluated in terms of the entire AUC (total area under the curve). Overall, the AUC for DE imaging was higher than DR in the following cases: all cases pooled; small chest thickness; female; nodule diameter $\leq 3 \mathrm{~cm}$; solid nodules; and nodules located in the right-upper lobe (RUL) and right-lower lobe (RLL). However, the difference was statistically significant only for cases of nodule diameter $\leq 1 \mathrm{~cm}$ (p-value=0.02), and RUL (p-value $=0.002)$. A statistically significant difference was also seen in the LLL but here DR outperformed DE (p-value=0.05).

\begin{tabular}{|c|c|c|c|}
\hline Stratification & Description & $A U C_{D E}$ & $\mathrm{AUC}_{\mathrm{DR}}$ \\
\hline Pooled & All cases & 0.781 & 0.776 \\
\hline \multirow[t]{3}{*}{ Lesion size } & Mass $(>3 \mathrm{~cm})$ & 0.797 & 0.822 \\
\hline & Nodule $(\leq 3 \mathrm{~cm})$ & 0.775 & 0.758 \\
\hline & Nodule $(\leq 1 \mathrm{~cm})$ & 0.758 & 0.689 \\
\hline \multirow[t]{2}{*}{ Lesion density } & Solid ( $\geq 20 \mathrm{HU})$ & 0.805 & 0.798 \\
\hline & Non-solid (<20 HU) & 0.678 & 0.682 \\
\hline \multirow[t]{2}{*}{ Chest thickness } & Large $(>26 \mathrm{~cm})$ & 0.741 & 0.758 \\
\hline & Average $(\leq 26 \mathrm{~cm})$ & 0.798 & 0.784 \\
\hline \multirow[t]{7}{*}{ Lesion location } & Right upper lobe & 0.804 & 0.759 \\
\hline & Left upper lobe & 0.801 & 0.808 \\
\hline & Right middle lobe & 0.808 & 0.839 \\
\hline & Lingula & 0.898 & 0.933 \\
\hline & Right lower lobe & 0.770 & 0.738 \\
\hline & Left lower lobe & 0.766 & 0.806 \\
\hline & Mediastinum & 0.578 & 0.609 \\
\hline \multirow[t]{2}{*}{ Gender } & Male & 0.773 & 0.775 \\
\hline & Female & 0.798 & 0.782 \\
\hline
\end{tabular}


Table 4.4 The total number of readings for each modality was 1290 (equal to 258 cases - 5 radiologists), with 790 actually positive and 500 actually negative cases. Results were analyzed in terms of the AUC for dual energy (DE) imaging and digital radiography (DR) (area under the curve AUC DE and AUC DR, respectively).Statistical significance in the observed differences were analyzed in terms of the P value associated with the entire AUC. Statistically significant results are highlighted in bold text.

The ROC curves for the two strata with statistically significant difference between the two modalities are illustrated in Fig. 4.3. For small nodules (diameter $\leq 1 \mathrm{~cm}$, Fig. 4.3a), DE imaging exhibited improved AUC (p-value=0.03). This observation suggests a clinical advantage in discriminating small, benign lesions with DE. The improvement in AUC for DE demonstration of lesions in the RUL (Fig. 4.3b) is consistent with the notion that DE imaging improves delectability in regions dominated by anatomical clutter; in this case the ribs and clavicles. The lack of a similar finding in the LUL may be due to the smaller number of cases in that stratum. Figures 4.4 and 4.5 show example images for which DE imaging clearly outperformed DR, cases of a small nodule (diameter $\leq 1 \mathrm{~cm}$ ) and a nodule in the RUL, respectively.

\begin{tabular}{|c|c|c|c|}
\hline & AUC & SE & $95 \% \mathrm{Cl}$ \\
\hline DE & 0.758 & 0.0293 & 0.700 to 0.815 \\
\hline DR & 0.689 & 0.0310 & 0.628 to 0.750 \\
\hline \multicolumn{3}{|c|}{ Difference between areas } & 0.0684 \\
\hline \multicolumn{3}{|c|}{ Standard Error } & 0.0295 \\
\hline \multicolumn{3}{|c|}{ 95\% Confidence Interval } & 0.0105 to 0.126 \\
\hline \multicolumn{3}{|c|}{ z statistic } & 2.317 \\
\hline \multicolumn{3}{|c|}{ Significance level } & $P=0.0205$ \\
\hline
\end{tabular}

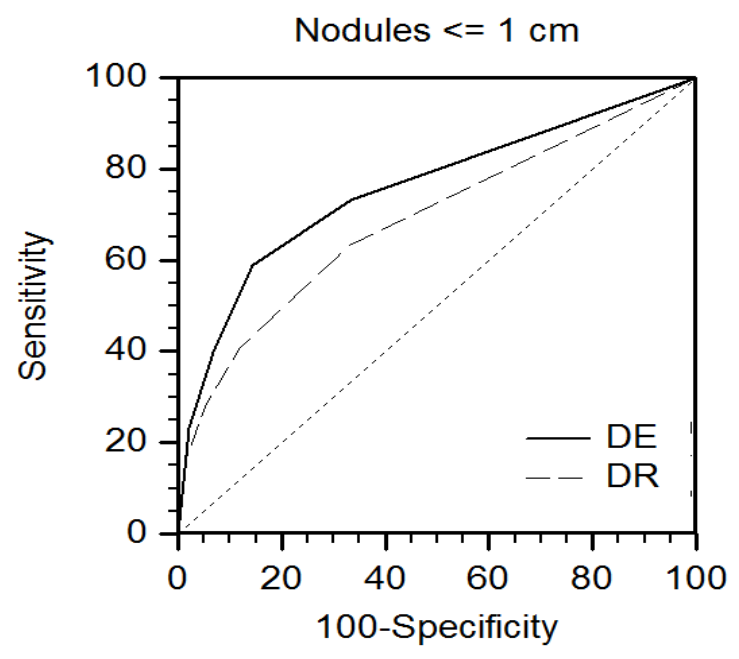

a) 


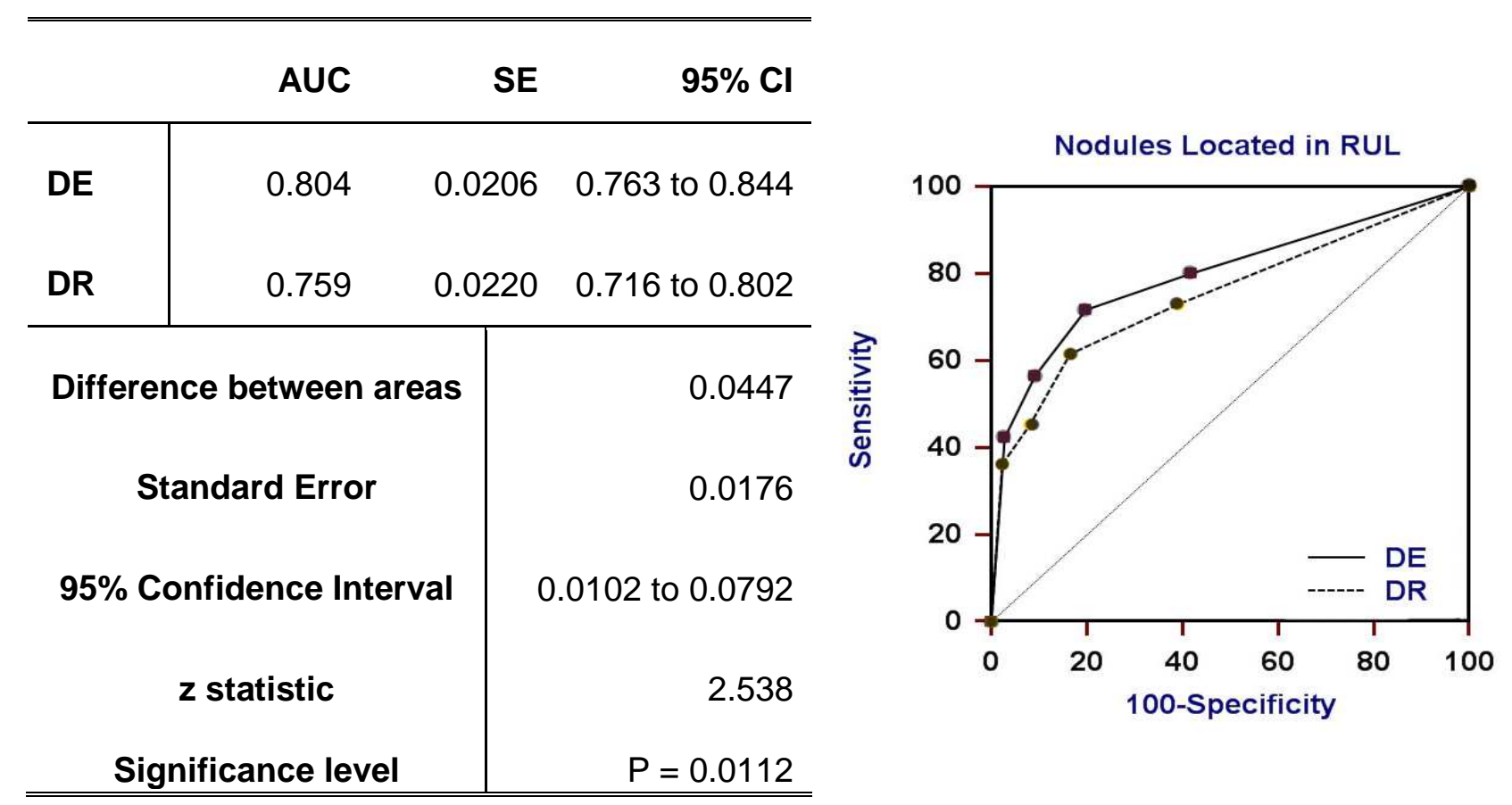

b)

Figure 4.3 a, b. Receiver-operating characteristic (ROC) curves for five nodules smaller than 1 mm (a) and nodules located in the Right Upper Lobe. (b) DE outperformed DR in these two categories $(P<.05)$
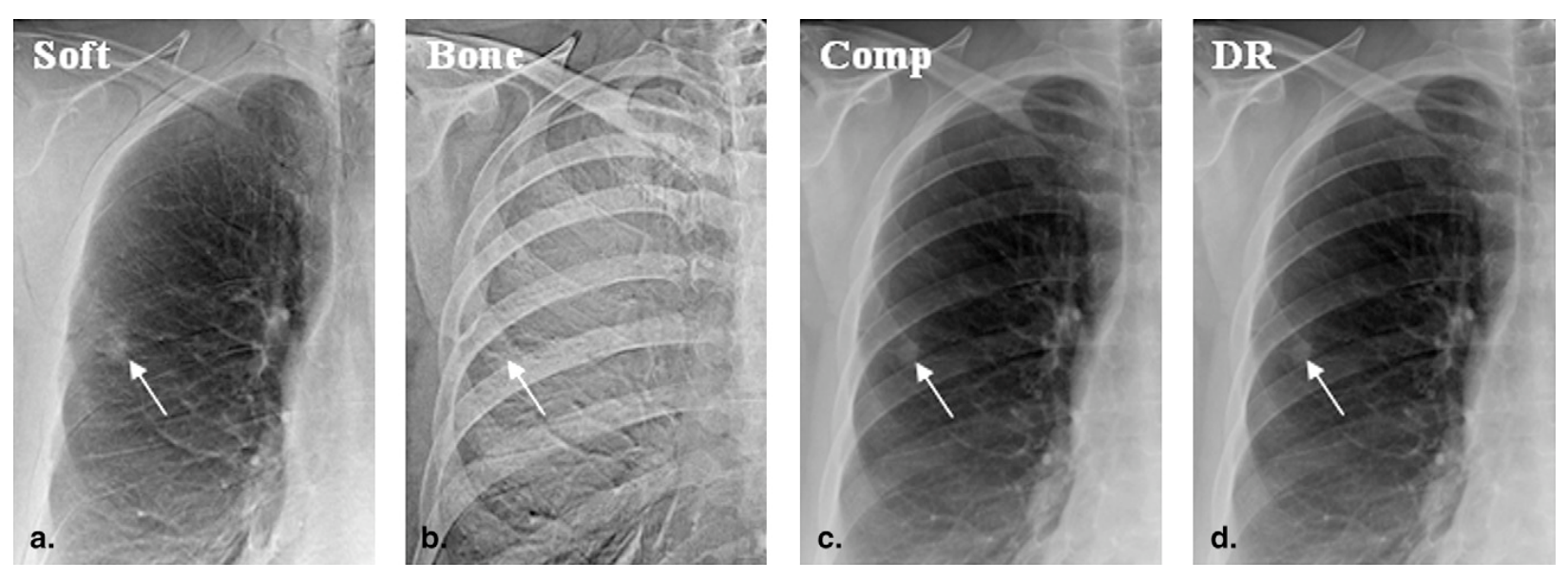

Figure 4.4 Example of dual energy (DE) and digital radiography (DR) images for a small nodule. The location of a $0.9 \mathrm{~cm}$ nodule in the right upper lobe with density of $28.5 \mathrm{HU}$ is marked by an arrow. 

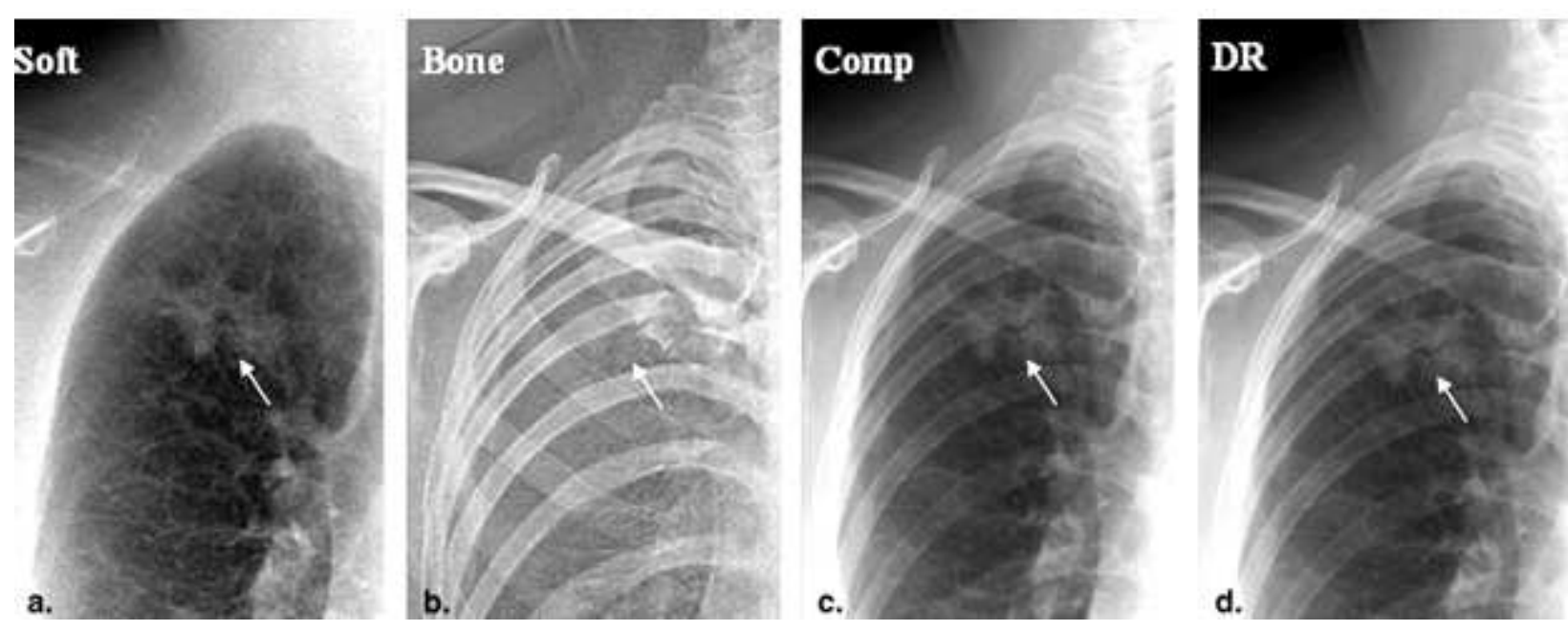

Figure 4.5 Example of dual energy (DE) and digital radiography (DR) images for a nodule located in right upper lobe. The location of a 3-cm nodule with density of $42.9 \mathrm{HU}$ is marked by an arrow.

\subsection{Diagnostic Accuracy and Clinical Significance}

Table 4.5 shows the overall accuracy of DE and DR imaging in discriminating abnormal and normal cases. For the purposes of this study, accuracy was defined as the capability of the reader to correctly identify a case as true-positive or true-negative (i.e., a rating of 1 or 2 in normal cases or 3-5 in abnormal cases). The term "accuracy" as defined here is equivalent to the fraction of correctly identified cases as commonly defined [(TP+TN) / $(\mathrm{TP}+\mathrm{TN}+\mathrm{FP}+\mathrm{FN})]$, a reasonable figure of merit for tests in which the number of normal and abnormal (disease) cases are approximately the same. For disease cases, $\sim 0.31$ (49/158 readings) were missed in DR but were properly identified in DE. Conversely, 0.26 (41/158) of disease cases were missed in DE but were properly identified in DR [DE and DR agreed (i.e., both $\geq 3$ or both $\leq 2$ ) in the remainder of cases.] These data suggest slightly improved sensitivity for DE imaging to an extent that could avoid false negative findings in $\sim 5 \%(8 / 158)$ of cases. For normal studies, 0.24 (24/100) were false positive cases in DR but were properly identified in DE. Conversely, 0.33 (33/100) were false positive cases in DE but were properly identified in DR. These data suggest a reduction in 
specificity associated with the increased sensitivity of DE imaging to an extent that would cause false-positive readings in $9 \%(9 / 100)$ cases. While such tentative results would benefit from a dedicated multi-center trial (in which the importance of the bone image in detecting subtle calcification might become more evident), they suggest the extent to which DE imaging could indicate a clinically distinct diagnostic path for patients, suggestive of a clinically significant difference in up to $\sim 10 \%$ of patients, with $\sim 5 \%$ of patients benefiting from early nodule detection.

Table 4.5 also compares the overall definitiveness of DE and DR imaging in identifying disease and normal cases. The definitiveness is defined as the highest level of reader confidence in identifying or rejecting an abnormality (i.e., a rating of 1 or 5). The number of cases "definitively" identified in one modality was analyzed (i.e., a rating of 1 or 5 for $\mathrm{TP}$ or TN, respectively) relative to a less definitive score for the other modality (i.e., a rating $\geq 2$ or $\leq 4$ for disease or normal cases, respectively). For disease cases, $\sim 0.29$ (46/158 readings) were more definitively identified in DE, compared to 0.18 (29/158) for DR. These data suggest an improvement in the definitiveness of positive diagnosis for DE compared to DR, where DE imaging gave more definitive scores in $\sim 11 \%$ of cases $(17 / 158)$ that were either missed or were less certainly scored using DR. Normal cases, 0.46 (46/100) were more definitively rejected (ROC score $=1)$ by DE imaging than in DR, compared to $0.44(44 / 100)$ more definitively identified in DR. These data suggest a slight improvement in definitive specificity for DE to an extent that avoided false positive readings in $2 \%$ of cases $(2 / 100)$. Improved definitiveness of diagnosis (in both normal and disease cases) may be particularly important in clinics without access to $\mathrm{CT}$, and it may present a cost-effectiveness advantage by reducing the transfer of suspicious cases to better equipped centers. 


\begin{tabular}{|c|c|c|c|c|c|}
\hline Accurate & & $\begin{array}{l}\mathrm{DE} \geq 3 \\
\text { (Correct) AND } \\
\mathrm{DR} \leq \\
2 \text { (Wrong) }\end{array}$ & $\begin{array}{l}\mathrm{DE} \leq 2 \\
\text { (Wrong) AND } \\
\mathrm{DR} \geq 3 \text { (Correct) }\end{array}$ & $\begin{array}{l}\mathrm{DE} \leq 2 \\
\text { (Correct) AND } \\
\mathrm{DR} \geq 3 \\
\text { (Wrong) }\end{array}$ & $\begin{array}{l}\text { DE } \geq 3 \text { (Wrong) } \\
\text { AND } \\
D R \leq 2 \\
\text { (Correct) }\end{array}$ \\
\hline & $\begin{array}{l}\text { Fraction } \\
\text { (Number } \\
\text { of } \\
\text { readings) }\end{array}$ & $0.31(49 / 158)$ & $0.26(41 / 158)$ & $0.24(24 / 100)$ & $0.33(33 / 100)$ \\
\hline \multirow[t]{2}{*}{ Definitive } & & $\begin{array}{l}\mathrm{DE}=5 \text { AND } \\
\mathrm{DR} \leq 4\end{array}$ & $\begin{array}{l}\mathrm{DE} \leq 4 \mathrm{AND} \\
\mathrm{DR}=5\end{array}$ & $\begin{array}{l}\mathrm{DE}=1 \mathrm{AND} \\
\mathrm{DR} \geq 2\end{array}$ & $\begin{array}{l}\mathrm{DE}>2 \text { AND } \\
\mathrm{DR}=1\end{array}$ \\
\hline & $\begin{array}{l}\text { Fraction } \\
\text { (Number } \\
\text { of } \\
\text { readings) }\end{array}$ & $0.29(46 / 158)$ & $0.18(29 / 158)$ & $0.46(46 / 100)$ & $0.44(44 / 100)$ \\
\hline
\end{tabular}

Table 4.5 Accuracy, Definitiveness, and Clinical Significance, Dual energy (DE) imaging was more accurate than digital radiography (DR) in identifying actually positive cases but less accurate in ruling out actually negative cases. DE imaging was more definitive in properly identifying both actually positive and actually negative cases. 


\section{Chapter 5 \\ Conclusion and Future Work}

DR imaging of the chest represents a cost-effective, widely available, low-dose modality used for a broad spectrum of applications, ranging from bedside exams to the initial examination and diagnosis of lung disease. Still, it is known to suffer in sensitivity for the detection of subtle lesions, limited primarily by a lack of conspicuity caused by superimposition of anatomic structures. DE imaging, which reduces anatomic clutter by selectively removing material specific components from the image, showed a significant improvement in satisfaction ratings associated with the detection and characterization of pulmonary nodules. DE imaging by selective decomposition and visualization of soft tissue and bone images reduces anatomical noise and demonstrates an increased sensitivity for the detection of lung nodules especially in the areas that are challenging for DR, such as the lung apex (68). This study evaluated the performance of DE imaging using a preference test and an ROC study involving 5 observers. The preference test was initially evaluated in 55 patients drawn from an ongoing trial; it provided initial investigation of diagnostic performance and supported the hypothesis that DE imaging boosts lesion conspicuity. The differences in satisfaction between DE and DR imaging are shown in Figures 3.1 to 3.6, with qualitative differences illustrated in the patient images in Figures 3.7 to 3.9. For example, the difference observed for small, solid nodules ( $<3 \mathrm{~cm}$ diameter, $20 \mathrm{HU}$ density) in Figures 3.2 and 3.3 is illustrated qualitatively in Figure 3.7. The improved visualization of small, solid nodules by virtue of rib cancellation is clear. Figures 3.8 and 3.9 similarly illustrate improved nodule visibility in the lung apex, in which the clavicles, first and second ribs present confounding clutter that is significantly reduced in the DE soft tissue image. That the difference in performance was greatest for small, apical solid nodules is particularly valuable, because these characteristics represent precisely the most challenging (i.e., small), important (i.e., solid, more 
likely malignant), and frequent (i.e., location in lung apex) cases and the areas most in need of improvement in chest imaging.

The initial results were encouraging, although the study was not without its limitations. First, the number of cases was low, particularly for certain stratifications and sub group analysis (e.g., anatomic region), because these early data constitute an initial study from an ongoing trial. Furthermore, the stratifications within the data were post hoc, and although the overall study was prospectively designed to investigate the difference in performance between DE and DR imaging, the data pertaining to individual strata (i.e., nodule size, nodule density, chest thickness, gender, and nodule location) should be considered "hypothesis generating' in the sense of retrospective analysis. Furthermore, the strata were distinct and independent (e.g., grouping small or solid or apical nodules, but not small, solid, and apical nodules), and joint grouping was not examined. Second, the satisfaction test on the basis of a nine-point scale has not been validated in a wider range. Although the intra observer agreement was testes for all observers at the beginning of the study and the repeat readings were found to be highly reproducible, the inter observer variability and validity of the test was not quantified. Two previous studies have found the same approach to generate consistent results between the readers but a thorough evaluation of the overall strength of the psychometric analysis of this method has never been performed. The Statistical significance was evaluated in terms of P values obtained from Wilcoxon's signed-rank tests. A limitation associated with clustering effects within ratings for the same patient across observers is possible. A third limitation of the study involves the DE image processing and decomposition. Although the DR images were post processed according to techniques established for optimal clinical DR imaging, the DE image post processing was fairly simple. The DE images used in the observer study used the simple image processing and decomposition techniques specifically, single-point offset and gain corrections, non-optimized registration of 
low- and high-energy projections, and simple log weighted subtraction according to a scalar tissue cancellation parameter.

Overall, DE imaging had a superior diagnostic performance in the satisfaction test than DR imaging at equivalent radiation dose. This was mainly attributed to the soft tissue images that present subtle lesions more conspicuously by virtue of reduced anatomic clutter. Although the observer response (rating) pertained to the combined DE image set (i.e., the soft tissue and bone images considered together, rather than each rated individually), it was clear that the soft tissue image was the more important in nodule detection, while the bone image presented complementary information regarding characterization (e.g., calcification). For this patient cohort in particular (drawn from a clinical patient population referred for a lung nodule biopsy), there were few cases exhibiting calcified nodules. Therefore, the bone images were likely used less than would be the case in a general screening population (in which the frequency of calcified nodules would presumably be greater). Furthermore, the bone image could provide diagnostic value regarding bony pathology, for example in differentiation of rib metastasis from rib fracture and improved visualization of fine bony detail to exclude cortical invasion.

The ROC study evaluated the performance of DE imaging involving 5 observers and 129 cases with half-chest images, resulting in 258 images. DR images were compared to DE images consisting of three components: a soft-tissue image, a bone image, and a composite image comparable to DR. The techniques were compared by measuring the total area under the curve (AUC) in an ROC analysis. Overall, DE outperformed DR in detection of nodules smaller than 1 $\mathrm{cm}$, and nodules in the RUL. Superior diagnostic performance for DE imaging in the RUL was likely due to the removal of anatomical noise related to the clavicle, first, and second ribs in this region which cause significant limitations in detection of parenchymal abnormality by DR. This 
problem is reduced through tissue decomposition techniques (i.e., bone cancellation) in DE imaging. It is therefore somewhat surprising that similar results were not realized for lesions in the LUL, which could reflect the smaller number of cases with LUL lesions in this study cohort.

The results were promising, but the study was not without limitations. First, the study subjects were recruited from a population suspicious for lung cancer and referred for CT-guided percutaneous lung biopsy. Most of these cases had lung nodules that were initially detected on CR or DR chest radiographs and therefore had fairly conspicuous nodules; this observation might have limited the potential advantage of DE imaging. However, DE imaging demonstrated a relatively high sensitivity and specificity for small $(<1 \mathrm{~cm})$ nodules. This technique may be useful in evaluating high-risk populations for lung malignancy - for example, cigarette smokers, who exhibit a high incidence of small nodules on low dose CT, which is known to be sensitive but non-specific. The vast majority of nodules detected on low-dose CT are benign, but detection of these nodules generates significant expense, the studies are time-intensive to read, and are performed at a relatively high radiation dose. Dual-energy imaging has the potential to overcome many of these deficiencies and also demonstrates superior performance to DR imaging for RUL nodules, an area where early lung cancers are commonly detected late due to obscuration by the overlying rib or clavicle.

Second, the observers as chest radiologists were all aware of the modality they were evaluating during the study (due to specific features of each modality) and the use of half-chest images, although necessary for the purposes of this study, is a distortion of the usual clinical reading pattern used by thoracic radiologists. These aspects of the study could have biased the results, presumably to an equivalent degree for both DE and DR. 
The third limitation relates to the relatively small numbers of cases available for retrospective subset analysis, and this might have limited the ability of this study to distinguish real differences between DE and DR imaging. In addition, although the study was designed as a prospective trial, the stratification was performed post hoc and therefore was retrospective.

Overall (all cases and readers pooled), DE imaging performance was equal to DR at equivalent radiation dose; Although DE imaging requires the assessment of a greater number of images compared to DR imaging, this will be compensated for by an increased level of diagnostic confidence in lesion detection, which in turn should translate into the earlier detection of disease. In addition, the characteristics of DE imaging offer promise in other areas of thoracic disease, such as the earlier detection of airspace disease (pneumonia in patients with fever of unknown origin), the improved demonstration of airway disease (bronchiectasis in patients with chronic productive cough), and the improved visualization of catheters, tubes, and pneumothoraces in patients in intensive care units. (69)

The clinical role of DE is yet to be fully understood. The lateral view, for example, is an important aspect of diagnostic chest imaging (e.g., to visualize retrohepatic and retrocardiac lung), but the performance of DE imaging in the lateral view remains to be evaluated. Similarly, the equivalence of the DE "composite" image and a DR image obtained at equivalent total dose is yet to be established. Clinically, high-performance DE imaging at dose equivalent to DR may yield a new normal means of chest radiography, but it does not completely resolve the lack of sensitivity exhibited by DR as a screening modality; rather, the value of DE imaging is likely to be a better first read. Finally, the performance and clinical role of DE imaging with respect to low-dose CT (LDCT) is yet to be fully assessed. While not intended in this work as a 
replacement to LDCT, the use of DE imaging as an adjuvant examination that could help resolve the lack of LDCT specificity is a potentially promising avenue to be investigated.

As an extension of this work, the application of DE imaging in portable radiography is currently under investigation. While maintaining the positive characteristics of DR - i.e., low cost and accessibility - DE imaging could potentially improve the image quality in portable exams. The removal of anatomical noise has even greater importance in this clinical situation where there are often limitations in lesion detectability due to extremely poor image quality in existing portable imaging technologies. Such situations include patients that are unable to be transferred to the radiology department, are obtunded, often examined in a sub-optimal position (supine or rotated), and exhibit limited inspiratory effort, causing under inflation of the lungs. In this study, thoracic DE imaging, performed at equivalent radiation dose to DR, demonstrated superior performance for the detection of small lung nodules that are usually challenging to detect, and the right upper lobe is a common area for missed lung cancers. (68) DE imaging also demonstrated a clinically significant advantage (i.e., a correct change in the diagnostic trajectory of positive cases) as well as more definitive diagnosis of both positive and negative cases. 


\section{References}

1. Taveras JM, Ferrucci JT. "Radiology. Diagnosis-Imaging-Intervention" J.B. Lipincott Co., Philadelphia. 1986

2. Bergin C, Roggli V, Coblentz C, Chiles C. "The secondary pulmonary lobule: normal and abnormal CT appearances" American Journal of Roentgenology.1988; 151(1):21-25.

3. Osborne DR, Effmann EL, Hedlund LW. "Postnatal growth and size of the pulmonary acinus and secondary lobule in man" AJR Am J Roentgenol. 1983; 140(3):449-54.

4. School of anatomy and human biology-the University of Western Australia, Blue histology, respiratory system.

http://www.lab.anhb.uwa.edu.au/mb140/CorePages/Respiratory/respir.htm, accessed : Mar 2010.

5. Angus JE, Thurlbeck WM. "Number of alveoli in the human lung" Appl Physiol. 1972; 32: $483-485$.

6. Koyama S, Sato, E Nomura H, Kubo K, Miura M, Yamashita T, Nagai S, IzumiT.

“Bradykinin.Stimulates Type II Alveolar Cells to Release Neutrophil and Monocyte Chemotactic Activity and Inflammatory Cytokines" American Journal of Pathology. 1998; 153:1885-1893.

7. http://www.who.int/en/accessed : Apr 2010.

8. www.clevelandclinicmeded.com accessed: May 2010.

9. Canadian Cancer statistic, 2009, special topics, cancer in adolescents and young adults. www.Cancer.ca accessed: Sep 2010.

10. Canadian cancer society. http://www.cancer.ca, accessed: Sep 2010.

11. Health Canada, www.hc-sc.gc.ca accessed: Sep 2010. 
12. Alberg AJ, Samet JM. "Epidemiology of lung cancer Chest" 2003; 123(1 Suppl):21S49S.

13. Travis WD. "Pathology of lung cancer" Clin Chest Med. 2002; 23(1):65-81.

14. Takkouche B JesusJ. "The epidemiology of lung cancer: Review of risk factors and Spanish data" Gestal-Otero European Journal of Epidemiology. 1996; 12 (4): 341-349.

15. www.merckaandcoinc.net/mmpe/index.html accessed: Mar 2010.

16. Henschke CI. "International early lung cancer action program: MTP1-01" Journal of Thoracic Oncology. 2007; 2 (8): S267-S268.

17. Swensen SJ, Jett JR, Hartman TE, Midthun DE, Sloan JA, Sykes AM, Aughenbaugh GL, Clemens MA. "Lung cancer screening with CT: The Mayo Clinic experience" Radiology. 2003; 226(3):756-61.

18. Stanbrook MB, Flegel K. "A pause for thought on lung cancer screening" CMAJ. 2009; 180 (8).

19. Strauss GM, Gleason RE, Sugarbaker DJ. "Screening for Lung Cancer, Another look; A Different View" Chest. 1997; 111(3):754-68.

20. National Lung Screening Trial Research Team, Aberle DR, Berg CD, Black WC, Church TR, Fagerstrom RM, Galen B, Gareen IF, et al. "The National Lung Screening Trial: overview and study design" Radiology. 2011; 258(1):243-53.

21. http://www.cancer.gov/newscenter/pressreleases/NLSTresultsRelease. accessed: Jan 2011.

22. Kumar, V. "Robbins Basic Pathology" $7^{\text {th }}$ edition. Elsevier Science. 2005.

23. Mountain CF. "Revisions in the international system for staging lung cancer" Chest. 1997; 111: 1710-17. 
24. Goldstraw P. "The 7th Edition of TNM in Lung Cancer: What Now?" Journal of Thoracic Oncology. 2009; 4 (6): 671-673 .

25. Huq S. "Lung cancer, Non small cell” emedicine, Updated: Sep 15, 2010.

26. KidoS, Kuriyama K, Kuroda C, Nakamura H, Ito W, Shimura K, Kato H. "Detection of simulated pulmonary nodules by single-exposure dual-energy computed radiography of the chest: effect of a computer-aided diagnosis system (Part 2)" Eur J Radiol. 2002; 44(3):205-9.

27. Samei E, Flynn MJ. "An experimental comparison of detector performance for direct and indirect digital radiography systems” Med Phys. 2003; 30:608-622.

28. Antonuk LE, El-Mohri Y, Siewerdsen JH, et al. "Empirical investigation of the signal performance of a high-resolution, indirect detection, active matrix flat-panel imager (AMFPI) for fluoroscopic and radiographic operation" Med Phys. 1997; 24:51-70.

29. Zhao W, Blevis I, Germann S, et al. "Digital radiology using active matrix readout of amorphous selenium: construction and evaluation of a prototype real-time detector" Med Phys. 1997; 24:1834-1843.

30. Austin JHM, Romney BM, Goldsmith LS. "Missed bronchogenic carcinoma: radiographic findings in 27 patients with a potentially resectable lesion evident in retrospect" Radiology. 1992; 182:115-122.

31. Muhm JR, Miller WE, Fontana RS, et al. Lung cancer detected during a screening program using 4-month chest radiographs. Radiology. 1983; 148:609-615.

32. Diederich S, Wormanns D, Semik M, Thomas M, Lenzen H, Roos N, Heindel W. "Screening for early lung cancer with low-dose spiral CT: prevalence in 817 asymptomatic smokers" Radiology. 2002; 222(3):773-8.

33. http://www.hps.org/documents/meddiagimaging.pdf accessed: Sep 2010. 
34. Swensen SJ, Jett JR, Sloan JA, Midthun DE, Hartman TE, Sykes A-M, Aughenbaugh GL, Zink FE, Hillman SL, Noetzel GR, et al . "Screening for lung cancer with low-dose spiral computed tomography” Am J Respir Crit Care Med. 2002; 165: 508-513.

35. Silverman JD, Paul NS, Siewerdsen JH. "Investigation of lung nodule detectability in lowdose 320-slice computed tomography" Med Phys. 2009; 36 (5):1700-10.

36. Daoud W. "Imaging of Lung Disease" Palestinian Medical Journal. 2006; 1 (3): 27-34.

37. Brix G, Lechel U, Glatting G, Ziegler SI, Münzing W, Müller SP, Beyer T. "Radiation Exposure of Patients Undergoing Whole-Body Dual-Modality 18F-FDG PET/CT Examinations" Journal of Nuclear Medicine . 2005; 46(4): 608-613.

38. Behzadi A, Ung Y, LoweV, Deschamps C. "The role of positron emission tomography in the management of non-small cell lung cancer" Can J Surg. 2009; 52( 3): 235-42.

39. Brody WR, Butt G, Hall A, Macovski A. "A method for selective tissue and bone visualization using dual energy scanned projection radiography" Med Phys. 1981; 8:353-357.

40. Burk, Jr. RJ. "Radiation risk in perspective,Position statement of the health physics society " Health Physics Society. 2004.

41. Mitelman F, Johansson B, Mertens FE. Mitelman databaseof chromosome aberations in cancer. Cancer Genome Anatomy Project, http://cgap.nci.nih.gov/. 2007; accessed Jan 2011.

42. Berenner DJ, Hall EJ. " Computed tomography- an increasing source of radiation exposure “ N Engl J Med. 2007; 357(22):2277-84

43. Smith-Bindman $\mathrm{R}$ et al. "Radiation dose associated with common computed tomography examinations and the associated lifetime attributable risk of cancer" Arch Intern Med. 2009; 169(22):2078-86.

44. Shkumat NA, Siewerdsen JH, Dhanantwari AC, et al. "Cardiac gating with a pulse oximeter for dual-energy imaging" Med Phys. 2008; 53: 6097-6112. 
45. Siewerdsen JH, Shkumat NA, Dhanantwari AC, Williams DB, Richard S, Daly MJ, Paul NS, Moseley DJ, Jaffray DA, Yorkston J, Van Metter R. "High-Performance Dual-Energy Imaging With a Flat-Panel Detector: Imaging Physics From Blackboard to Benchtop to Bedside" Proc.SPIE Physics of Medical Imaging. 2006; 6142: 61421E-1-61421E-10.

46. Shkumat NA, Siewerdsen JH, Dhanantwari AC, et al. "Optimization of image acquisition techniques for dual-energy imaging of the chest" Med Phys. 2007; 34:3904-3915.

47. Shkumat NA, Siewerdsen JH, Richard S, et al. "Dual-energy imaging of the chest: optimization of image acquisition techniques for the bone only image" Med Phys. 2008; 35:629-632.

48. Richard S, Siewerdsen JH. "Cascaded systems analysis of noise reduction algorithms in dual-energy imaging" Med Phys. 2008; 35:586-601.

49. Dhanantwari A, Siewerdsen J, Shkumat N, et al. "Multi-resolution, multiscale mutual information technique for registration of a high- and low-kVp projections in dual-energy imaging" Med Phys. 2007; 34:2517.

50. Kalender WA, Klotz E, Kostaridou L. "An algorithm for noise suppression in dual energy CT material density images” IEEE Trans Med Imaging. 1988; 7:218-224.

51. McCollough $\mathrm{CH}$, Van Lysel MS, Peppler WW, et al. "A correlated noise reduction algorithm for dual-energy digital subtraction angiography" Med Phys. 1989; 16:873-880.

52. Johns PC, Yaffe MJ. "Theoretical optimization of dual-energy x-ray imaging with application to mammography" Med Phys. 1985; 12:289-296.

53. Kashani H, Gang JG, Shkumat NA, Varon CA, Yorkston J, Van Metter R, Paul NS, Siewerdsen $\mathrm{JH}$. "Development of a high-performance dual-energy chest imaging system: initial investigation of diagnostic performance" Acad Radiol. 2009;16(4):464-76.

54. Gohari A, Haramati LB. "Complication of CT scan-guided lung biopsy" Chest. 2004; 126:666-668. 
55. Kashani H, Varon CA, Paul NS, Gang GJ, Van Metter R, Yorkston J, Siewerdsen JH. "Diagnostic performance of a prototype dual-energy chest imaging system ROC analysis" Acad Radiol. 2010; 17(3):298-308.

56. Lee BW, Wain JC, Kelsey KT, Wiencke JK, Christiani DC. "Association between diet and lung cancer location" Am J Respir Crit Care Med. 1998; 158(4): 1197-203.

57. Gang GJ, Varon CA, Kashani H, Richard S, Paul NS, Van Metter R, Yorkston J, Siewerdsen $\mathrm{JH}$. "Multiscale deformable registration for dual-energy x-ray imaging" Med Phys. 2009: 36(2): 351-63.

58. Van Metter R, Foos D. "Enhanced latitude for digital projection radiography" Proc SPIE Conf Image Display. 1999; 3658:468-483.

59. Barski LL, Van Metter R, Foos DH, Lee HC, Wang X. "New automatic tone scale method for computed radiography" Proc. SPIE. 1998; 3335:164-178.

60. Rosner B, Glynn R, Lee MT. "The Wilcoxon signed rank test for paired comparisons of clustered data" Biometrics. 2006; 62:185-192.

61. Abdi, H. "Bonferroni and Šidák corrections for multiple comparisons" Encyclopedia of Measurement and Statistics. 2007; Thousand Oaks, CA.

62. Obuchowski NA. “ROC Analysis” AJR Am J Roentgenol. 2005; 184:364-372.

63. Rockette HE, Gur D. "Selection of a rating scale in receiver operating characteristic studies: some remaining issues" Acad Radiol. 2008; 15(2):245-8.

64. Hadjiiski L, Chan HP, Sahiner B, Helvie MA, Roubidoux MA. "Quasi-continuous and discrete confidence rating scales for observer performance studies: Effects on ROC analysis" .Acad Radiol. 2007; 14(1): 38-48. 
65. Dorfman DD, ALP E. "Maximum likelihood estimation of parameters of signal detection theory-A direct solution" Psychometrika. 1968; 33 (1): 117-124.

66. Metz CE, Pan X. " Proper' Binormal ROC Curves: Theory and Maximum-Likelihood Estimation" Journal of Mathematical Psychology. 1999; 43 (1):1-33.

67. DeLong ER, DeLong DM, Clarke-Pearson DL. "Comparing the Areas Under Two or More Correlated Receiver Operating Characteristic Curves: A Nonparametric Approach" BIOMETRICS. 1988; 44: 837-845.

68. Shah PK, Austin JH, White CS, et al. "Missed non-small cell lung cancer: radiographic findings of potentially resectable lesions evident only in retrospect" Radiology. 2003; 226:235-241.

69. Kuhlman JE, Collins J, Brooks GN, Yandow DR, Broderick LS. "Dual-energy subtraction chest radiography: what to look for beyond calcified nodules" Radiographics. 2006; 26:79-92. 


\title{
Copyright Acknowledgements
}

\author{
ELSEVIER LICENSE \\ TERMS AND CONDITIONS
}

Aug 10, 2010

This is a License Agreement between Hany Kashani ("You") and Elsevier ("Elsevier") provided by Copyright Clearance Center ("CCC"). The license consists of your order details, the terms and conditions provided by Elsevier, and the payment terms and conditions.

All payments must be made in full to CCC. For payment instructions, please see information listed at the bottom of this form.

\begin{tabular}{|c|c|}
\hline \multirow[t]{3}{*}{ Supplier } & Elsevier Limited \\
\hline & The Boulevard,Langford Lane \\
\hline & Kidlington,Oxford,OX5 1GB,UK \\
\hline Registered Company & 1982084 \\
\hline \multicolumn{2}{|l|}{ Number } \\
\hline Customer name & hany Kashani \\
\hline Customer address & 208 Queens Quay West \#3211, Toronto, ON M5J2Y5 \\
\hline License number & 2406591375800 \\
\hline License date & Apr 12, 2010 \\
\hline publisher & E Isevier \\
\hline publication & Academic Radiology \\
\hline \multirow[t]{2}{*}{ Licensed content title } & Development of a High-performance Dual-energy Chest Imaging \\
\hline & System: Initial Investigation of Diagnostic Performance \\
\hline \multirow[t]{3}{*}{ Licensed content author } & Hany Kashani, Jianan Grace Gang, Nicholas A. Shkumat, \\
\hline & Carlos A. Varon, John Yorkston, Richard Van Metter, Narinder \\
\hline & S. Paul, Jeffrey H. Siewerdsen \\
\hline
\end{tabular}




\begin{tabular}{|c|c|}
\hline Licensed content date & April 2009 \\
\hline $\begin{array}{l}\text { Licensed content volume } \\
\text { number }\end{array}$ & 16 \\
\hline $\begin{array}{l}\text { Licensed content issue } \\
\text { number }\end{array}$ & 4 \\
\hline Number of pages & 13 \\
\hline Type of Use & Thesis / Dissertation \\
\hline Requestor type & NOTSELECTED \\
\hline $\begin{array}{l}\text { Intended publisher of } \\
\text { new work }\end{array}$ & NOTSELECTED \\
\hline Other institution name & NOTSELECTED \\
\hline Portion & Full article \\
\hline Format & Both print and electronic \\
\hline Are you the author of this & Yes \\
\hline Elsevier article? & \\
\hline Order reference number & NOT SELECTED \\
\hline Elsevier VAT number & GB 494627212 \\
\hline
\end{tabular}

Terms and Conditions INTRODUCTION

1. The publisher for this copyrighted material is Elsevier. By clicking "accept" in connection with completing this licensing transaction, you agree that the following terms and conditions apply to this transaction (along with the Billing and Payment terms and conditions established by Copyright Clearance Center, Inc. ("CCC"), at the time that you opened your Rightslink account and that are available at any time at http://myaccount.copyright.com).

\section{GENERAL TERMS}


2. Elsevier hereby grants you permission to reproduce the aforementioned material subject to the terms and conditions indicated.

3. Acknowledgement: If any part of the material to be used (for example, figures) has appeared in our publication with credit or acknowledgement to another source, permission

must also be sought from that source. If such permission is not obtained then that material may not be included in your publication/copies. Suitable acknowledgement to the source must be made, either as a footnote or in a reference list at the end of your publication, as follows:

"Reprinted from Publication title, Vol /edition number, Author(s), Title of article / title of chapter, Pages No., Copyright (Year), with permission from Elsevier [OR APPLICABLE SOCIETY COPYRIGHT OWNER]." Also Lancet special credit - "Reprinted from The Lancet, Vol. number, Author(s), Title of article, Pages No., Copyright (Year), with permission from Elsevier."

4. Reproduction of this material is confined to the purpose and/or media for which permission is hereby given.

5. Altering/Modifying Material: Not Permitted. However figures and illustrations may be altered/adapted minimally to serve your work. Any other abbreviations, additions, deletions and/or any other alterations shall be made only with prior written authorization of Elsevier Ltd. (Please contact Elsevier at permissions@elsevier.com)

6. If the permission fee for the requested use of our material is waived in this instance, please be advised that your future requests for Elsevier materials may attract a fee.

7. Reservation of Rights: Publisher reserves all rights not specifically granted in the combination of (i) the license details provided by you and accepted in the course of this licensing transaction, (ii) these terms and conditions and (iii) CCC's Billing and Payment terms and conditions.

8. License Contingent Upon Payment: While you may exercise the rights licensed immediately upon issuance of the license at the end of the licensing process for the transaction, provided that you have disclosed complete and accurate details of your proposed use, no license is finally effective unless and until full payment is received from you (either by publisher or by CCC) as provided in CCC's Billing and Payment terms and conditions. If full payment is not received on a timely basis, then any license preliminarily granted shall be deemed automatically revoked and shall be void as if never granted. Further, in the event. 
that you breach any of these terms and conditions or any of CCC's Billing and Payment terms and conditions, the license is automatically revoked and shall be void as if never granted. Use of materials as described in a revoked license, as well as any use of the materials beyond the scope of an unrevoked license, may constitute copyright infringement and publisher reserves the right to take any and all action to protect its copyright in the materials.

9. Warranties: Publisher makes no representations or warranties with respect to the licensed material.

10. Indemnity: You hereby indemnify and agree to hold harmless publisher and CCC, and their respective officers, directors, employees and agents, from and against any and all claims arising out of your use of the licensed material other than as specifically authorized pursuant to this license.

11. No Transfer of License: This license is personal to you and may not be sublicensed, assigned, or transferred by you to any other person without publisher's written permission.

12. No Amendment Except in Writing: This license may not be amended except in a writing signed by both parties (or, in the case of publisher, by CCC on publisher's behalf).

13. Objection to Contrary Terms: Publisher hereby objects to any terms contained in any purchase order, acknowledgment, check endorsement or other writing prepared by you, which terms are inconsistent with these terms and conditions or CCC's Billing and Payment terms and conditions. These terms and conditions, together with CCC's Billing and Payment terms and conditions (which are incorporated herein), comprise the entire agreement between you and publisher (and CCC) concerning this licensing transaction. In the event of any conflict between your obligations established by these terms and conditions and those established by CCC's Billing and Payment terms and conditions, these terms and conditions shall control.

14. Revocation: Elsevier or Copyright Clearance Center may deny the permissions described in this License at their sole discretion, for any reason or no reason, with a full refund payable to you. Notice of such denial will be made using the contact information provided by you

Failure to receive such notice will not alter or invalidate the denial. In no event will Elsevier or Copyright Clearance Center be responsible or liable for any costs, expenses or damage incurred by you as a result of a denial of your permission request, other than a refund of the amount(s) paid by you to Elsevier and/or Copyright Clearance Center for denied permissions. 


\section{LIMITED LICENSE}

The following terms and conditions apply only to specific license types:

15. Translation: This permission is granted for non-exclusive world English rights only unless your license was granted for translation rights. If you licensed translation rights you may only translate this content into the languages you requested. A professional translator must perform all translations and reproduce the content word for word preserving the integrity of the article. If this license is to re-use 1 or 2 figures then permission is granted for non-exclusive world rights in all languages.

16. Website: The following terms and conditions apply to electronic reserve and author websites:

Electronic reserve: If licensed material is to be posted to website, the web site is to be password-protected and made available only to bona fide students registered on a relevant course if:

This license was made in connection with a course,

This permission is granted for 1 year only. You may obtain a license for future website posting, All content posted to the web site must maintain the copyright information line on the bottom of each image,

A hyper-text must be included to the Homepage of the journal from which you are licensing at http://www.sciencedirect.com/science/journal/xxxxx or the Elsevier homepage for books at http://www.elsevier.com , and

Central Storage: This license does not include permission for a scanned version of the material to be stored in a central repository such as that provided by Heron/XanEdu.

17. Author website for journals with the following additional clauses:

All content posted to the web site must maintain the copyright information line on the bottom of each image, and

he permission granted is limited to the personal version of your paper. You are not allowed to download and post the published electronic version of your article (whether PDF or HTML, proof or final version), nor may you scan the printed edition to create an electronic version, A hyper-text must be included to the Homepage of the journal from which you are licensing at http://www.sciencedirect.com/science/journal/xxxxx , As part of our normal production process, you will receive an e-mail notice when your article appears on Elsevier's online service ScienceDirect (www.sciencedirect.com). That e-mail will include the article's Digital Object 
Identifier (DOI). This number provides the electronic link to the published article and should be included in the posting of your personal version. We ask that you wait until you receive this email and have the DOI to do any posting.

Central Storage: This license does not include permission for a scanned version of the material to be stored in a central repository such as that provided by Heron/XanEdu.

18. Author website for books with the following additional clauses:

Authors are permitted to place a brief summary of their work online only.

A hyper-text must be included to the Elsevier homepage at http://www.elsevier.com

All content posted to the web site must maintain the copyright information line on the bottom of each image

You are not allowed to download and post the published electronic version of your chapter, nor may you scan the printed edition to create an electronic version.

Central Storage: This license does not include permission for a scanned version of the material to be stored in a central repository such as that provided by Heron/XanEdu.

19. Website (regular and for author): A hyper-text must be included to the Homepage of the journal from which you are licensing at

http://www.sciencedirect.com/science/journal/xxxxx. Or for books to the Elsevier homepage at http://www.elsevier.com

20. Thesis/Dissertation: If your license is for use in a thesis/dissertation your thesis may be submitted to your institution in either print or electronic form. Should your thesis be published commercially, please reapply for permission. These requirements include permission for the Library and Archives of Canada to supply single copies, on demand, of the complete thesis and include permission for UMI to supply single copies, on demand, of the complete thesis. Should your thesis be published commercially, please reapply for permission.

\section{Other Conditions: None}




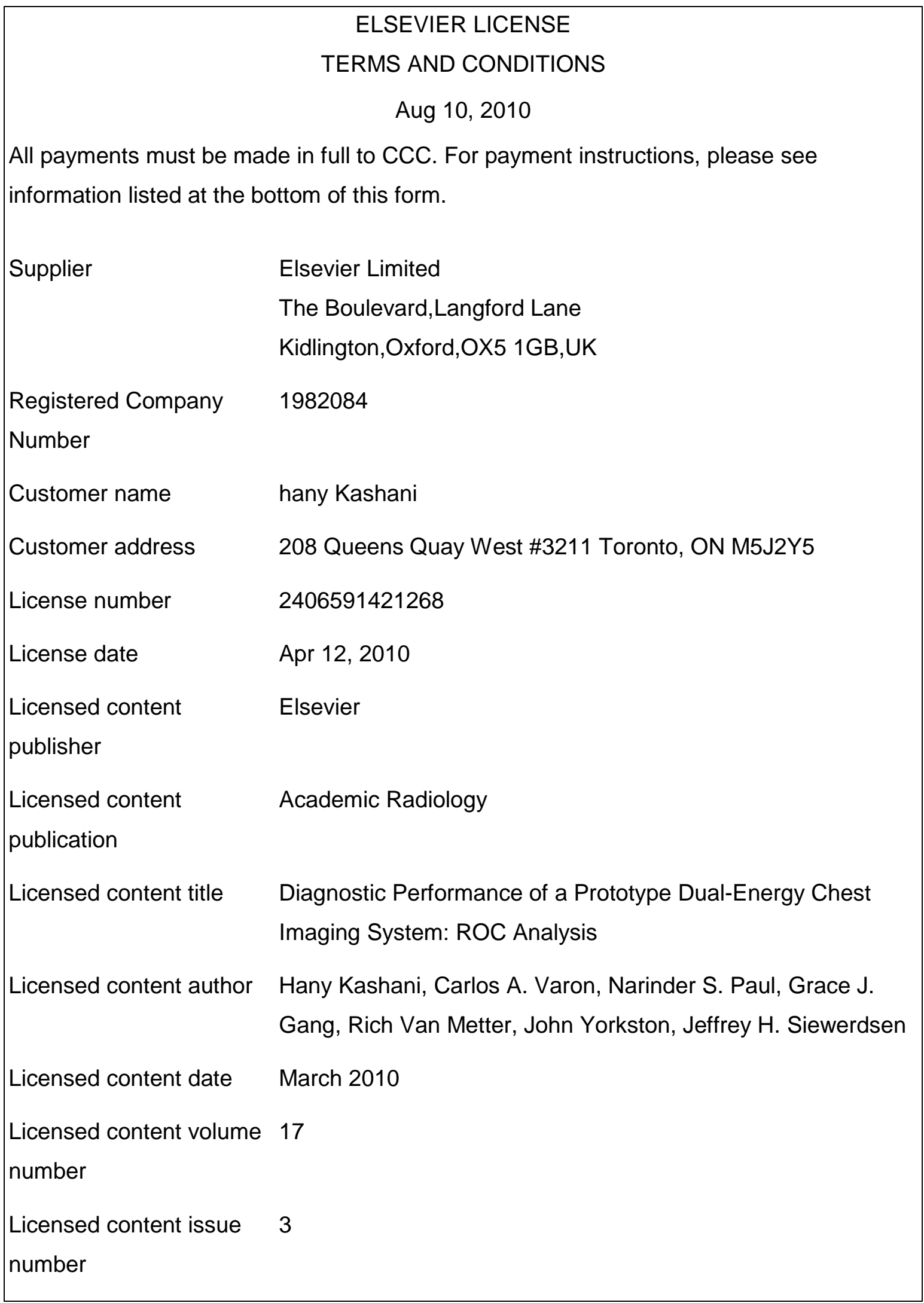




\begin{tabular}{|c|c|}
\hline Number of pages & 11 \\
\hline Type of Use & Thesis / Dissertation \\
\hline Requestor type & NOTSELECTED \\
\hline Intended publisher of new & NOTSELECTED \\
\hline work & \\
\hline Other institution name & NOTSELECTED \\
\hline Portion & Full article \\
\hline Format & Both print and electronic \\
\hline Are you the author of this & Yes \\
\hline Elsevier article? & \\
\hline Order reference number & NOT SELECTED \\
\hline Elsevier VAT number & GB 494627212 \\
\hline
\end{tabular}

\section{Terms and Conditions}

\section{INTRODUCTION}

1. The publisher for this copyrighted material is Elsevier. By clicking "accept" in connection with completing this licensing transaction, you agree that the following terms and conditions apply to this transaction (along with the Billing and Payment terms and conditions established by Copyright Clearance Center, Inc. ("CCC"), at the time that you opened your Rightslink account and that are available at any time at http://myaccount.copyright.com).

\section{GENERAL TERMS}

2. Elsevier hereby grants you permission to reproduce the aforementioned material subject to the terms and conditions indicated.

3. Acknowledgement: If any part of the material to be used (for example, figures) has appeared in our publication with credit or acknowledgement to another source, permission must also be sought from that source. If such permission is not obtained then that material may not be included in your publication/copies. Suitable acknowledgement to the source must be made, either as a footnote or in a reference list at the end of your publication, as follows: 
"Reprinted from Publication title, Vol /edition number, Author(s), Title of article / title of chapter, Pages No., Copyright (Year), with permission from Elsevier [OR APPLICABLE SOCIETY COPYRIGHT OWNER]." Also Lancet special credit - "Reprinted from The Lancet, Vol. number, Author(s), Title of article, Pages No., Copyright (Year), with permission from Elsevier."

4. Reproduction of this material is confined to the purpose and/or media for which permission is hereby given.

5. Altering/Modifying Material: Not Permitted. However figures and illustrations may be altered/adapted minimally to serve your work. Any other abbreviations, additions, deletions and/or any other alterations shall be made only with prior written authorization of Elsevier Ltd. (Please contact Elsevier at permissions@elsevier.com)

6. If the permission fee for the requested use of our material is waived in this instance, please be advised that your future requests for Elsevier materials may attract a fee.

7. Reservation of Rights: Publisher reserves all rights not specifically granted in the combination of (i) the license details provided by you and accepted in the course of this licensing transaction, (ii) these terms and conditions and (iii) CCC's Billing and Payment terms and conditions.

8. License Contingent Upon Payment: While you may exercise the rights licensed immediately upon issuance of the license at the end of the licensing process for the transaction, provided that you have disclosed complete and accurate details of your proposed use, no license is finally effective unless and until full payment is received from you (either by publisher or by CCC) as provided in CCC's Billing and Payment terms and conditions. If full payment is not received on a timely basis, then any license preliminarily granted shall be deemed automatically revoked and shall be void as if never granted. Further, in the event that you breach any of these terms and conditions or any of CCC's Billing and Payment terms and conditions, the license is automatically revoked and shall be void as if never granted. Use of materials as described in a revoked license, as well as any use of the materials beyond the scope of an unrevoked license, may constitute copyright infringement and publisher reserves the right to take any and all action to protect its copyright in the materials.

9. Warranties: Publisher makes no representations or warranties with respect to the licensed material. 
10. Indemnity: You hereby indemnify and agree to hold harmless publisher and CCC, and their respective officers, directors, employees and agents, from and against any and all claims arising out of your use of the licensed material other than as specifically authorized pursuant to this license.

11. No Transfer of License: This license is personal to you and may not be sublicensed, assigned, or transferred by you to any other person without publisher's written permission.

12. No Amendment Except in Writing: This license may not be amended except in a writing signed by both parties (or, in the case of publisher, by CCC on publisher's behalf).

13. Objection to Contrary Terms: Publisher hereby objects to any terms contained in any purchase order, acknowledgment, check endorsement or other writing prepared by you, which terms are inconsistent with these terms and conditions or CCC's Billing and Payment terms and conditions. These terms and conditions, together with CCC's Billing and Payment terms and conditions (which are incorporated herein), comprise the entire agreement between you and publisher (and CCC) concerning this licensing transaction. In the event of any conflict between your obligations established by these terms and conditions and those established by CCC's Billing and Payment terms and conditions, these terms and conditions shall control.

14. Revocation: Elsevier or Copyright Clearance Center may deny the permissions described in this License at their sole discretion, for any reason or no reason, with a full refund payable to you. Notice of such denial will be made using the contact information provided by you. Failure to receive such notice will not alter or invalidate the denial. In no event will Elsevier or Copyright Clearance Center be responsible or liable for any costs, expenses or damage incurred by you as a result of a denial of your permission request, other than a refund of the amount(s) paid by you to Elsevier and/or Copyright Clearance Center for denied permissions.

\section{LIMITED LICENSE}

The following terms and conditions apply only to specific license types:

15. Translation: This permission is granted for non-exclusive world English rights only unless your license was granted for translation rights. If you licensed translation rights you may only translate this content into the languages you requested. A professional translator must perform all translations and reproduce the content word for word preserving the integrity of the article. If 
this license is to re-use 1 or 2 figures then permission is granted for non-exclusive world rights in all languages.

16. Website: The following terms and conditions apply to electronic reserve and author websites:

Electronic reserve: If licensed material is to be posted to website, the web site is to be password-protected and made available only to bona fide students registered on a relevant course if:

This license was made in connection with a course,

This permission is granted for 1 year only. You may obtain a license for future website posting, All content posted to the web site must maintain the copyright information line on the bottom of each image,

A hyper-text must be included to the Homepage of the journal from which you are licensing at http://www.sciencedirect.com/science/journal/xxxxx or the Elsevier homepage for books at http://www.elsevier.com , and

Central Storage: This license does not include permission for a scanned version of the material to be stored in a central repository such as that provided by Heron/XanEdu.

17. Author website for journals with the following additional clauses:

All content posted to the web site must maintain the copyright information line on the bottom of each image, and

he permission granted is limited to the personal version of your paper. You are not allowed to download and post the published electronic version of your article (whether PDF or HTML, proof or final version), nor may you scan the printed edition to create an electronic version, A hyper-text must be included to the Homepage of the journal from which you are licensing at http://www.sciencedirect.com/science/journal/xxxxx , As part of our normal production process, you will receive an e-mail notice when your article appears on Elsevier's online service ScienceDirect (www.sciencedirect.com). That e-mail will include the article's Digital Object Identifier (DOI). This number provides the electronic link to the published article and should be included in the posting of your personal version. We ask that you wait until you receive this email and have the DOI to do any posting.

Central Storage: This license does not include permission for a scanned version of the material to be stored in a central repository such as that provided by Heron/XanEdu. 
18. Author website for books with the following additional clauses:

Authors are permitted to place a brief summary of their work online only.

A hyper-text must be included to the Elsevier homepage at http://www.elsevier.com

All content posted to the web site must maintain the copyright information line on the bottom of each image

You are not allowed to download and post the published electronic version of your chapter, nor may you scan the printed edition to create an electronic version.

Central Storage: This license does not include permission for a scanned version of the material to be stored in a central repository such as that provided by Heron/XanEdu.

19. Website (regular and for author): A hyper-text must be included to the Homepage of the journal from which you are licensing at http://www.sciencedirect.com/science/journal/xxxxx. or for books to the Elsevier homepage at http://www.elsevier.com

20. Thesis/Dissertation: If your license is for use in a thesis/dissertation your thesis may be submitted to your institution in either print or electronic form. Should your thesis be published commercially, please reapply for permission. These requirements include permission for the Library and Archives of Canada to supply single copies, on demand, of the complete thesis and include permission for UMI to supply single copies, on demand, of the complete thesis. Should your thesis be published commercially, please reapply for permission.

21. Other Conditions: None 